
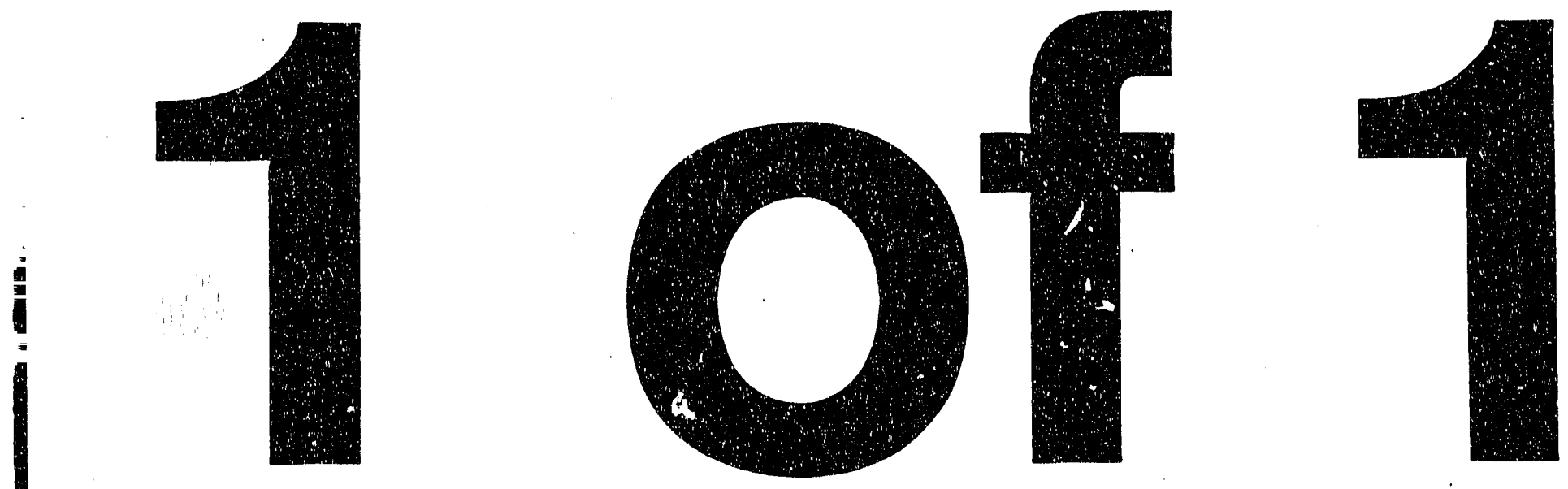


\section{Development of Environmentally Conscious Cleaning Process for Leadless Chip Carrier Assemblies}

Kansas City Division

B. E. Adams

KCP-613-5556

Published April 1995

Final Report

Approved for public release; distribution is unlimited.

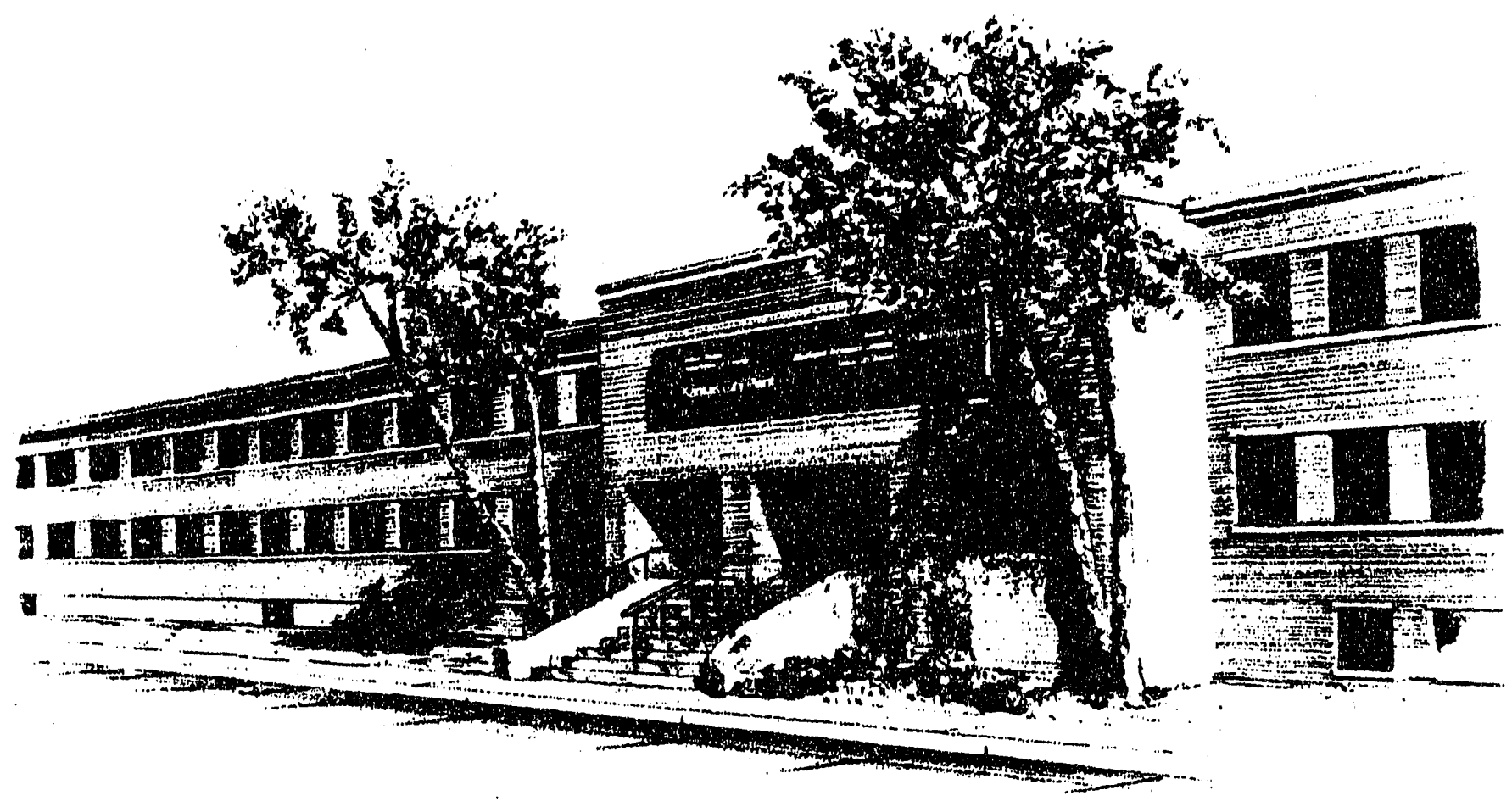

Prepared Under Contract Number DE-ACO4-76-DP00613 for the United States Department of Energy

AlliedSignal

A E R O S P A C E 


\section{DISCLAIMER}

This report was prepared as an account of work sponsored by an agency of the United States Government. Neither the United States Government nor any agency thereof, nor any of their employees, makes any warranty, express or implied, or assumes any legal liability or responsibility for the accuracy, completeness, or usefulness of any information, apparatus, product, or process disclosed, or represents that its use would not infringe privately owned rights. Reference herein to any specific commercial product, process, or service by trade names, trademark, manufacturer, or otherwise, does not necessarily constitute or imply its endorsement, recommendation, or favoring by the United States Government or any agency thereof. The views and opinions of authors expressed herein do not necessarily state or reflect those of the United States Government or any agency thereof.

Printed in the United States of America.

This report has been reproduced from the best available copy.

Available to DOE and DOE contractors from the Office of Scientific and Technical Information, P. O. Box 62, Oak Ridge, Tennessee 37831; prices available from (615) 576-8401, FTS 626-8401.

Available to the public from the National Technical Information Service, U. S. Department of Commerce, 5285 Port Royal Rd., Springfield, Virginia 22161. 
KCP-613-5556

Distribution Category UC-706

Approved for public release; distribution is unlimited.

\title{
DEVELOPMENT OF ENVIRONMENTALLY CONSCIOUS CLEANING PROCESS FOR LEADLESS CHIP CARRIER ASSEMBLIES
}

\author{
B. E. Adams
}

Published April 1995

Final Report

B. E. Adams, Project Leader

Project Team:

J. L. Beatty

G. W. Bohnert

R. E. Culver

S. Garrett

T. E. Fiand

D. Kramer

C. Yoon

\section{DISCLAIMER}

\begin{abstract}
This report was prepared as an account of work sponsored by an agency of the United States
Governrnent. Neither the United States Government nor any agency thereof, nor any of their
employees, makes any warranty, express or implied, or asumes bility for the accuracy, completeness, or usefulness, or assumes any legal liability or responsiprocess disclosed, or represents that its use would of any information, apparatus, product, or ence herein to any specific commercial product, prot infringe privately owned rights. Refermanufacturer, or otherwise does not necessarily process, or service by trade name, trademark, mendation, or favoring by the United States Government or imply its endorsement, recomand opinions of authors expressed herein Government or any agency thereof. The views United States Government or any agency thereof.
necessarily state or reflect those of the
\end{abstract}




\section{Contents}

$\begin{array}{ll}\text { Section Page } & \text { Pan }\end{array}$

Abstract ............................................................................. 1

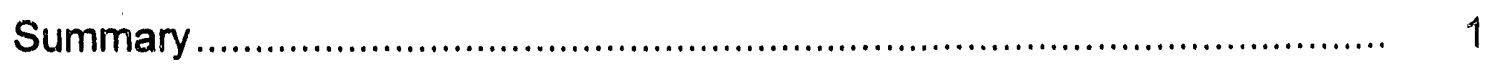

Discussion ........................................................................ 3

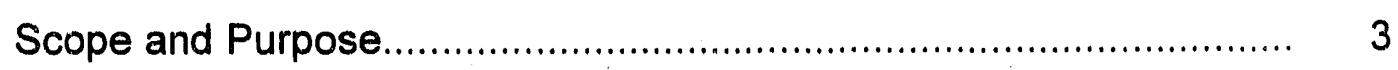

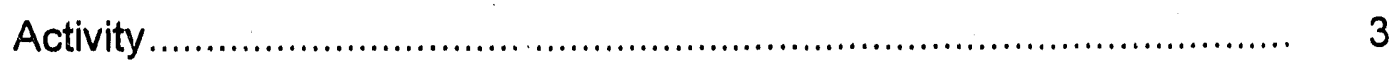

Background ............................................................ 3

Experimentation .................................................... 4

Results .................................................................. 6

Accomplishments .................................................... 9

Appendix. Electrical Test Data for Common Radar LCCAs .................. 11 


\section{Illustrations}

Figure

Page

1 Auger Results for Ceramic Packages ........................................ 7

2 Auger Results for Kovar Lids ............................................. 8

\section{Tables}

Number

Page

1 Yield Data for LCCAs Cleaned by Aqueous and Freon Processes (A) ......... 2

2. Number of Particles Captured During Flush and Filter Testing ................. 6

3 Yield Data for LCCAs Cleaned by Aqueous and Freon Processes (B) ......... 8

4 Internal Moisture Results for Aqueous- and Freon-Cleaned LCCAs

Sealed the Same Day

5 Production Yields at Critical Points for Aqueous- and Freon-Cleaned LCCAs.

6 Internal Moisture Results for Freon- and Aqueous-Cleaned LCCAs After 500-Hour HTRB.

A1 Minimum and Maximum Values of Initial Electrical Test Data for M-53 ........ 12

A2 Average and Standard Deviation of Initial Electrical Test Data for M-53...... 13

A3 Minimum and Maximum Values of Post HTRB Electrical Test Data

for M-53.

A4 Average and Standard Deviation of Post HTRB Electrical Test Data for $M-53$.

A5 Minimum and Maximum Values of Post Extended HTRB Electrical

Test Data for M-53

A6 Average and Standard Deviation of Post Extended HTRB Electrical

Test Data for M-53 
A7 Minimum and Maximum Values of Post 500 Hour HTRB Electrical

Test Data for M-53

A8 Average and Standard Deviation of Post 500 Hour HTRB Electrical

Test Data for M-53

A9 Minimum and Maximum Values of Electrical Test Data at $-55^{\circ} \mathrm{C}$

for $M-53$

A10 Average and Standard Deviation of Electrical Test Data at $-55^{\circ} \mathrm{C}$ for $M-53$

A11 Minimum and Maximum Values of Electrical Test Data at $100^{\circ} \mathrm{C}$ for $M-53$

A12 Average and Standard Deviation of Electrical Test Data at $100^{\circ} \mathrm{C}$ for $M-53$

A13 Minimum and Maximum Values of Initial Electrical Test Data for M-48 ....... 23

A14 Average and Standard Deviation of Initial Electrical Test Data for M-48 ......

A15 Minimum and Maximum Values of Post HTRB Electrical Test Data for $M-48$

A16 Average and Standard Deviation of Post HTRB Electrical Test Data for $M-48$

A17 Minimum and Maximum Values of Post Extended HTRB Electrical Test Data for $\mathrm{M}-48$

A18 Average and Standard Deviation of Post Extended HTRB Electrical Test Data for $M-48$

A19 Minimum and Maximum Values of Post 500 Hour HTRB Electrical Test Data for $M-48$

A20 Average and Standard Deviation of Post 500 Hour HTRB Electrical Test Data for $\mathrm{M}-48$

A21 Minimum and Maximum Values of Electrical Test Data at $-55^{\circ} \mathrm{C}$ for $M-48$.

A22 Average and Standard Deviation of Electrical Test Data at $-55^{\circ} \mathrm{C}$ for $M-48$.

A23 Minimum and Maximum Values of Electrical Test Data at $100^{\circ} \mathrm{C}$ for $M-48$. 
A24 Average and Standard Deviation of Electrical Test Data at $100^{\circ} \mathrm{C}$ for $M-48$.

A25 Minimum and Maximum Values of Initial Electrical Test Data for M-50...... 29

A26 Average and Standard Deviation of Initial Electrical Test Data for M-50 ..... 31

A27 Minimum and Maximum Values of Post HTRB Electrical Test Data for $M-50$.

A28 Average and Standard Deviation of Post HTRB Electrical Test Data for $M-50$.

A29 Minimum and Maximum Values of Post Extended HTRB Electrical Test Data for M-50

A30 Average and Standard Deviation of Post Extended HTRB Electrical Test Data for M-50

A31 Minimum and Maximum Values of Post 500 Hour HTRB Electrical Test Data for M-50

A32 Average and Standard Deviation of Post 500 Hour HTRB Electrical Test Data for M-50

A33 Minimum and Maximum Values of Electrical Test Data at $-55^{\circ} \mathrm{C}$ for $M-50$.

A34 Average and Standard Deviation of Electrical Test Data at $-55^{\circ} \mathrm{C}$ for $M-50$.

A35 Minimum and Maximum Values of Electrical Test Data at $100^{\circ} \mathrm{C}$ for $M-50$.

A36 Average and Standard Deviation of Electrical Test Data at $100^{\circ} \mathrm{C}$ for M-50. 


\section{Abstract}

A cross-functional team of process, product, quality, material, and design lab engineers was assembled to develop an environmentally friendly cleaning process for leadless chip carrier assemblies (LCCAS). Using flush and filter testing, Auger surface analysis, GC-Mass spectrophotometry, production yield results, and electrical test results over an extended iesting period, the team developed an aqueous cleaning process for LCCAs. The aqueous process replaced the Freon vapor degreasing/ultrasonic rinse process.

\section{Summary}

A cross-functional team of process, product, quality, material, and design lab engineers was assembled to investigate the cleaning issues for leadless chip carrier assemblies (LCCAs). The purpose for this investigation was to develop an environmentally friendly cleaning process for LCCAs to eliminate the use of Freon. Using the TQ process, the team analyzed the current process, brainstormed possible alternatives, and then investigated their feasibility. To identify the root causes of contamination, all storage conditions and handling procedure for all components and assemblies from stores through production were investigated. The major contaminants of concern were ionic, particulate, and human debris.

The testing methods used to determine component and assembly cleanliness included flush and filter testing, Auger surface analysis, and GC-Mass spectrophotometry: Flush and filter testing was used to determine the quantity of particles that were 0.45 microns and larger on a part. The procedure was to ultrasonically clean $(40 \mathrm{KHz})$ a part for 2 minutes. The part was contained in a test tube filled with $20 \mathrm{ml}$ of Dl water filtered to 0.2 micron. The contents of the test tube were then vacuum filtered through 0.45 -micron filter paper. The paper was examined under 640X microscope to look for particles. All work was done in a class 100 clean bench. Several blanks and base line runs were done before and during the testing to determine background particle contamination. Auger surface analysis was run to base line the organic contamination level and measure the cleaning ability of several solvents. GC-Mass spectrophotometry was used to identify the organic contaminants.

The results of the flush and filter testing showed that the most grossly contaminated parts were packages after die attach and the die attach collets. A high number of silicon, gold, ceramic, eutectic, and white foreign material particles were found in the packages and on the collets. No particle contamination was removed from Kovar lids. Auger surface analysis revealed LCC packages were not contaminated by organic materials. Auger of the Kovar lids did reveal organic materials present. Cleaning the lids by alcohol or oxygen plasma removed the organic material. GC-Mass spectrophotometry identified the contaminants as oleamide, erucamide (slip agents used on polyethylene bags), and trace amounts of polyethylene. On the average, $0.01 \mathrm{mg}$ of residue was on each lid. From these results it was determined that LCCAs should be ultrasonically cleaned in DI water before and after die attach to remove particles and ionic. 
A DI water flush in a cascading tank was recommended for assemblies prior to sealing. The small amount of contamination on lids was considered insignificant, and lids were not to be cleaned.

With a new aqueous process developed, verification of product producibility and reliability was required. To measure producibility, sample parts from excess material were assembled and production yields were measured. To measure reliability, six lots of commion radar parts were assembled and tested. Three lots were assembled using WR procedures and cleaned with CFC-113. Three identical lots were assembled following the WR proredures and substituting the new aqueous cleaning process for the CFC-113. The lots were tested to the following requirements.

- The assemblies were tested to the requirements of their respective product specifications.

- Twenty pieces from each lot underwent high temperature reverse bias (HTRB) for 500 hours at $175^{\circ} \mathrm{C}$. No failures could occur when electrically tested.

- Moisture analysis of five of each set of twenty parts was rur. Moisture level must be below $3000 \mathrm{ppm}$.

- Comparison was made between the data collected on aqueous-cleaned and CFC-cleaned parts.

Results of the producibility experiments, in Table 1, showed production yields to be equal for Freon- and aqueous-cleaned parts. The comparison of electrical data between Freon-cleaned LCCAs and aqueous-cleaned LCCAs showed no statistical difference. Residual gas analysis indicated that average moisture in Freon-cleaned LCCAs was 392 ppm and for aqueous cleaned LCCAs was 498. ppm after 500-hour life test.

Table 1. Yield Data for LCCAs Cleaned by Aqueous and Freon Processes

\begin{tabular}{|c|c|c|}
\hline & Aqueous & Freon (Historical) \\
\hline PIND (5 Runs) & $24 / 25(96 \%)$ & $96.4 \%$ \\
\hline NDPT & $150 / 150(100 \%)$ & $99.5 \%$ \\
\hline Sealing & $140 / 140(100 \%)$ & $99.7 \%$ \\
\hline
\end{tabular}

The conclusion drawn from the data gathered was that aqueous cleaning of LCCAs can replace the Freon vapor degreasing process with no detrimental consequences. 


\section{Discussion}

\section{Scope and Purpose}

The purpose of this project was to develop an environmentally friendly cleaning process for LCCA production to eliminate vapor degreasing with Freon. In developing a new, environmentally friendly cleaning process the following points were considered critical.

- Product reliability must remain at present level.

- Product yields must maintain current level.

- New cleaning must meet or exceed all environmental regulations.

\section{Activity}

\section{Background}

The passing of the Montreal Protocol requiring the elimination of CFCs led to the opportunity to closely evaluate the cleaning and handling procedures for semiconductor packaging. For the past ten years, cleaning had been done by vapor degreasing and ultrasonic rinsing in trichlorotrifluoroethane (CFC-113). This cleaning method was originally chosen because of the benign nature of the solvent coupled with the aggressive ultrasonic power to remove particles.

A cross-functional team of process, product, quality, material, and design lab engineers was assembled to investigate the cleaning issues for LCCAs. Using the TQ process, the team analyzed the current process, brainstormed possible alternatives, and then investigated their feasibility. To identify the root causes of contamination, investigation of stores packaging material and handling procedure for all components and assemblies was done. The major contaminarits of concern were lonic, particulate, and human debris.

To develop a new cleaning procedure, the group began by listing cleaning solutions based upon the environmental impact, operator safety, and cost of material. The cleaning solution ranking from most to least environmentally friendly was as follows:

1. No clean

2. Carbon dioxide snow (specifically for particulate)

3. DI water

4. DI water with surfactant

5. DI water with detergent

6. Organic solvent 
The process list was then established, based upon the mechanical agitation the process would impart on the product. Ranking from most mild to most aggressive was as follows:

1. Immersion

2. Immersion with stirring or dipping

3. Spray

4. Ultrasonic

5. Carbon dioxide snow

Combining the cleaning solution list with the mechanical agitation list gave 19 cleaning alternatives to potentially replace CFC vapor degreasing.

\section{Experimentation}

\section{Phase One}

The general cleanliness of the LCC packages in stores was investigated first to determine the best cleaning process for that component. Packages were stored in groups of $100 \mathrm{in}$ polypropylene containers. The containers were stored in nitrogen cabinets in the clean room. As jobs were released to the manufacturing floor, a container of 100 packages was removed from the nitrogen cabinet and sent with the die and lids. The packages were not typically handled individually by the storekeeper. If the storekeeper was required to handle any packages, a vacuum pencil or tweezers were used.

Two tests were run on the packages to measure their cleanliness as received on the manufacturing floor from stores. First was a flush and filter test to determine particulate contamination level. Second, Auger surface analysis was run to determine the surface condition and measure the cleaning ability of different cleaning processes. Concurrently with empty package testing, packages with die attached (having gone through eutectic die attach process) were being run through the same battery of tests.

Flush and filter testing was used to determine the quantity of particles 0.45 microns and larger which were present on a part. The procedure was to ultrasonically clean $(40 \mathrm{KHz})$ a part for 2 minutes. The part was contained in a test tube filled with $20 \mathrm{ml}$ of DI water filtered to 0.2 micron. The contents of the test tube are then vacuum filtered through 0.45 -micron filter paper. The paper is examined under $640 \mathrm{X}$ microscope to look for particles. All the work was done in a class 100 clean bench. Several blanks and base line runs were done before and during the testing to determine background particle contamination.

The general cleanliness of the combo lids in stores was investigated next. Combo lids were stored in groups of 100 in polyethylene bags inside polyethylene boxes and tightly packed with low-density polypropylene foam. The boxes of lids were stored in nitrogen cabinets in the clean room. As jobs were released to the manufacturing floor, a bag of 100 lids was removed from one of the polyethylene boxes and sent with the die and packages. The lids were not handled individually by the storekeeper. 
Three tests were run on the combo lids to measure the cleanliness of lids as received on the manufacturing floor from stores. First was a flush and filter test to determine particulate contarnination level. Next, Auger surface analysis was used to determine thickness of contaminating material and measure the cleaning ability of different cleaning processes. Third, GC-Mass spectrophotometry and gravineetric analysis were run using methylene chloride as the solvent to determine the type and quantity of contaminating organics.

Lastly, the equipment, handling, and the assembly process flow through lidding were investigated for contamination. Items such as die collets used on a die attach machine, waffle packs, conductive boxes, and handling fixtures were run through flush and filter testing. Assemblies were retested after die attach, wire bonding, pull testing, and storage, looking for particulate contamination.

\section{Phase Two}

From the results obtained in Phase 1 of the experimentation, the following cleaning processes were proposed for testing by the team.

1. Empty packages and packages containing die would be ultrasonically cleaned for two minutes in DI water, followed by a DI water spray rinse for 30 seconds, nitrogen blow off, and oven drying. To maintain the water integrity during the ultrasonic step, a cascading tank was used and the flow rate of the DI water was set at $0.5 \mathrm{gpm}$.

2. Packages with wire bonds attached would be flushed by flowing DI water in a cascading tank. The flow rate of the water was to be $2.0 \mathrm{gpm}$.

3. The contamination on the lids was considered minute, and lids were not to be cleaned.

With a new cleaning process established that met the environmentally friendly issue, test parts were run to verify that the process met with the established yield requirement. The yield requirements assessed were wire pull testing (NDPT), sealing, particle-induced noise detection (PIND) testing, and residual gas analysis (RGA). Also, to verify the reliability and to qualify the process for production, six lots of LCCAs were assembled and electrically and mechanically tested. Three lots were assembled using the current WR process with CFC-113 cleaning, and three lots were assembled by the WR process, substituting the new aqueous cleaning process for the CFC-113 process. The LCCAs contained a sample of all dice used for the common radar program. Testing included the following requirements.

1. The products were tested to the requirements of their respective product specifications.

2. Twenty pieces from each lot underwent high temperature reverse bias for 500 hours at $175^{\circ} \mathrm{C}$. No failures could occur when electrically tested.

3. Moisture analysis of five of each set of iwenty parts was run. Moisture level must be below 3000 ppm. 
4. The electrical data was analyzed for trends. Comparison of the data between aqueousclearisd and CFC-cleaned parts was done. Assuming no statistical difference between the two groups of data, aqueous cleanir.g was apprisved.

\section{Results}

\section{Phase One}

The overall results of the flush and filter testing can be seen in Table 2 .

Table 2. Number of Particles Captured During Flush and Filter Testing (Particle Size 0.45 Micron and Larger)

\begin{tabular}{|c|c|c|c|c|c|c|c|}
\hline $\begin{array}{c}\text { Test } \\
\text { Item }\end{array}$ & $\begin{array}{c}\text { Quaritity } \\
\text { Tested }\end{array}$ & $\begin{array}{c}\text { Gold } \\
\left(\# / \mathrm{cm}^{2}\right)\end{array}$ & $\begin{array}{c}\text { Ceramic } \\
\left(\# / \mathrm{cm}^{2}\right)\end{array}$ & $\begin{array}{c}\text { Silicon } \\
\left(\# / \mathrm{cm}^{2}\right)\end{array}$ & $\begin{array}{c}\text { Eutectic } \\
\left(\# / \mathrm{cm}^{2}\right)\end{array}$ & $\begin{array}{c}\text { Fibers } \\
\left(\# / \mathrm{cm}^{2}\right)\end{array}$ & $\begin{array}{c}\text { Other } \\
\left(\# / \mathrm{cm}^{2}\right)\end{array}$ \\
\hline $\begin{array}{c}\text { Empty } \\
\text { package }\end{array}$ & 10 & $100 \pm 25$ & $10 \pm 5$ & $\mathrm{ND}$ & $\mathrm{ND}$ & $2 \pm 2$ & $10 \pm 5$ \\
\hline $\begin{array}{c}\text { Die } \\
\text { attach } \\
\text { package }\end{array}$ & 10 & $50 \pm 15$ & $10 \pm 5$ & $15 \pm 10$ & $10 \pm 10$ & $\mathrm{ND}$ & $170 \pm 25$ \\
\hline $\begin{array}{c}\text { Wire } \\
\text { bond } \\
\text { package }\end{array}$ & 10 & $50 \pm 15$ & $\mathrm{ND}$ & $\mathrm{ND}$ & $\mathrm{ND}$ & $\mathrm{ND}$ & $1 \pm 1$ \\
\hline Li-'s & 12 & $\mathrm{ND}$ & $\mathrm{ND}$ & $\mathrm{ND}$ & $\mathrm{ND}$ & $\mathrm{ND}$ & $\mathrm{ND}$ \\
\hline Co!lets & 4 & $170 \pm 50$ & $80 \pm 30$ & $140 \pm 50$ & $50 \pm 25$ & $\mathrm{ND}$ & Infinite \\
\hline $\begin{array}{c}\text { Waffle } \\
\text { packs }\end{array}$ & 3 & $\mathrm{ND}$ & $\mathrm{ND}$ & $\mathrm{ND}$ & $\mathrm{ND}$ & $\mathrm{ND}$ & $400 \pm 100$ \\
\hline $\begin{array}{c}\text { Handling } \\
\text { fixtur:? }\end{array}$ & 3 & $\mathrm{ND}$ & $\mathrm{ND}$ & $\mathrm{ND}$ & $\mathrm{ND}$ & $\mathrm{ND}$ & $5 \pm 5$ \\
\hline
\end{tabular}

Tab!e 2 reprec ents average numbers of particles per square centimeter of filter paper recove; ed from each item, with an estimated range based on data collected from multiple runs. As can be seen from thie table, the most grossly contaminated parts were packages after die attach and the die attach sollets. The particles listed under the other category for die attach packages were a white iureign iriaterial. The possible sources of the material were residual adhesive on the die, waffle pack debris, or human debris.

4 larger number of gold flakes were shed from the packages during the ultrasonic cleaning. Subsequent ultrasonic cleaning (up to five runs) continued to yield very high gold flake counts, indicating that the package will continually shed gold plating during ultrasonic cleaning. Repeat cleaning of any package (empty, die attached, wire bonced) would result in only gold flakes being recovered. All other particles were removed during the first run. 
Surface analysis of packages received from stores was run to determine the organic contamination level. A sample of i6 LCCs was pulled from stores. Four were not cleaned, four were cleaned in alcohol, four were cleaned in DI water, and four were vapor degreased in CFC-113 (base line). The results shown in Figure 1 indicate that packages were free of organic contamination. Aill had carbon-to-gold ratios between 0.765 and 1.17 , indicating a clean surface. A carbon-to-gold peak ratio of zero $(0)$ would indicate an absolutely clean surface. The only way to achieve an absolutely clean surface is to plasma clean and never remove the part from the vacuum. Once exposed to factory air, carbon will deposit on the surface of the part. A carbon-to-gold ratio below 2.0 has less than $100 \AA$ of organic contamination.
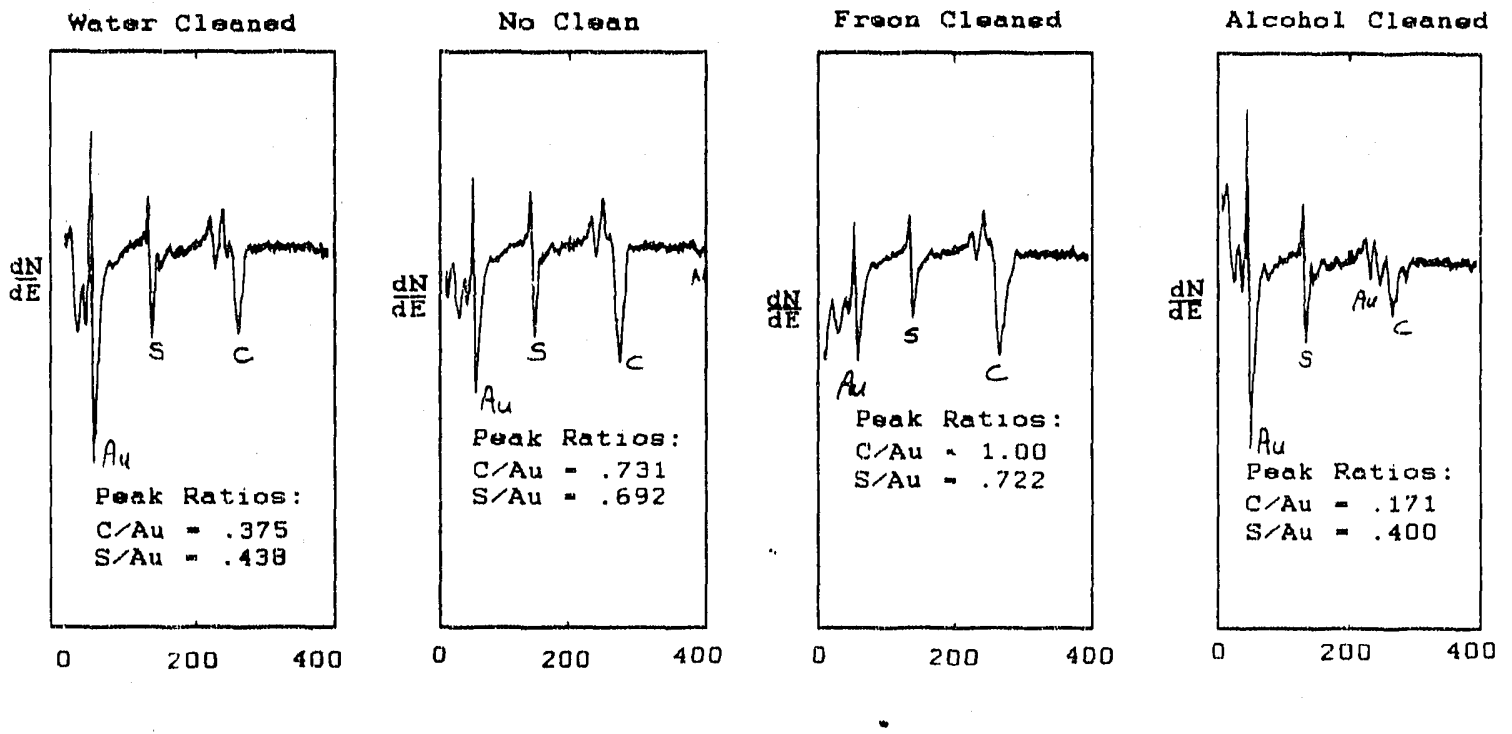

Figure 1. Auger Results for Ceramic Packages

Auger analysis was also run on a sample of nine lids, three uncleaned, three cleaned in alcohol, and three oxygen plasnia cleaned. The results of the Auger analysis can be seen in Figure 2. Uncleaned lids showed a relatively large carbon peak and smaller sulfur peak. The low energy gold peak is masked because of the carbon thickness. This is an indication of the organic contamination which was identified by GC-Mass spec. Lids which were alcohol cleaned showed great improvement over the uncleaned lids, and the oxygen plasma cleaned lids showed the best cleaning results. Carbon-to-gold peak ratios for the three samples of lids were 8.0 for uncleaned, 1.36 for alcohol cleaned, and 0.09 for oxygen plasma cleaned. 

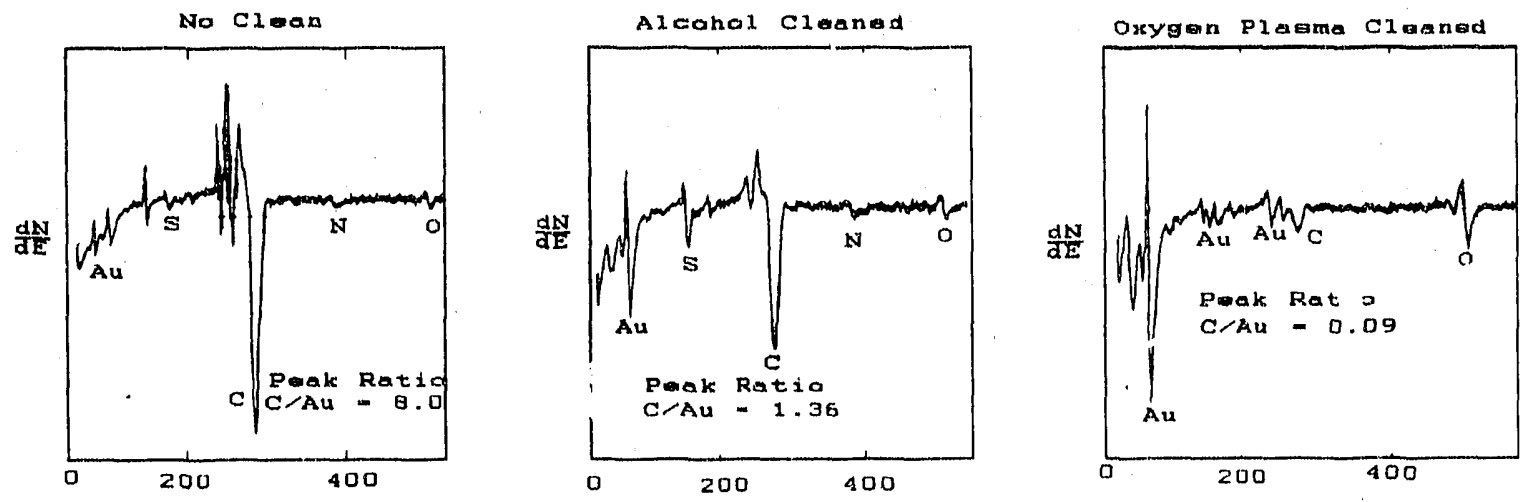

Figure 2. Auger Results for Kovar Lids

Two hundred lids were washed in methylene chloride. A very small sample of the solvent extract was analyzed and found to contain oleamide and erucamide (slip agents used $n n$ polyethylene bags). Also, trace amounts of low molecular weight polyethylene w'ere found.

The remainder of the solvent was evaporated, and gravimetric analysis of the residue was run. On the average, $0.01 \mathrm{mg}$ of residue is on each lid. This equates to a residue of $150 \mathrm{ppm}$ per lid by weight.

\section{Phase Two}

A sample of 150 LCCAs was assembled from excess material to test the yield potential for the new proposed cleaning process. The yield for critical processes is shown in Table 3.

Table 3. Yield Data for LCCAs Cleaned by Aqueous and Freon Processes

\begin{tabular}{|c|c|c|}
\hline & Aqueous & Freon (Historical) \\
\hline PIND (5 Runs) & $24 / 25(96 \%)$ & $96.4 \%$ \\
\hline NDPT & $150 / 150(100 \%)$ & $99.5 \%$ \\
\hline Sealing & $140 / 140(100 \%)$ & $99.7 \%$ \\
\hline
\end{tabular}

The residual gas analysis (RGA) results of five of the above parts is shown in Table 4.

Table 4. Internal Moisture Results for Aqueous- and Freon-Cleaned LCCAs Sealed the Same Day

\begin{tabular}{|c|c|c|}
\hline Sample & Aqueous & Freon \\
\hline 1 & 1,311 & 1,432 \\
\hline 2 & 1,494 & 1,268 \\
\hline 3 & 1,773 & 1,346 \\
\hline 4 & 1,114 & 888 \\
\hline 5 & 964 & 863 \\
\hline Avg. & 1,331 & 1,159 \\
\hline Std & 284 & 238 \\
\hline
\end{tabular}


Based on these results, the 600 LCCAs were assembled and tested as outlined above. Table 5 shows the results of the processing yields and Tables A1 through A 36 in the appendix show all the electrical test results and comparisons.

Table 5. Production Yields at Critical Points for Aqueous- and Freon-Cleaned LCCAs

\begin{tabular}{|c|c|c|}
\hline & Aqueous & Freon \\
\hline PIND (5 Runs) & $216 / 217(99.5 \%)$ & $239 / 240(99.6 \%)$ \\
\hline Sealing & $216 / 217(99.5 \%)$ & $239 / 242(98.8 \%)$ \\
\hline NDPT & $100 \%$ & $100 \%$ \\
\hline
\end{tabular}

The RGi results for these six Inte of LCCAs are shown in Table 6.

Table 6. Internal Moisture Results for Freon- and Aqueous-Cleaned LCCAs After 500-Hour HTRB

\begin{tabular}{|c|c|c|}
\hline PiN & Aqueous (ppm $\mathrm{H}_{2} \mathrm{O}$ ) & Freon $\left(\mathrm{ppm} \mathrm{H}_{2} \mathrm{O}\right)$ \\
\hline \multirow{5}{*}{$391289-01$} & 452 & 604 \\
\hline & $\overline{168}$ & $<100$ \\
\hline & 971 & 471 \\
\hline & 510 & 226 \\
\hline & 1,146 & 531 \\
\hline \multirow{5}{*}{$391291-01$} & 332 & 535 \\
\hline & 383 & 323 \\
\hline & 381 & 319 \\
\hline & 317 & 165 \\
\hline & 547 & 672 \\
\hline \multirow{5}{*}{$391376-01$} & 427 & 356 \\
\hline & 365 & 194 \\
\hline & 331 & 442 \\
\hline & 706 & 295 \\
\hline & $\overline{439}$ & 361 \\
\hline Avg. & 498 & 392 \\
\hline Std & 259 & 155 \\
\hline
\end{tabular}

\section{Accomplishments}

The overall results of the phase one testing indicate cleaning of empty packages and assemblies after die attach was required to remove particulate contamination. Organic contamination on packages was minute at most. Cleaning packages with an organic solvent gave no improvement to the surface condition. Therefore, the recommended cleaning process for packages was ultrasonic cleaning in DI water followed by a DI water spray rinse. However, ultrasonic cleaning of LCCAs containing wire bonds was NOT recommended. If cleaning was required after wire bonding, then assemblies should be flustied by flowing DI water in a 
cascading tank. DI water is the safest, most environmentally friendly solvent and has the added benefit of removing ionic contamination if it is present.

There was no sign of ionic or particulate contamination on the lids, only small amounts of organic residues. Oxygen plasma cleaning the lids or alcohol rinsing would remove the small amounts of contamination. However, the results of the assembly testing during phase two indicated that cleaning of lids was optional. Identical yield results were obtained for assemblies when the lids were cleaned or not. The sealing yield for cleaned and uncleaned lids, determined by fine and gross leak testing, had no statistically significant difference.

Based upon the mechanical assemblies built in phase two, aqueous cleaning of LCCAs was an acceptable process. No detrimental effects on product yields or reliability were found. The comparison of electrical data from the six lots of LCCAs in the appendix further proved that the aqueous cleaning process was a viable replacement for the CFC-113 vapor degreasing process.

Based on all the results of these experiments, it is recommended that the cleaning process for LCCAs with eutectically attached die be the following:

1. Empty packages and packages containing die would be ultrasonically cleaned for two minutes in DI water, followed by a DI water spray rinse for 30 seconds, nitrogen blow off and oven drying. To maintain the water integrity during the ultrasonic step, a cascading tank was used and the flow rate of the DI water was set at $0.5 \mathrm{gpm}$.

2. Packages with wire bonds attached would be flushed by flowing DI water in a cascading tank. The flow rate of the water was to be $2.0 \mathrm{gpm}$.

3. Tine contamination on the lids was considered minute, and lids were not to be cleaned. 
Appendix

\section{Electrical Test Data for Common Radar LCCAs}




\begin{tabular}{|c|c|c|c|c|c|c|}
\hline \multirow[t]{2}{*}{ TEST } & \multicolumn{3}{|c|}{ MINIMUM VALUE } & \multicolumn{3}{|c|}{ MAXIMUM VALUE } \\
\hline & Freon & DI Water & Delta & Freon & DI Water & Delta \\
\hline $\mathrm{ICBO}(\mathrm{nA}) @ \mathrm{VCB}=60 \mathrm{~V}$ & 0.4 & 0.51 & -0.11 & 3.8 & 7.7 & -3.9 \\
\hline $\mathrm{ICBO}(\mathrm{nA}) @ \mathrm{VCB}=75 \mathrm{~V}$ & 0.35 & 0.5 & -0.15 & 6.8 & 18 & -11.2 \\
\hline $\mathrm{IEBO}(\mathrm{nA}) @ \mathrm{VEB}=4 \mathrm{~V}$ & 0.05 & 0.01 & 0.05 & 9.3 & 2.1 & 7.2 \\
\hline $\operatorname{ICER}(\mathrm{nA}) @ \mathrm{VC}=50 \mathrm{~V}, \mathrm{R}=5 \mathrm{~K}$ & 0.29 & 0.34 & -0.05 & 2.5 & 6.9 & .4 .4 \\
\hline ICES (nA) @ VCE $=-50 \mathrm{~V}$ & 0.09 & 0.18 & -0.1 & 2.3 & 6.7 & -4.4 \\
\hline HFE @ VCE $=10 \mathrm{~V}, \mathrm{IC}=0.1 \mathrm{~mA}$ & 143.8 & 162.7 & -18.9 & 194.4 & 183.3 & 11.1 \\
\hline $\mathrm{HFE} @ \mathrm{VCE}=10 \mathrm{~V}, \mathrm{IC}=1 \mathrm{~mA}$ & 167.1 & 167.8 & -0.7 & 201 & 183.8 & 17.2 \\
\hline $\mathrm{HFE} @ \mathrm{VCE}=10 \mathrm{~V}, \mathrm{IC}=10 \mathrm{~mA}$ & 175.7 & 169.8 & 5.9 & 210 & 187.8 & 22.2 \\
\hline $\mathrm{HFE} @ \mathrm{VCE}=10 \mathrm{~V}, \mathrm{IC}=150 \mathrm{~mA}$ & 173.6 & 167.4 & 6.2 & 213 & 186.6 & 26.4 \\
\hline $\mathrm{HFE} @ \mathrm{VCE}=10 \mathrm{~V}, \mathrm{IC}=500 \mathrm{~mA}$ & 95.7 & 94.1 & 1.6 & 117.5 & 104.5 & 13 \\
\hline VCES(mV)@IC $=150 \mathrm{~mA}, \mathrm{IB}=15 \mathrm{~mA}$ & 147 & 151.5 & -4.5 & -161 & 164.5 & -3.5 \\
\hline VCES(mV)@IC $=500 \mathrm{~mA}, \mathbb{I B}=50 \mathrm{~mA}$ & 365.5 & 367 & 1.5 & 395 & 392.5 & 2.5 \\
\hline VBEON $(\mathrm{mV}) @ V C E=10 \mathrm{~V}, \mathrm{IC}=20 \mathrm{~mA}$ & 706 & 716 & -10 & 715.5 & 720 & -4.5 \\
\hline $\mathrm{VBEON}(\mathrm{mV}) @ \mathrm{VCE}=10 \mathrm{~V}, \mathrm{IC}=50 \mathrm{~mA}$ & 730.5 & 740.5 & .10 & 740.5 & 745 & -4.5 \\
\hline VBES $(\mathrm{mV}) @ \mathrm{IC}=150 \mathrm{~mA}, \mathrm{IB}=15 \mathrm{~mA}$ & 865.5 & 872 & -6.5 & 875 & 878.5 & -3.5 \\
\hline VBES(mV)@IC $=500 \mathrm{~mA}, \mathrm{IB}=50 \mathrm{~mA}$ & $1,082.5$ & $1,086.5$ & -4 & 1.105 .5 & 1,103 & 2.5 \\
\hline $\mathrm{ICBO}(\mathrm{nA}) @ \mathrm{VCB}=.50 \mathrm{~V}$ & 0.55 & 0.62 & -0.07 & 0.64 & 0.65 & -0.01 \\
\hline $1 C B O(n A) @ V C B=-60 V$ & 0.4 & 0.5 & -0.1 & 0.7 & 0.6 & 0.1 \\
\hline $\operatorname{ICER}(\mathrm{nA}) @ V C=-50 \mathrm{~V}, \mathrm{R}=5 \mathrm{~K}$ & 0.3 & 0.32 & -0.02 & 0.42 & 0.36 & 0.06 \\
\hline $\mathrm{IEBO}(\mathrm{nA}) @ \mathrm{VEB}=-4 \mathrm{~V}$ & 0.4 & 0.4 & 0 & 0.59 & 0.51 & 0.08 \\
\hline HFE @ VCE $=-10 \mathrm{~V}, \mathrm{IC}=-0.1 \mathrm{~mA}$ & 97.7 & 103.3 & -5.6 & 107 & 111.6 & -4.6 \\
\hline HFE @ VCE $=-10 \mathrm{~V}, \mathrm{IC}=-1 \mathrm{~mA}$ & 113.3 & 121.2 & -7.9 & 124.7 & 131.2 & -6.5 \\
\hline HFE @ VCE $=-10 \mathrm{~V}, \mathrm{IC}=-10 \mathrm{~mA}$ & 125 & 136.4 & -11.4 & 139.6 & 147.7 & -8.1 \\
\hline $\mathrm{HFE} @ \mathrm{VCE}=-10 \mathrm{~V}, \mathrm{IC}=-150 \mathrm{~mA}$ & 130.2 & 143.7 & -13.5 & 146.5 & 156.3 & -9.8 \\
\hline IFE@ VCE $=-10 \mathrm{~V}, \mathrm{IC}=-500 \mathrm{~mA}$ & 83.3 & 91.7 & -8.4 & 94.3 & 103.5 & -9.2 \\
\hline VCES(mV)@IC $=-150 \mathrm{~mA}, \mathrm{IB}=-15 \mathrm{~mA}$ & 161 & 156 & 5 & 169.5 & 165.5 & 4 \\
\hline VCES $(\mathrm{mV}) @ \mathrm{IC}=-500 \mathrm{~mA}, \mathrm{IB}=-50 \mathrm{~mA}$ & 407.5 & 391 & 16.5 & 441 & 42.1 & 20 \\
\hline VBEON(mV)@VCE $=-10 \mathrm{~V}, \mathrm{IC}=-20 \mathrm{~mA}$ & 711.5 & 715.5 & -4 & 718.5 & 720 & 1.5 \\
\hline VBEON $(\mathrm{mV}) @ V C E=-10 \mathrm{~V}, \mathrm{IC}=-50 \mathrm{~mA}$ & 735.5 & 738.5 & -3 & 742.5 & 743 & -0.5 \\
\hline VBES(mV)@IC $=-150 \mathrm{~mA}, \mathrm{IB}=-15 \mathrm{~mA}$ & 879.5 & 880.5 & -1 & 886 & 884.5 & 1.5 \\
\hline VBES(mV)@IC $=-500 \mathrm{nA}, \mathrm{IB}=-50 \mathrm{~mA}$ & $1,081.5$ & $1,072.5$ & 9 & $1,094.5$ & 1.083 .5 & 11 \\
\hline $\mathrm{IR}(\mu \mathrm{A}) @ \mathrm{VR}=6 \mathrm{~V}$ & 1.09 & 0.87 & 0.22 & 2.24 & 1.79 & $0 . \overline{45}$ \\
\hline $\mathrm{IR}(\mu \mathrm{A}) @ \mathrm{VR}=36 \mathrm{~V}$ & 4.47 & 3.67 & 0.8 & 13.8 & 16 & -2.2 \\
\hline $\mathrm{VF}(\mathrm{mV}) @ \mathrm{IF}=2 \mathrm{~mA}$ & 196.5 & 212.5 & .16 & 214 & 218 & -4 \\
\hline $\mathrm{VF}(\mathrm{mV}) @ \mathrm{IF}=10 \mathrm{~mA}$ & 240 & 255.5 & 15.5 & 256.5 & 261.5 & -5 \\
\hline $\mathrm{VF}(\mathrm{mV}) @ \mathrm{IF}=10 \mathrm{gm \textrm {m }}$ & 313 & 327.5 & -14.5 & 330.5 & 333 & -2.5 \\
\hline $\mathrm{VF}(\mathrm{mV}) @ \mathrm{IF}=19$ & 486.5 & 493 & -6.5 & 517.5 & 524.5 & .7 \\
\hline $\mathrm{VF}(\mathrm{mV}) @ \mathrm{IF}=2 \mathrm{i}$ & 629.5 & 62.9 .5 & 0 & 678 & 692 & -14 \\
\hline $\mathrm{IR}(\mu \mathrm{A}) @ \mathrm{VR}=6 \mathrm{~V}$ & 1.18 & 0.85 & 0.33 & 2.75 & 1.54 & 1.21 \\
\hline $\mathrm{IR}(\mu \mathrm{A}) @ \mathrm{VR}=36 \mathrm{~V}$ & 4.56 & -3.6 & 0.96 & 15.3 & 20.4 & -5.1 \\
\hline $\mathrm{VF}(\mathrm{mV}) @ \mathrm{IF}=2 \mathrm{~mA}$ & 192 & 204 & -12 & 211.5 & 219 & -7.5 \\
\hline $\mathrm{VF}(\mathrm{mA}) @ \mathrm{IF}=10 \mathrm{~mA}$ & 235.5 & 247 & -11.5 & 255.5 & 262 & -6.5 \\
\hline $\mathrm{VF}(\mathrm{mV}) @ \mathrm{IF}=100 \mathrm{~mA}$ & 307.5 & 318.5 & -11 & 327.5 & 335.5 & .6 \\
\hline $\mathrm{VF}(\mathrm{mV}) @ \mathrm{IF}=1 \mathrm{~A}$ & 479.5 & 486.5 & -7 & 524.5 & 505.5 & 19 \\
\hline $\mathrm{VF}(\mathrm{mV}) @ \mathrm{IF}=2 \mathrm{~A}$ & 621.5 & 625.5 & -4 & 688 & 50.5 & 37.5 \\
\hline
\end{tabular}


TABLE A2: AVERAGE AND STANDARD DEVIATION OF INITAL ELECTRICAL TEST DATA FOR M-53

\begin{tabular}{|c|c|c|c|c|c|c|}
\hline \multirow[t]{2}{*}{ TEST } & \multicolumn{3}{|c|}{ AVERAGE } & \multicolumn{3}{|c|}{ STANDARD DEVIATION } \\
\hline & Freon & DI Water & Delta & Freon & DI Water & Delta \\
\hline $\mathrm{ICBO}(\mathrm{nA}) @ \mathrm{VCB}=60 \mathrm{~V}$ & 0.51 & 0.77 & -0.26 & 0.4 & 1 & -0.6 \\
\hline $\mathrm{ICBO}(\mathrm{nA}) @ \mathrm{VCB}=75 \mathrm{~V}$ & 0.59 & 1.2 & -0.61 & 0.74 & 2.7 & -1.96 \\
\hline IEBO $(n A) @ V E B=4 V$ & 0.23 & 0.06 & 0.17 & 1.1 & 0.29 & 0.81 \\
\hline $\operatorname{ICER}(n A) @ V C=50 V, R=5 K$ & 0.38 & 0.52 & -0.14 & 0.25 & 0.78 & -0.53 \\
\hline $\operatorname{ICES}(\mathrm{nA}) @ \mathrm{VCE}=-50 \mathrm{~V}$ & 0.22 & 0.37 & 0.15 & 0.25 & 0.78 & -0.53 \\
\hline $\mathrm{HFE} @ \mathrm{VCE}=10 \mathrm{~V}, \mathrm{IC}=0.1 \mathrm{~mA}$ & 179.64 & 176.2 & 3.44 & 10.96 & 4.16 & 6.8 \\
\hline $\mathrm{HFE} @ \mathrm{VCE}=10 \mathrm{~V}, \mathrm{IC}=1 \mathrm{~mA}$ & 188.75 & 175.42 & 13.33 & 8.95 & 4.35 & 4.6 \\
\hline $\mathrm{HFE} @ \mathrm{VCE}=10 \mathrm{~V}, \mathrm{IC}=10 \mathrm{~mA}$ & 198.54 & 177.88 & 20.66 & 10.77 & 4.83 & 5.94 \\
\hline HFE @ VCE $=10 \mathrm{~V}, I C=150 \mathrm{~mA}$ & 198.75 & 175.88 & 22.87 & 11.5 & 5.25 & 6.24 \\
\hline $\mathrm{HFE} @ \mathrm{VCE}=10 \mathrm{~V}, \mathrm{IC}=500 \mathrm{~mA}$ & $106 . \overline{44}$ & 98.3 & 8.14 & 5.91 & 2.7 & 3.21 \\
\hline $\mathrm{VCES}(\mathrm{mV}) @ \mathrm{IC}=150 \mathrm{~mA}, \mathrm{IB}=15 \mathrm{~mA}$ & 153.77 & 159.45 & -5.68 & 3.53 & 3.54 & -0.01 \\
\hline VCES $(\mathrm{mV}) @ \mathrm{IC}=-500 \mathrm{~mA}, \mathrm{IB}=-50 \mathrm{~mA}$ & 382.07 & 383.23 & -1.16 & 6.05 & 6.32 & -0.27 \\
\hline VBEON(mV)@VCE $=10 \mathrm{~V}, \mathrm{IC}=20 \mathrm{~mA}$ & 709.24 & 718.1 & .8 .86 & 2.44 & 0.86 & 1.58 \\
\hline VBEON $(\mathrm{mV}) @ \mathrm{VCE}=10 \mathrm{~V}, \mathrm{IC}=50 \mathrm{~mA}$ & 734.13 & 742.75 & -8.62 & 2.52 & 0.98 & 1.54 \\
\hline VBES $(\mathrm{mV}) @ \mathrm{IC}=150 \mathrm{~mA}, \mathrm{IB}=15 \mathrm{~mA}$ & 870.61 & 875.28 & -4.67 & 1.98 & 1.24 & 0.74 \\
\hline $\mathrm{VBES}(\mathrm{mV}) @ \mathrm{IC}=500 \mathrm{~mA}, \mathrm{IB}=50 \mathrm{~mA}$ & $1,095.1$ & $1,096.1$ & -1 & 4.8 & 3.16 & 1.64 \\
\hline$I C B O(n A) @ V C B=-50 V$ & 0.61 & 0.63 & -0.02 & 0.02 & 0.01 & 0.01 \\
\hline $\mathrm{ICBO}(\mathrm{nA}) @ \mathrm{VCB}=.60 \mathrm{~V}$ & 0.56 & 0.57 & -0.01 & 0.06 & 0.04 & 0.02 \\
\hline $\operatorname{ICER}(\mathrm{nA}) @ V C=-50 \mathrm{~V}, \mathrm{R}=5 \mathrm{~K}$ & 0.37 & 0.33 & 0.04 & 0.03 & 0.01 & 0.01 \\
\hline IEBO (nA)@VEB=-4V & 0.53 & 0.46 & 0.07 & 0.04 & 0.02 & 0.01 \\
\hline HFE @ VCE $=-10 \mathrm{~V}, \mathrm{IC}=-0.1 \mathrm{~mA}$ & 104.26 & 108.69 & -4.43 & 1.76 & 1.74 & 0.02 \\
\hline $\mathrm{HFE} @ \mathrm{VCE}=-10 \mathrm{~V}, \mathrm{IC}=-1 \mathrm{~mA}$ & 120.77 & 127.27 & -6.5 & 2.21 & .05 & 0.16 \\
\hline HFE @ VCE $=-10 \mathrm{~V}, I \mathrm{C}=-10 \mathrm{~mA}$ & 134.59 & 143.09 & -8.5 & 2.73 & 2.4 & 0.32 \\
\hline HFE @ VCE $=-10 \mathrm{~V}, I \mathrm{C}=-150 \mathrm{~mA}$ & 140.84 & 150.44 & -9.6 & 3.1 & 3.5 & -0.4 \\
\hline HFE @ VCE $=-10 \mathrm{~V}, \mathrm{IC}=-500 \mathrm{~mA}$ & 91.82 & 99.81 & -7.99 & 2.76 & 2.9 & -0.14 \\
\hline VCES $(\mathrm{mV}) @ \mathrm{IC}=-150 \mathrm{~mA}, \mathrm{IB}=-15 \mathrm{~mA}$ & 164.3 & 159.49 & 4.81 & 1.87 & 2.1 & -0.23 \\
\hline $\mathrm{VCES}(\mathrm{mV}) @ \mathrm{IC}=-500 \mathrm{~mA}, \mathrm{IB}=-50 \mathrm{~mA}$ & 419.24 & 402.25 & 16.99 & 7.59 & 6.45 & 1.14 \\
\hline VBEON(mV)@VCE $=-10 \mathrm{~V}, I \mathrm{IC}=-20 \mathrm{~mA}$ & 714.8 & 717.61 & 2.81 & 1.29 & 0.88 & 0.42 \\
\hline VBEON $(\mathrm{mV}) @ \mathrm{VCE}=-10 \mathrm{~V}, \mathrm{IC}=-50 \mathrm{~mA}$ & 738.09 & 740.32 & -2.23 & 1.31 & 0.85 & 0.46 \\
\hline $\operatorname{VBES}(\mathrm{m} V) @ \mathrm{IC}=-150 \mathrm{~mA}, \mathrm{IB}=-15 \mathrm{~mA}$ & 882.38 & 882.4 & -0.02 & 1.29 & 0.86 & 0.43 \\
\hline VBES(mV)@IC $=-500 \mathrm{~mA}, \mathrm{IB}=-50 \mathrm{~mA}$ & $1,087.6$ & $1,077.6$ & 10 & 3.28 & 2.37 & 0.91 \\
\hline IR $(\mu \mathrm{A}) @ \mathrm{VR}=6 \mathrm{~V}$ & 1.6 & 1 & 0.6 & 0.21 & 0.12 & 0.09 \\
\hline $\mathrm{IR}(\mu \mathrm{A}) @ \mathrm{VR}=36 \mathrm{~V}$ & 6.36 & 4.29 & 2.07 & 1.48 & 1.63 & -0.15 \\
\hline $\mathrm{VF}(\mathrm{mV}) @ \mathrm{IF}=2 \mathrm{~mA}$ & 205.26 & 215.41 & -10.15 & 2.99 & 1.13 & 1.86 \\
\hline$V F(m A) @ I F=10 \mathrm{~mA}$ & 248.63 & 258.27 & -9.64 & 2.96 & 1.35 & 1.61 \\
\hline $\mathrm{VF}(\mathrm{mV}) @ \mathrm{IF}=100 \mathrm{~mA}$ & 321.42 & 329.8 & -8.38 & 2.94 & 1.3 & 1.64 \\
\hline $\mathrm{VF}(\mathrm{mV}) @ \mathrm{IF}=1 \mathrm{~A}$ & 497.33 & 500.11 & -2.78 & 4.82 & 3.76 & 1.06 \\
\hline $\mathrm{VF}(\mathrm{mV}) @ \mathrm{IF}=2 \mathrm{~A}$ & 645.68 & 642.08 & 3.6 & 8.1 & 7.23 & 0.87 \\
\hline $\mathrm{IR}(\mu \mathrm{A}) @ \mathrm{VR}=6 \mathrm{~V}$ & 1.62 & 1.01 & 0.61 & 0.31 & 0.12 & 0.18 \\
\hline $\mathrm{IR}(\mu \mathrm{A}) @ \mathrm{VR}=36 \mathrm{~V}$ & 6.42 & 4.52 & 1.9 & 1.98 & 1.95 & 0.03 \\
\hline$V F(m V) @ I F=2 m A$ & 204.84 & 214.79 & -9.95 & 3.93 & 2.83 & 1.1 \\
\hline$V F(m V) @ \mathrm{IF}=10 \mathrm{~mA}$ & 248.25 & 257.75 & -9.5 & 3.88 & 2.83 & 1.05 \\
\hline$V F(m V) @ I F=100 \mathrm{~mA}$ & 320.7 & 329.27 & -8.57 & 3.8 & 2.87 & 0.93 \\
\hline $\mathrm{VF}(\mathrm{mV}) @ \mathrm{IF}=1 \mathrm{~A}$ & 494.27 & 499.01 & -4.74 & 5.94 & 4.19 & 1.75 \\
\hline$V F(m V) @ I F=2 A$ & 639.25 & 640.16 & -0.91 & 9.05 & 6.14 & 2.91 \\
\hline
\end{tabular}




\begin{tabular}{|c|c|c|c|c|c|c|}
\hline \multirow[t]{2}{*}{ TEST } & \multicolumn{3}{|c|}{ MINIMUM VALUE } & \multicolumn{3}{|c|}{ MAXIMUM VALUE } \\
\hline & Freon & DI Water & Delta & Freon & DI Water & De!:a \\
\hline $\mathrm{ICBO}(\mathrm{A}) @ \mathrm{VCB}=60 \mathrm{~V}$ & 0.36 & 3 & -2.64 & 3 & 7.7 & -4.7 \\
\hline$\overline{I C B O}(\mathrm{nA}) @ V C B=75 \mathrm{~V}$ & 0.3 & 0.5 & -0.2 & 5.5 & 18 & -12.5 \\
\hline IEBO(nA)@VEB=4V & 0.01 & 0.01 & 0 & 8.7 & 2.1 & 6.6 \\
\hline $\operatorname{ICER}(n \mathrm{~A}) @ V C=50 \mathrm{~V}, R=5 \mathrm{~K}$ & 0.18 & 0.34 & 0.16 & 1.9 & 6.9 & -5 \\
\hline $\operatorname{ICES}(\mathrm{nA}) @ \mathrm{VCE}=-50 \mathrm{~V}$ & 0.11 & 0.18 & -0.07 & 1.7 & 6.7 & -5 \\
\hline $\mathrm{HFE} @ \mathrm{VCE}=10 \mathrm{~V}, \mathrm{IC}=0.1 \mathrm{~mA}$ & 140.8 & 162.7 & -21.9 & 192.5 & 183.3 & 9.2 \\
\hline HFE @ VCE $=10 \mathrm{~V}, \mathrm{IC}=1 \mathrm{~mA}$ & 163.5 & 167.8 & -4.3 & 199.2 & 183.8 & 15,4 \\
\hline $\mathrm{HFE} @ \mathrm{VCE}=10 \mathrm{~V}, \mathrm{IC}=10 \mathrm{~mA}$ & 173.6 & 169.8 & 3.8 & 209 & 187.8 & 21.2 \\
\hline HFE @ VCE $=10 \mathrm{~V}, \mathrm{IC}=150 \mathrm{~mA}$ & 173.6 & 167.4 & 6.2 & 213 & 186.6 & 26.4 \\
\hline HFE @ VCE $=10 \mathrm{~V}, \mathrm{IC}=500 \mathrm{~mA}$ & 95.6 & 94.1 & 1.5 & 117 & 104.5 & 12.5 \\
\hline VCES(mV)@IC $=150 \mathrm{~mA}, I B=15 \mathrm{~mA}$ & 146.5 & 151.5 & -5 & 160.5 & 164.5 & -4 \\
\hline VCES(mV)@IC $=500 \mathrm{~mA}, \mathrm{IB}=50 \mathrm{~mA}$ & 363.5 & 367 & -3.5 & 394 & 392.5 & 1.5 \\
\hline VBEON $(\mathrm{mV}) @ V C E=10 \mathrm{~V}, I \mathrm{C}=20 \mathrm{~mA}$ & 705 & 716 & -11 & 719 & 720 & -1 \\
\hline VBEON $(\mathrm{mV}) @ V C E=10 \mathrm{~V}, \mathrm{IC}=50 \mathrm{~mA}$ & 729 & 740.5 & -11.5 & 745 & 745 & 0 \\
\hline VBES(mV)@IC $=150 \mathrm{~mA}, 1 \mathrm{~B}=15 \mathrm{~mA}$ & 864.5 & 872 & -7.5 & 877.5 & 878.5 & -1 \\
\hline $\mathrm{VBES}(\mathrm{mV}) @ \mathrm{IC}=500 \mathrm{~mA}, \mathrm{IB}=50 \mathrm{~mA}$ & $1,081.5$ & $1,086.5$ & -5 & 1104.5 & 1,103 & 1.5 \\
\hline$I C B O(n A) @ V C B=-50 V$ & 0.56 & 0.62 & -0.06 & 0.64 & 0.65 & -0.01 \\
\hline $\mathrm{ICBO}(\mathrm{nA}) @ \mathrm{VCB}=-60 \mathrm{~V}$ & 0,4 & 0.5 & -0.1 & 0.65 & 0.6 & 0.05 \\
\hline $\operatorname{ICER}(\mathrm{nA}) @ \mathrm{VC}=-50 \mathrm{~V}, \mathrm{R}=5 \mathrm{~K}$ & 0.32 & 0.32 & 0 & 0.4 & 0.36 & 0.04 \\
\hline $\mathrm{IEBO}(\mathrm{nA}) @ \mathrm{VEB}=-4 \mathrm{~V}$ & 0.42 & 0.4 & 0.02 & 0.57 & 0.51 & 0.06 \\
\hline HFE @ VCE $=-10 \mathrm{~V}, I \mathrm{C}=-0.1 \mathrm{~mA}$ & 96.4 & 103.3 & -6.9 & 106.2 & 111.6 & -5.4 \\
\hline $\mathrm{HFE} @ \mathrm{VCE}=-10 \mathrm{~V}, \mathrm{IC}=-1 \mathrm{~mA}$ & 111.4 & 121.2 & -9.8 & 123.3 & 131.2 & -7.9 \\
\hline $\mathrm{HFE} @ \mathrm{VCE}=-10 \mathrm{~V}, \mathrm{IC}=-10 \mathrm{~mA}$ & 123.6 & 136.4 & -12.8 & 137.9 & 147.7 & -9.8 \\
\hline $\mathrm{HFE} @ \mathrm{VCE}=-10 \mathrm{~V}, \mathrm{IC}=-150 \mathrm{~mA}$ & 130.2 & 143.7 & -13.5 & 144.2 & 156.3 & -12.1 \\
\hline HFE @ VCE $=-10 \mathrm{~V}, \mathrm{IC}=-500 \mathrm{~mA}$ & 82.2 & 91.7 & -9.5 & 93.9 & 103.5 & -9.6 \\
\hline $\mathrm{VCES}(\mathrm{mV}) @ \mathrm{IC}=-150 \mathrm{~mA}, \mathrm{IB}=-15 \mathrm{~mA}$ & 162 & 156 & 6 & 170.5 & 165.5 & 5 \\
\hline VCES(mV)@IC $=-500 \mathrm{~mA}, \mathrm{IB}=-50 \mathrm{~mA}$ & 408.5 & 391 & 17.5 & 445 & 421 & 24 \\
\hline VBEON $(\mathrm{mV}) @ V C E=-10 \mathrm{~V}, \mathrm{IC}=-20 \mathrm{~mA}$ & 711.5 & 715.5 & -4 & 721 & 720 & 1 \\
\hline VBEON $(\mathrm{mV}) @ V C E=-10 \mathrm{~V}, \mathrm{IC}=-50 \mathrm{~mA}$ & 735 & 738.5 & -3.5 & 744.5 & 743 & 1.5 \\
\hline VBES $(\mathrm{mV}) @ \mathrm{IC}=-150 \mathrm{~mA}, \mathrm{IB}=-15 \mathrm{~mA}$ & 879.5 & 880.5 & -1 & 887.5 & 884.5 & 3 \\
\hline VBES $(\mathrm{mV}) @ \mathrm{IC}=-500 \mathrm{~mA}, \mathrm{IB}=-50 \mathrm{~mA}$ & 1,082 & $1,072.5$ & 9.5 & 1096 & $1,083.5$ & 12.5 \\
\hline $\mathrm{IR}(\mu \mathrm{A}) @ \mathrm{VR}=6 \mathrm{~V}$ & 0.92 & 2.07 & -1.15 & 2.07 & 1.79 & 0.28 \\
\hline IR $(\mu \mathrm{A}) @ \mathrm{VR}=36 \mathrm{~V}$ & 3.94 & 3.67 & $0 . \overline{27}$ & 15 & 16 & -1 \\
\hline $\mathrm{VF}(\mathrm{mV})(\mathrm{IF}=2 \mathrm{~mA}$ & 198 & 212.5 & -14.5 & 217.5 & 218 & -0.5 \\
\hline $\mathrm{VF}(\mathrm{miV}) @ \mathrm{IF}=10 \mathrm{~mA}$ & 241.5 & 255.5 & -14 & 260 & 261.5 & -1.5 \\
\hline $\mathrm{VF}(\mathrm{mV}) @ \mathrm{IF}=100 \mathrm{~mA}$ & 314.5 & 327.5 & -13 & 332.5 & 333 & -0.5 \\
\hline$V F(m V) @ I F=1 A$ & 492.5 & 493 & -0.5 & 507.5 & 524.5 & -17 \\
\hline$V F(m V) @ I F=2 A$ & 639.5 & 629.5 & 10 & 659.5 & 692 & -32.5 \\
\hline $\mathrm{IR}(\mu \mathrm{A}) @ \mathrm{VR}=6 \mathrm{~V}$ & 1.04 & 0.85 & 0.19 & 2.48 & 1.54 & 0.94 \\
\hline IR $(\mu \mathrm{A}) @ \mathrm{VR}=36 \mathrm{~V}$ & 4.23 & 3.6 & 0.63 & 13.5 & 20.4 & -6.9 \\
\hline $\mathrm{VF}(\mathrm{mV}) @ \mathrm{IF}=2 \mathrm{~mA}$ & 194 & 204 & -10 & 215 & 219 & -4 \\
\hline $\mathrm{VF}(\mathrm{mV}) @ \mathrm{IF}=10 \mathrm{~mA}$ & 237.5 & 247 & -9.5 & 257.5 & 262 & -4.5 \\
\hline $\mathrm{VF}(\mathrm{mV}) @ \mathrm{IF}=100 \mathrm{~mA}$ & 309.5 & 318.5 & -9 & 329.5 & 333.5 & -4 \\
\hline $\mathrm{VF}(\mathrm{mV}) @ \mathrm{IF}=1 \mathrm{~A}$ & 479.5 & 486.5 & -7 & 520 & 505.5 & 14.5 \\
\hline $\mathrm{VF}(\mathrm{mV}) @ \mathrm{IF}=2 \mathrm{~A}$ & 617 & 625.5 & -8.5 & 690.5 & 650.5 & 40 \\
\hline
\end{tabular}




\begin{tabular}{|c|c|c|c|c|c|c|}
\hline \multicolumn{7}{|c|}{ TABLE A4: AVERAGE AND STANDARD DEVIATION OF POST HTRB ELECTR AL TEST DATA FOR M-53 } \\
\hline \multirow[t]{2}{*}{ TEST } & \multicolumn{3}{|c|}{ AVERAGE VALUE } & \multicolumn{3}{|c|}{ STANDARD DEVIATION } \\
\hline & Freon & DI Water & Delta & Freon & DI Water & Delta \\
\hline $\mathrm{ICBO}(\mathrm{nA}) @ \mathrm{VCB}=60 \mathrm{~V}$ & 0.49 & 0.77 & -0.28 & 0.32 & 1 & -0.68 \\
\hline $\mathrm{ICBO}(\mathrm{nA}) @ \mathrm{VCB}=75 \mathrm{~V}$ & 0.58 & 1.2 & -0.62 & 0.62 & 2.7 & -2.08 \\
\hline $\operatorname{IEBO}(\mathrm{nA}) @ \mathrm{VEB}=4 \mathrm{~V}$ & 0.21 & 0.06 & 0.15 & 1.1 & 0.29 & 0.81 \\
\hline ICER (nA)@ VC $=50 \mathrm{~V}, \mathrm{R}=5 \mathrm{~K}$ & 0.35 & 0.52 & -0.17 & 0.2 & 0.78 & -0.58 \\
\hline ICES (nA)@ VCE $=-50 V$ & 0.2 & 0.37 & $-0,17$ & 0.21 & 0.78 & -0.57 \\
\hline $\mathrm{HFE} @ \mathrm{VCE}=10 \mathrm{~V}, \mathrm{IC}=0,1 \mathrm{~mA}$ & 177.89 & 176.2 & 1.69 & 11,18 & 4.16 & 7.02 \\
\hline $\mathrm{HFE} @ \mathrm{VCE}=10 \mathrm{~V}, \mathrm{IC}=1 \mathrm{~mA}$ & 186.77 & 175.42 & 11.35 & 9.15 & 4.35 & 4,8 \\
\hline $\mathrm{HFE} @ \mathrm{VCE}=10 \mathrm{~V}, \mathrm{IC}=10 \mathrm{~mA}$ & 196.67 & 177.88 & 18.79 & 10.72 & 4.83 & 5.88 \\
\hline $\mathrm{HFE} @ \mathrm{VCE}=10 \mathrm{~V}, \mathrm{IC}=150 \mathrm{~mA}$ & 197.28 & 175.88 & 21.4 & 11.73 & 5.25 & 6.48 \\
\hline $\mathrm{HFE} @ \mathrm{VCE}=10 \mathrm{~V}, \mathrm{IC}=500 \mathrm{~mA}$ & 106.42 & 98.3 & 8.12 & 5.96 & 2.7 & 3.26 \\
\hline VCES(mV)@IC $=150 \mathrm{~mA}, I B=15 \mathrm{~mA}$ & 153.08 & 159.45 & -6.37 & 3.52 & 3.54 & -0.02 \\
\hline $\mathrm{VCES}(\mathrm{mV}) @ \mathrm{IC}=500 \mathrm{~mA}, \mathrm{IB}=50 \mathrm{~mA}$ & 380.4 & 383,23 & -2.83 & 6.03 & 6.32 & .0 .29 \\
\hline VBEON $(\mathrm{mV}) @ V C E=10 \mathrm{~V}, \mathrm{IC}=20 \mathrm{~mA}$ & 711.23 & 718.1 & -6.87 & 3.28 & 0.86 & 2.42 \\
\hline VBEON $(\mathrm{mV}) @ V C E=10 \mathrm{~V}, \mathrm{IC}=50 \mathrm{~mA}$ & 736.09 & 742.75 & .6 .66 & 3.33 & 0.98 & 2.35 \\
\hline VBES(mV)@IC $=150 \mathrm{~mA}, I B=15 \mathrm{~mA}$ & 871.67 & 875.28 & -3.61 & 2.56 & 1.24 & 1.32 \\
\hline $\mathrm{VBES}(\mathrm{mV}) @ \mathrm{IC}=500 \mathrm{~mA}, 1 \mathrm{~B}=50 \mathrm{~mA}$ & 1,095 & $1,096.1$ & -1.1 & 4.89 & 3.16 & 1.73 \\
\hline$I C B O(n A) @ V C B=-50 V$ & 0.58 & 0.63 & -0.05 & 0.02 & 0.01 & 0.01 \\
\hline $\mathrm{ICBO}(\mathrm{nA}) @ \mathrm{VCB}=.60 \mathrm{~V}$ & 0.53 & 0.57 & -0.04 & 0.07 & 0.04 & 0.03 \\
\hline ICER (nA)@VC $=-50 V, R=5 \mathrm{~K}$ & 0.35 & 0.33 & 0.02 & 0.03 & 0.01 & 0.02 \\
\hline $\mathrm{IEBO}(\mathrm{nA}) \otimes \mathrm{VEB}=-4 \mathrm{~V}$ & 0.51 & 0.46 & 0.05 & 0.03 & 0.02 & 0.01 \\
\hline HFE @ VCE $=-10 \mathrm{~V}, I \mathrm{C}=-0.1 \mathrm{~mA}$ & 102.85 & 108.69 & -5.84 & 1.9 & 1.73 & 0.17 \\
\hline $\mathrm{HFE} @ \mathrm{VCE}=-10 \mathrm{~V}, \mathrm{IC}=-1 \mathrm{~mA}$ & 119.26 & 127.27 & -8.01 & 2.3 & 2.05 & 0.25 \\
\hline $\mathrm{HFE} @ \mathrm{VCE}=-10 \mathrm{~V}, \mathrm{LC}=-13 \mathrm{~mA}$ & 132.88 & 143.09 & -10.21 & 2.8 & 2.4 & 0.4 \\
\hline $\mathrm{HFE} @ \mathrm{VCE}=-10 \mathrm{~V}, \mathrm{IC}=-150 \mathrm{~mA}$ & 138.85 & 150.44 & -11.59 & 3.07 & 3.5 & -0.43 \\
\hline $\mathrm{HFE} @ \mathrm{VCE}=-10 \mathrm{~V}, \mathrm{IC}=-500 \mathrm{~mA}$ & 90.82 & 99.81 & -8.99 & 2.83 & 2.9 & .0 .07 \\
\hline VCES(mV)@IC $=-150 \mathrm{~mA}, \mathrm{IB}=-15 \mathrm{~mA}$ & 164.86 & 159.49 & 5.37 & 1.88 & 2.1 & -0.22 \\
\hline $\operatorname{VCES}(\mathrm{mV}) @ \mathrm{IC}=-500 \mathrm{~mA}, \mathrm{IB}=-50 \mathrm{~mA}$ & 421.03 & 402.25 & 18.78 & 7,99 & 6.45 & 1.54 \\
\hline VBEON $(\mathrm{mV}) @ V C E=-10 \mathrm{~V}, \mathrm{IC}=-20 \mathrm{~mA}$ & 716.58 & 717.61 & -1.03 & 2.37 & 0.88 & 1.51 \\
\hline VBEON $(\mathrm{mV}) @ V C E=-10 \mathrm{~V}, 1 \mathrm{C}=-50 \mathrm{~mA}$ & 740.11 & 740.32 & -0.21 & 2.33 & 0.85 & 1.48 \\
\hline VBES $(\mathrm{InV}) @ \mathrm{IC}=-150 \mathrm{~mA}, \mathrm{IB}=-15 \mathrm{~mA}$ & 883.65 & 882.4 & 1.25 & 1.9 & 0.86 & 1.04 \\
\hline VBES $(\mathrm{mV}) @ I C=-500 \mathrm{~mA}, I B=-50 \mathrm{~mA}$ & $1,088.8$ & $1,077.6$ & 11.2 & 3.49 & 2.37 & 1.12 \\
\hline $\mathrm{IR}(\mu \mathrm{A}) @ \mathrm{VR}=6 \mathrm{~V}$ & 1.47 & 1 & 0.47 & 0.23 & 0.12 & 0.11 \\
\hline $\mathrm{IR}(\mu \mathrm{A}) @ \mathrm{VR}=36 \mathrm{~V}$ & 5.97 & 4.29 & 1.68 & 1.46 & 1.63 & -0.17 \\
\hline $\mathrm{VF}(\mathrm{mV}) @ \mathrm{IF}=2 \mathrm{~mA}$ & 207.13 & 215.41 & -8.28 & 3.46 & 1.13 & 2.33 \\
\hline $\mathrm{VF}(\mathrm{mV}) @ \mathrm{IF}=10 \mathrm{~mA}$ & 250,49 & 258.27 & -7.78 & 3.29 & 1.35 & 1.94 \\
\hline $\mathrm{VF}(\mathrm{mV}) @ \mathrm{IF}=100 \mathrm{~mA}$ & 323.26 & 329.8 & .6 .54 & 3.15 & 1.3 & 1.85 \\
\hline$V F(m V) @ I F=1 A$ & 500.15 & 500.11 & 0.04 & 3.49 & 3.76 & -0.27 \\
\hline$V F(m V) @ I F=2 A$ & 650.35 & 642.08 & 8.27 & 5.24 & 7.23 & -1.99 \\
\hline $\mathrm{IR}(\mu \mathrm{A}) @ \mathrm{VR}=6 \mathrm{~V}$ & 1.51 & 1.01 & 0.5 & 0.3 & 0.12 & 0.17 \\
\hline $\mathrm{IR}(\mathrm{A}) @ \mathrm{VR}=36 \mathrm{~V}$ & 6.1 & 4.52 & 1.58 & 1.83 & 1.95 & -0.12 \\
\hline$V F(m V) @ I F=2 m A$ & 206.69 & 214.79 & .8 .1 & 4.26 & 2.83 & 1.43 \\
\hline $\mathrm{VF}(\mathrm{mV}) @ \mathrm{IF}=10 \mathrm{~mA}$ & 249.96 & 257.75 & -7.79 & 4.09 & 2.83 & 1.20 \\
\hline $\mathrm{VF}(\mathrm{mV}) @ \mathrm{IF}=100 \mathrm{~mA}$ & 322.18 & 329.27 & -7.09 & 3.98 & 2.87 & 1.11 \\
\hline $\mathrm{VF}(\mathrm{mV}) @ \mathrm{IF}=1 \mathrm{~A}$ & 494.17 & 499.01 & .4 .84 & 5.69 & 4.19 & 1.5 \\
\hline$V F(m V) @ I F=2 A$ & 637.79 & 640.16 & -2.37 & 9.41 & 6.14 & 3.27 \\
\hline
\end{tabular}




\begin{tabular}{|c|c|c|c|c|c|c|}
\hline \multirow[t]{2}{*}{ TEST } & \multicolumn{3}{|c|}{ MINIMUM VALUE } & \multicolumn{3}{|c|}{ MAXIMUM VALUE } \\
\hline & Freon & DI Water & Delta & Freon & Dl Water & Delta \\
\hline $\mathrm{ICBO}(\mathrm{nA}) @ \mathrm{VCB}=60 \mathrm{~V}$ & 0.49 & 0.45 & 0,04 & 3.2 & 5,8 & -2.6 \\
\hline $\mathrm{ICBO}(\mathrm{nA}) @ \mathrm{VCB}=75 \mathrm{~V}$ & 0.45 & 0.45 & 0 & 13 & 18 & -.5 \\
\hline $\mathrm{IEBO}(\mathrm{nA}) @ V E B=4 \mathrm{~V}$ & 0.01 & 0.01 & 0 & 1.7 & 0.01 & 1.69 \\
\hline $\operatorname{ICER}(\mathrm{nA}) @ \mathrm{VC}=50 \mathrm{~V}, \mathrm{R}=5 \mathrm{~K}$ & 0.32 & 0.27 & 0.05 & 2 & 5.4 & .3 .4 \\
\hline $\operatorname{ICES}(\mathrm{nA}) @ \mathrm{VCE}=.50 \mathrm{~V}$ & 0.15 & 0.11 & 0.04 & 1.8 & 5.3 & -3.5 \\
\hline $\mathrm{HFE} @ \mathrm{VCE}=10 \mathrm{~V}, \mathrm{IC}=0.1 \mathrm{~mA}$ & 138.4 & 163.5 & -25.1 & 191.6 & 182.8 & 8.8 \\
\hline $\mathrm{HFE} @ \mathrm{VCE}=10 \mathrm{~V}, \mathrm{IC}=1 \mathrm{~mA}$ & 161.3 & 167.8 & -6.5 & 198 & 183.5 & 14,5 \\
\hline $\mathrm{HFE} @ \mathrm{VCE}=10 \mathrm{~V}, \mathrm{IC}=10 \mathrm{~mA}$ & 182.1 & 169.8 & 12.3 & 207 & 187.6 & 19.4 \\
\hline $\mathrm{HFE} @ \mathrm{VCE}=10 \mathrm{~V}, \mathrm{IC}=150 \mathrm{~mA}$ & 180.3 & 167.4 & 12.9 & 210 & 186.6 & 23,4 \\
\hline $\mathrm{HFE} @ \mathrm{VCE}=10 \mathrm{~V}, \mathrm{IC}=500 \mathrm{~mA}$ & 96.3 & 93.9 & 2.4 & 115.6 & 104.4 & 11.2 \\
\hline $\mathrm{VCES}(\mathrm{mV}) @ \mathrm{IC}=150 \mathrm{~mA}, \mathrm{IB}=15 \mathrm{~mA}$ & 147 & 152 & -5 & 158 & 163.5 & .5 .5 \\
\hline VCES $(\mathrm{mV}) @ I \mathrm{C}=500 \mathrm{~mA}, \mathrm{IB}=50 \mathrm{~mA}$ & 363 & 368 & -5 & 386 & 391 & .5 \\
\hline $\begin{array}{l}\text { VBEON (mV) @ VCE }=10 \mathrm{~V}, \\
I \mathrm{C}=20 \mathrm{~mA}\end{array}$ & 705.5 & 716.5 & -11 & 712.5 & 720.5 & .8 \\
\hline VBEON $(\mathrm{mV}) @ \mathrm{VCE}=10 \mathrm{~V}, ;=50 \mathrm{n}: \mathrm{A}$ & 730 & 741 & -11 & 737.5 & 745 & -7.5 \\
\hline VBES $(\mathrm{mV}) @ 1 \mathrm{C}=150 \mathrm{~mA}, \mathrm{IB}=15 \mathrm{~mA}$ & 886.5 & 873 & -6.5 & 872 & 877.5 & -5.5 \\
\hline $\mathrm{VBES}(\mathrm{mV}) @ \mathrm{IC}=500 \mathrm{~mA}, \mathrm{IB}=50 \mathrm{~mA}$ & $1,081.5$ & 1089.5 & -8 & 1,099 & 1,101 & -2 \\
\hline $\mathrm{ICBO}(\mathrm{nA}) @ \mathrm{VCB}=.50 \mathrm{~V}$ & 0.74 & 0.65 & 0.09 & 0.83 & 0.68 & 0.15 \\
\hline $\mathrm{ICBO}(\mathrm{n} A) @ \mathrm{VCB}=-60 \mathrm{~V}$ & 0.6 & 0.55 & 0.05 & 0.8 & 0.7 & 0.1 \\
\hline $\operatorname{ICER}(\mathrm{nA}) @ V C=-50 \mathrm{~V}, \mathrm{R}=5 \mathrm{~K}$ & 0.44 & 0,36 & 0.08 & 0.52 & 0.4 & 0.12 \\
\hline IEBO (nA)@VEB $=-4 \mathrm{~V}$ & 0.48 & 0.46 & 0.02 & 0.56 & 0.54 & 0.02 \\
\hline $\mathrm{HFE} @ \mathrm{VCE}=-10 \mathrm{~V}, \mathrm{IC}=-0.1 \mathrm{~mA}$ & 97.7 & 103,1 & .5 .4 & 106.1 & 110.1 & -4 \\
\hline $\mathrm{HFE} @ \mathrm{VCE}=-10 \mathrm{~V}, \mathrm{IC}=-1 \mathrm{~mA}$ & 112.4 & 120.6 & -8.2 & 122.9 & 129 & -6.1 \\
\hline $\mathrm{HFE} @ \mathrm{VCE}=-10 \mathrm{~V}, \mathrm{IC}=-10 \mathrm{~mA}$ & 124.6 & 135.5 & -10.9 & 137.2 & 145.4 & -8.2 \\
\hline HFE @ VCE $=-10 \mathrm{~V}, \mathrm{IC}=-150 \mathrm{~mA}$ & 130.2 & 142 & -11.8 & 142 & 153.7 & .11 .7 \\
\hline HFE @ VCE $=-10 \mathrm{~V}, \mathrm{IC}=-500 \mathrm{~mA}$ & 81.3 & 90.7 & -9.4 & 93.3 & 102.5 & $-9,2$ \\
\hline VCES $(\mathrm{mV}) @ \mathrm{IC}=-150 \mathrm{~mA}, 1 \mathrm{~B}=-15 \mathrm{~mA}$ & 162 & 157 & 5 & 170.5 & 163.5 & 7 \\
\hline VCES $(\mathrm{mV}) @ I C=-500 \mathrm{~mA}, I \mathrm{~B}=-50 \mathrm{~mA}$ & 411 & 396 & 15 & 447.5 & 418.5 & 29 \\
\hline VBEON(mV)@VCE $=-10 \mathrm{~V}, I \mathrm{C}=-20 \mathrm{~mA}$ & 712.5 & 716 & -3.5 & 716.5 & 719.5 & .3 \\
\hline $\mathrm{VBEON}(\mathrm{mV}) @ \mathrm{VCE}=-10 \mathrm{~V}, \mathrm{IC}=.50 \mathrm{~mA}$ & 736 & 739 & -3 & 740.5 & 741.5 & -1 \\
\hline VBES $(\mathrm{mV}) @ 1 \mathrm{IC}=-150 \mathrm{~mA}, I \mathrm{IB}=.15 \mathrm{~mA}$ & 881 & 880.5 & 0.5 & 884.5 & 883.5 & 1 \\
\hline VBES $(\mathrm{mV}) @ \mathrm{IC}=-500 \mathrm{~mA}, \mathrm{IB}=-50 \mathrm{~mA}$ & $1,083.5$ & 1,073 & 10.5 & $1,094.5$ & 1.081 & 13.5 \\
\hline $\mathrm{IR}(\mu \mathrm{A}) @ \mathrm{VR}=6 \mathrm{~V}$ & 1.3 & 0.92 & 0.38 & 1.97 & 4.73 & -2.76 \\
\hline $\mathrm{IR}(\mu \mathrm{A}) @ \mathrm{VR}=36 \mathrm{~V}$ & 5.14 & 3.87 & 1.27 & 8.63 & 31.7 & -23.07 \\
\hline $\mathrm{VF}(\mathrm{mV}) @ \mathrm{IF}=2 \mathrm{~mA}$ & 200.5 & 211.5 & -11 & 209.5 & 217.5 & -8 \\
\hline $\mathrm{VF}(\mathrm{mV}) @ \mathrm{IF}=10 \mathrm{~m} \mathrm{~A}$ & 244 & 255 & .11 & 253.5 & 260 & -6.5 \\
\hline$V F(m V) @ I F=100 \mathrm{~mA}$ & 316.5 & 326 & -9.5 & 326 & 332 & -6 \\
\hline $\mathrm{VF}(\mathrm{mV}) @ \mathrm{IF}=1 \mathrm{~A}$ & 489 & 494.5 & -5.5 & 502.5 & 504 & -1.5 \\
\hline$V F(m V) @ I F=2 A$ & 633 & 633 & 0 & 652 & 648 & 4 \\
\hline $\mathrm{IR}(\mu \mathrm{A}) @ \mathrm{VR}=6 \mathrm{~V}$ & 1.25 & 0.9 & 0.35 & 2.44 & 1.08 & 1.36 \\
\hline $\mathrm{IR}(\mu \mathrm{A}) @ \mathrm{VR}=36 \mathrm{~V}$ & 5.11 & 3.84 & 1.27 & 15.6 & 5.87 & 9.73 \\
\hline $\mathrm{VF}(\mathrm{mV}) @ I F=2 \mathrm{~mA}$ & 198 & 212.5 & -14.5 & 211.5 & 217.5 & -6 \\
\hline $\mathrm{VF}(\mathrm{mV}) @ \mathrm{IF}=10 \mathrm{~mA}$ & 241.5 & 255.5 & -14 & 255 & 260.5 & -5.5 \\
\hline $\mathrm{VF}(\mathrm{mV}) @ \mathrm{IF}=100 \mathrm{~mA}$ & 314 & 327.5 & -13.5 & 327 & 332 & -5 \\
\hline$V F(m V) @ I F=1 \mathrm{~A}$ & 485 & 495.5 & -10.5 & 511 & 504.5 & 6.5 \\
\hline$V F(m V) @ I F=2 A$ & 626.5 & 633 & -6.5 & 674 & 649 & 25 \\
\hline
\end{tabular}




\begin{tabular}{|c|c|c|c|c|c|c|}
\hline \multirow[t]{2}{*}{$\overline{\text { TEST }}$} & \multicolumn{3}{|c|}{ AVERAGE } & \multicolumn{3}{|c|}{ STANDARD DEVIATION } \\
\hline & Freon & DI Water & Delta & Freon & DI Water & Delta \\
\hline $\mathrm{ICBO}(\mathrm{nA}) @ \mathrm{VCB}=60 \mathrm{~V}$ & 0.64 & 0,93 & .0 .29 & 0,61 & 1.3 & -0.69 \\
\hline $\mathrm{ICBO}(\mathrm{nA}) @ \mathrm{VCB}=75 \mathrm{~V}$ & 0.78 & 1.6 & -0.82 & 1.2 & 3.1 & $-1,9$ \\
\hline IEBO (nA)@ VEB =4V & 0.11 & 0.01 & 0.1 & 0.38 & 0 & 0.38 \\
\hline ICER (nA)@VC $=50 \mathrm{~V}, \mathrm{R}=5 \mathrm{~K}$ & 0.41 & 0.64 & -0.23 & 0.37 & 1.2 & -0.83 \\
\hline $\operatorname{ICES}(\mathrm{nA}) @ \mathrm{VCE}=.50 \mathrm{~V}$ & 0.25 & 0.51 & -0.26 & 0.37 & 1.2 & -0.83 \\
\hline HFE @ VCE $=10 \mathrm{~V}, I \mathrm{C}=0.1 \mathrm{~mA}$ & 176.91 & 174.87 & 2.04 & 15.38 & 4.74 & 10.61 \\
\hline $\mathrm{HFE} @ \mathrm{VCE}=10 \mathrm{~V}, \mathrm{IC}=1 \mathrm{~mA}$ & 186.59 & 174.9 & 11.69 & 10,52 & 4.52 & 6 \\
\hline $\mathrm{HFE} @ \mathrm{VCE}=10 \mathrm{~V}, \mathrm{IC}=10 \mathrm{~mA}$ & 198.15 & 178.03 & 20.12 & 9.23 & 4.92 & 4.3 \\
\hline HFE@VCE $=10 \mathrm{~V}, \mathrm{IC}=150 \mathrm{~mA}$ & 199.75 & 176.91 & 22.84 & 9,95 & 5.2 & 4.75 \\
\hline $\mathrm{HFE} @ \mathrm{VCE}=10 \mathrm{~V}, \mathrm{IC}=500 \mathrm{~mA}$ & 107.32 & 98.86 & 8.46 & 5.5 & 3.05 & 2.44 \\
\hline VCES $(\mathrm{mV})(0) \mathrm{IC}=150 \mathrm{~mA}, I \mathrm{IB}=15 \mathrm{~mA}$ & 152.25 & 158.03 & $-5,78$ & 3.01 & 3.72 & -0.71 \\
\hline VCES $(\mathrm{mV}) @ 1 \mathrm{IC}=500 \mathrm{~mA}, \mathrm{IB}=50 \mathrm{~mA}$ & 378.75 & 380.73 & .1 .98 & 5.29 & 6.71 & -1.42 \\
\hline VBEON $(\mathrm{mV}) @ \mathrm{VCE}=10 \mathrm{~V}, \mathrm{IC}=20 \mathrm{~mA}$ & 708.68 & 718.78 & -10.1 & 2.01 & 0.88 & 1.13 \\
\hline VBEON(mV)@VCE $=10 \mathrm{~V}, \mathrm{IC}=50 \mathrm{~mA}$ & 733.35 & 743.33 & -9.98 & 2.05 & 1.04 & 1.01 \\
\hline VBES(mV)@IC $=150 \mathrm{~mA}, \mathrm{IB}=15 \mathrm{~mA}$ & 869.55 & 875.5 & .5 .95 & 1.63 & 1.32 & 0.31 \\
\hline VBES $(\mathrm{mV}) @ I C=500 \mathrm{~mA}, \mathrm{IB}=50 \mathrm{~mA}$ & 1.092 .1 & $1,095,2$ & -3.1 & 4.42 & 3.55 & 0.87 \\
\hline$I C B O(n A) @ V C B=.50 V$ & 0.75 & 0.66 & 0.09 & 0.02 & 0.01 & 0.01 \\
\hline $\mathrm{ICBO}(\mathrm{nA}) @ \mathrm{VCB}=-60 \mathrm{~V}$ & 0.67 & 0.59 & 0.08 & 0.05 & 0.04 & 0.01 \\
\hline $\operatorname{ICER}(\mathrm{nA}) @ V C=-50 \mathrm{~V}, \mathrm{R}=5 \mathrm{~K}$ & 0.45 & 0.37 & 0,08 & 0,02 & 0.01 & 0.01 \\
\hline IEBO (nA)@VEB $=-4 V$ & 0.52 & 0.49 & 0.03 & 0.02 & 0.02 & 0 \\
\hline HFE@VCE $=-10 \mathrm{~V}, \mathrm{IC}=-0.1 \mathrm{~mA}$ & 102.97 & 107.98 & $-5,01$ & 2.09 & 1.61 & 0.48 \\
\hline HFE @ VCE $=-10 \mathrm{~V}, \mathrm{IC}=-1 \mathrm{~mA}$ & 118.62 & 126.37 & -7.75 & 2.59 & 2 & 0.59 \\
\hline HFE (Q) VCE $=-10 \mathrm{~V}, I C=-10 \mathrm{~mA}$ & 131.96 & 142.03 & -10.07 & 3.13 & 2,4 & 0.73 \\
\hline $\mathrm{HFE} @ \mathrm{VCE}=-10 \mathrm{~V}, \mathrm{IC}=-150 \mathrm{~mA}$ & 137.39 & 148.13 & -10.74 & 3.66 & 3.37 & 0.29 \\
\hline HFE @ VCE $=-10 \mathrm{~V}, \mathrm{IC}=-500 \mathrm{~mA}$ & 89.63 & 98.93 & -9.31 & 3,17 & 2.85 & 0.33 \\
\hline $\mathrm{VCES}(\mathrm{mV}) @ \mathrm{IC}=-150 \mathrm{~mA}, \mathrm{IB}=-15 \mathrm{~mA}$ & 165 & 159.18 & 5.82 & 1,98 & 1.76 & 0.22 \\
\hline VCES(mV)@ $I C=-500 \mathrm{~mA}, I B=-50 \mathrm{~mA}$ & 423.33 & 403.08 & 20.25 & 8.89 & 5.95 & 2.94 \\
\hline VBEON $(\mathrm{mV}) @ V C E=-10 \mathrm{~V}, \mathrm{IC}=-20 \mathrm{~mA}$ & 714.68 & 717.58 & -2.9 & $0.9 !$ & 0.85 & 0.06 \\
\hline VBEON $(\mathrm{mV}) @ V C E=-10 \mathrm{~V}, \mathrm{IC}=-50 \mathrm{~mA}$ & 738.28 & 740.25 & -1.97 & 1.03 & 0.73 & 0.3 \\
\hline VBES(mV) @IC $=-150 \mathrm{~mA}, \mathrm{IB}=-15 \mathrm{~mA}$ & 882.25 & 882.18 & 0.07 & 0.98 & 0.77 & 0.21 \\
\hline VBES $(m \mathrm{~m}) @ \mathrm{IC}=-500 \mathrm{~mA}, \mathrm{IB}=-50 \mathrm{~mA}$ & $1,087.9$ & $1,077.7$ & 10.2 & 3,05 & 2.2 & 0.85 \\
\hline $\operatorname{IR}(\mu \mathrm{A}) @ V R=6 \mathrm{~V}$ & 1.61 & 1.18 & 0.43 & 0.16 & 0.84 & -0.67 \\
\hline$I R(\mu A) @ V R=36 V$ & 6.28 & 5.49 & 0.79 & 0.76 & 6.17 & $-5,41$ \\
\hline $\mathrm{VF}(\mathrm{mIV}) @ \mathrm{IF}=2 \mathrm{~mA}$ & 205.13 & 215.2 & -10.07 & 2.3 & 1.41 & 0.89 \\
\hline $\mathrm{VF}(\mathrm{mV}) @ \mathrm{IF}=10 \mathrm{~mA}$ & 248.38 & 258.13 & -9.75 & 2.29 & 1.49 & 0.8 \\
\hline $\mathrm{VF}(\mathrm{mV}) @ \mathrm{IF}=100 \mathrm{~mA}$ & 321.23 & 329.6 & -8.37 & 2.31 & 1.5 & 0.81 \\
\hline $\mathrm{VF}(\mathrm{mV}) @ \mathrm{IF}=1 \mathrm{~A}$ & 496.73 & 499.05 & -2.32 & 3.48 & 2.7 & 0.78 \\
\hline $\mathrm{VF}(\mathrm{mV}) @ \mathrm{IF}=2 \mathrm{~A}$ & 644.28 & 639.75 & 4.53 & 5.66 & 4.32 & 1.34 \\
\hline $\mathrm{IR}(\mu \mathrm{A}) @ \mathrm{VR}=6 \mathrm{~V}$ & 1.68 & 0.99 & 0.69 & 0.27 & 0.05 & 0.23 \\
\hline $\mathrm{IR}(\mu \mathrm{A}) @ \mathrm{VR}=36 \mathrm{~V}$ & 7.04 & 4.35 & 2.69 & 2.8 & 0.41 & 2.39 \\
\hline $\mathrm{VF}(\mathrm{mV}) @ \mathrm{IF}=2 \mathrm{~mA}$ & 204.25 & 215.5 & -11.25 & 3.23 & 1.18 & 2.05 \\
\hline $\mathrm{VF}(\mathrm{mV}) @ \mathrm{IF}=10 \mathrm{~mA}$ & 247.68 & 258.4 & -10.72 & 3.11 & 1,33 & 1.78 \\
\hline $\mathrm{VF}(\mathrm{mV}) @ \mathrm{IF}=100 \mathrm{~mA}$ & 320.2 & 329.95 & -9.75 & 3.01 & 1.29 & 1.72 \\
\hline $\mathrm{VF}(\mathrm{mV}) @ \mathrm{IF}=1 \mathrm{~A}$ & 493.58 & 499.85 & -6.27 & 5.34 & 2.52 & 2.82 \\
\hline $\mathrm{VF}(\mathrm{mV}) @ \mathrm{IF}=2 \mathrm{~A}$ & 638.68 & 641.03 & -2.35 & 10.31 & 4.55 & 5.76 \\
\hline
\end{tabular}




\begin{tabular}{|c|c|c|c|c|c|c|}
\hline TEST & MI & IMUM VAL & & $\overline{M A}$ & IMUM VAI & \\
\hline & Freon & DI Water & Delta & Freon & DI Water & Delta \\
\hline $\mathrm{ICBO}(\mathrm{nA}) @ \mathrm{VCB}=60 \mathrm{~V}$ & 0.46 & 0.43 & 0.03 & 0.59 & 3.5 & -2.91 \\
\hline $\mathrm{ICBO}(\mathrm{nA}) @ V \mathrm{CB}=75 \mathrm{~V}$ & $0 . \overline{45}$ & 0,35 & 0.1 & 0.65 & 14 & .13 .35 \\
\hline $\operatorname{IEBO}(\mathrm{nA}) @ \mathrm{VEB}=4 \mathrm{~V}$ & 0.01 & 0.01 & 0 & 9.2 & 0.13 & 9.07 \\
\hline $\operatorname{ICER}(\mathrm{nA}) @ \mathrm{VC}=50 \mathrm{~V}, \mathrm{R}=5 \mathrm{~K}$ & 0.32 & 0.25 & 0.07 & 0.42 & 0.87 & -0.45 \\
\hline $\operatorname{ICES}(\mathrm{nA}) @ \mathrm{VCE}=-50 \mathrm{~V}$ & 0.16 & 0.11 & 0.05 & 0.25 & 0.74 & -0.49 \\
\hline $\mathrm{HFE} @ \mathrm{VCE}=10 \mathrm{~V}, \mathrm{IC}=0,1 \mathrm{~mA}$ & 145.8 & 165.7 & -19.9 & 190.1 & 178.7 & 11.4 \\
\hline $\mathrm{HFE} @ \mathrm{VCE}=10 \mathrm{~V}, \mathrm{IC}=1 \mathrm{~mA}$ & 167.5 & 165.6 & 1.9 & 196.8 & 178.3 & 18.5 \\
\hline HFE @ VCE $=10 \mathrm{~V}, \mathrm{IC}=10 \mathrm{~mA}$ & 175.6 & 167.8 & 7.8 & 207 & 182.1 & 24.9 \\
\hline HFE @ VCE $=10 \mathrm{~V}, I \mathrm{C}=150 \mathrm{~mA}$ & 174.4 & 165.9 & 8.5 & 210 & 180.3 & 29.7 \\
\hline $\mathrm{HFE} @ \mathrm{VCE}=10 \mathrm{~V}, \mathrm{IC}=500 \mathrm{~mA}$ & 95.6 & 93.6 & 2.3 & 114.8 & 102.7 & 12.1 \\
\hline $\operatorname{VCES}(\mathrm{mV}) @ \mathrm{IC}=150 \mathrm{~mA}, \mathrm{IB}=15 \mathrm{~mA}$ & 147.5 & 151.5 & -4 & 160.5 & 164.5 & -4 \\
\hline VCES(mV)@IC $=500 \mathrm{~mA}, 1 \mathrm{~B}=50 \mathrm{~mA}$ & 371.5 & 367.5 & 4 & 390 & 393.5 & -3.5 \\
\hline VBEON $(\mathrm{mV}) @ \mathrm{VCE}=10 \mathrm{~V}, \mathrm{IC}=20 \mathrm{~mA}$ & 706.5 & 719.5 & -13 & 717.5 & 722.5 & -5 \\
\hline $\mathrm{VBEON}(\mathrm{mV}) @ \mathrm{VCE}=10 \mathrm{~V}, \mathrm{IC}=50 \mathrm{~mA}$ & 732 & 743 & -11 & 742.5 & 747 & -4.5 \\
\hline $\mathrm{VBES}(\mathrm{mV}) @ \mathrm{IC}=150 \mathrm{~mA}, \mathrm{IB}=15 \mathrm{~mA}$ & 868 & 874 & -6 & 874.5 & 879 & -4.5 \\
\hline VBES $(\mathrm{mV}) @ I C=500 \mathrm{~mA}, I \mathrm{IB}=50 \mathrm{~mA}$ & 1,088 & $1,089,5$ & -1.5 & 1,102 & $1,102.5$ & -0.5 \\
\hline $\mathrm{ICBO}(\mathrm{nA}) @ \mathrm{VCB}=-50 \mathrm{~V}$ & 0.7 & 0.64 & 0.06 & 0.74 & 0.66 & 0.08 \\
\hline $1 \mathrm{CBO}(\mathrm{nA}) @ \mathrm{VCB}=-60 \mathrm{~V}$ & 0.6 & 0.55 & 0.05 & 0.7 & 0.6 & 0.1 \\
\hline $\operatorname{ICER}(\mathrm{nA}) @ \mathrm{VC}=-50 \mathrm{~V}, \mathrm{R}=5 \mathrm{~K}$ & 0.4 & 0.36 & 0.04 & $0 . \overline{46}$ & 0.4 & 0.06 \\
\hline IEBO $(\mathrm{nA}) @ \mathrm{VEB}=-4 \mathrm{~V}$ & 0.43 & 0.45 & -0.02 & 0.56 & 0.53 & 0.03 \\
\hline HFE @ VCE $=-10 \mathrm{~V}, \mathrm{IC}=-0.1 \mathrm{~mA}$ & 100.7 & 101.6 & .0 .9 & 105.7 & 109.4 & -3.7 \\
\hline $\mathrm{HFE} @ \mathrm{VCE}=-10 \mathrm{~V}, \mathrm{IC}=-1 \mathrm{~mA}$ & 116.1 & 119.1 & .3 & 122.9 & 128.5 & -5.6 \\
\hline HFE @ VCE $=-10 \mathrm{~V}, I \mathrm{C}=-10 \mathrm{~mA}$ & 128.9 & 133.9 & .5 & 137.6 & 144.9 & -7.3 \\
\hline HFE @ VCE $=-10 \mathrm{~V}, \mathrm{IC}=-150 \mathrm{~mA}$ & 133.9 & 139.9 & -6 & 144.2 & 153.7 & -9.5 \\
\hline HFE@VCE $=-10 \mathrm{~V}, \mathrm{IC}=-500 \mathrm{~mA}$ & 82.6 & 89.03 & -6.43 & 93.3 & 102.5 & -9.2 \\
\hline VCES(mV)@IC $=-150 \mathrm{~mA}, \mathrm{IB}=-15 \mathrm{~mA}$ & 162.5 & 156 & 6.5 & 168 & 165.5 & 2.5 \\
\hline VCES $(\mathrm{mV}) @ I C=-500 \mathrm{~mA}, I \mathrm{~B}=-50 \mathrm{~mA}$ & 415 & 392.5 & 22.5 & 441.5 & 425 & 16.5 \\
\hline VBEON(mV)@VCE $=-10 \mathrm{~V}, \mathrm{IC}=-20 \mathrm{~mA}$ & 714.5 & 718 & -3.5 & 721 & 721.5 & -0.5 \\
\hline VBEON(mV)@VCE $=-10 \mathrm{~V}, \mathrm{IC}=-50 \mathrm{mLA}$ & 738 & 740 & -2 & 744 & 744 & 0 \\
\hline VBES(mV)@IC $=-150 \mathrm{~mA}, I \mathrm{~B}=-15 \mathrm{~mA}$ & 881.5 & 881.5 & 0 & 888 & 885 & 3 \\
\hline VBES $(\mathrm{mV}) @ \mathrm{IC}=-500 \mathrm{~mA}, \mathrm{IB}=-50 \mathrm{~mA}$ & 1,083 & 1073 & 10 & $1,095.5$ & 1,084 & 11.5 \\
\hline IR $(\mu \mathrm{A}) @ \mathrm{VR}=6 \mathrm{~V}$. & 0.98 & 0.83 & 0.15 & 2.04 & 1 & 1.04 \\
\hline $\mathrm{IR}(\mu \mathrm{A}) @ \mathrm{VR}=36 \mathrm{~V}$ & 4.09 & 3.35 & 0.74 & 15.6 & 4.07 & 11.53 \\
\hline $\mathrm{VF}(\mathrm{mV}) @ \mathrm{IF}=2 \mathrm{~mA}$ & 199.5 & 214 & -14.5 & 216 & 218 & -2 \\
\hline $\mathrm{VF}(\mathrm{mV}) @ \mathrm{IF}=10 \mathrm{~mA}$ & 243.5 & 257 & -13.5 & 259 & 261 & -2 \\
\hline $\mathrm{VF}(\mathrm{mV}) @ \mathrm{IF}=100 \mathrm{~mA}$ & 316.5 & 328.5 & -12 & 331 & 332.5 & -1.5 \\
\hline$V F(m V) @ I F=1 \mathrm{~A}$ & 491.5 & 496 & -4.5 & 512 & 505 & 7 \\
\hline $\mathrm{VF}(\mathrm{mV}) @ \mathrm{IF}=2 \mathrm{~A}$ & 633 & 633.5 & -0.5 & 671 & 649.5 & 21.5 \\
\hline $\mathrm{IR}(\mu \mathrm{A}) @ \mathrm{VR}=6 \mathrm{~V}$ & 1.12 & 0,82 & 0.3 & 2.63 & 1.18 & 1.45 \\
\hline $\mathrm{IR}(\mu \mathrm{A}) @ \mathrm{VR}=36 \mathrm{~V}$ & 4.45 & 3.58 & 0.87 & 12.8 & $5 . \overline{46}$ & 7.34 \\
\hline $\mathrm{VF}(\mathrm{mV}) @ \mathrm{IF}=2 \mathrm{~mA}$ & 192.5 & 210 & -17.5 & 213 & 219.5 & -6.5 \\
\hline $\mathrm{VF}(\mathrm{mV}) @ \mathrm{IF}=10 \mathrm{~mA}$ & 236 & 252.5 & -16.5 & 256 & 262 & -6 \\
\hline $\mathrm{VF}(\mathrm{mV}) @ \mathrm{IF}=100 \mathrm{~mA}$ & 308.5 & 324 & -15.5 & 328.5 & 333.5 & -5 \\
\hline $\mathrm{VF}(\mathrm{mV}) @ \mathrm{IF}=1 \mathrm{~A}$ & 479 & 490 & -11 & 501 & 506 & -5 \\
\hline$V F(m V) @ I F=2 A$ & 621.5 & 62.7 & -5.5 & 646 & 651 & -5 \\
\hline
\end{tabular}




\begin{tabular}{|c|c|c|c|c|c|c|}
\hline \multirow[t]{2}{*}{ TEST } & \multicolumn{3}{|c|}{ AVERAGE } & \multicolumn{3}{|c|}{ STANDARD DEVIATION } \\
\hline & Freon & DI Water & Delta & Freon & DI Water & Delta \\
\hline$I C B O(n A) @ V C B=60 V$ & 0.51 & 0,64 & -0.13 & 0.03 & 0.64 & -0.61 \\
\hline $\mathrm{ICBO}(\mathrm{nA}) @ V C B=75 \mathrm{~V}$ & 0.53 & 1.3 & -0.77 & 0.04 & 2.9 & -2.86 \\
\hline $\operatorname{IEBO}(\mathrm{nA}) @ \mathrm{VEB}=4 \mathrm{~V}$ & 0.61 & 0.02 & 0.59 & 2.1 & 0.04 & 2.06 \\
\hline $\operatorname{ICER}(\mathrm{nA}) @ \mathrm{VC}=50 \mathrm{~V}, \mathrm{R}=5 \mathrm{~K}$ & 0.35 & 0.33 & 0.02 & 0.02 & 0.12 & -0.1 \\
\hline $\operatorname{ICES}(\mathrm{nA}) @ \mathrm{VCE}=.50 \mathrm{~V}$ & 0.19 & 0.2 & -0.01 & 0.02 & 0.13 & -0.11 \\
\hline HFE $@ V C E=10 \mathrm{~V}, I C=0.1 \mathrm{~mA}$ & 176.74 & 172.78 & 3.96 & 10.99 & 4.36 & 6.63 \\
\hline $\mathrm{HFE} @ \mathrm{VCE}=10 \mathrm{~V}, \mathrm{IC}=1 \mathrm{~mA}$ & 183.71 & 171.99 & 11.72 & 9.14 & 4.3 & 4.88 \\
\hline $\mathrm{HFE} @ \mathrm{VCE}=10 \mathrm{~V}, \mathrm{IC}=10 \mathrm{~mA}$ & 192.79 & 174.71 & 18.08 & 11.59 & 4.46 & 7.13 \\
\hline $\mathrm{HFE} @ \mathrm{~V} C \mathrm{E}=10 \mathrm{~V}, \mathrm{IC}=150 \mathrm{~mA}$ & 193.64 & 173.55 & 20.09 & 13.2 & 4.71 & 8.49 \\
\hline $\mathrm{HFE} @ \mathrm{VCE}=10 \mathrm{~V}, \mathrm{IC}=500 \mathrm{~mA}$ & 104.55 & 98.66 & 5.89 & 6.4 & 2.94 & 3.46 \\
\hline $\mathrm{VCES}(\mathrm{mV}) @ \mathrm{IC}=150 \mathrm{~mA}, \mathrm{IB}=15 \mathrm{~mA}$ & 153.93 & 159.59 & -5.66 & 4.03 & 3.48 & 0.55 \\
\hline VCES(mV)@IC $=500 \mathrm{~mA}, I \mathrm{~B}=50 \mathrm{~mA}$ & 380,95 & 383.66 & .2 .71 & 5.28 & 6.75 & -1.47 \\
\hline $\mathrm{VBE} O \mathrm{~N}(\mathrm{mV}) @ \mathrm{VCE}=10 \mathrm{~V}, \mathrm{IC}=20 \mathrm{~mA}$ & 710.68 & 720.64 & 9,96 & 3.04 & 0.93 & 2.11 \\
\hline VBEON(mV)@VCE $=10 \mathrm{~V}, \mathrm{IC}=50 \mathrm{~mA}$ & 735.63 & 745.02 & -9.39 & 3.04 & 1.01 & 2.03 \\
\hline $\mathrm{VBES}(\mathrm{mV}) @ \mathrm{IC}=150 \mathrm{~mA}, \mathrm{IB}=15 \mathrm{~mA}$ & 871.35 & 877.2 & -5.85 & 2.15 & 1.21 & 0.94 \\
\hline VBES(mV)@IC $=500 \mathrm{~mA}, \mathrm{IB}=50 \mathrm{~mA}$ & $1,094.8$ & $1,097.2$ & -2.4 & 3.62 & 3.06 & 0.56 \\
\hline$I C B O(n A) @ V C B=-50 V$ & 0.72 & 0.64 & 0.08 & 0.01 & 0.01 & 0 \\
\hline$I C B O(n A) @ V C B=-60 V$ & 0.65 & 0.6 & 0.05 & 0.05 & 0.02 & 0.03 \\
\hline$I C E R(n A) @ V C=-50 V, R=5 K$ & 0.43 & 0.37 & 0.06 & 0.02 & 0.01 & 0.01 \\
\hline IEBO $(n A) @ V E B=-4 V$ & 0.49 & 0.48 & 0.01 & 0.04 & 0.02 & 0.02 \\
\hline HFE @ VCE $=-10 \mathrm{~V}, \mathrm{IC}=-0.1 \mathrm{~mA}$ & 103.16 & 106.15 & -2.99 & 1.55 & 1.97 & -0.42 \\
\hline HFE @ VCE $=-10 \mathrm{~V}, \mathrm{IC}=-1 \mathrm{~mA}$ & 119 & 124.51 & .5 .51 & 2.04 & 2.36 & -0.32 \\
\hline HFE @ VCE $=-10 \mathrm{~V}, \mathrm{IC}=-10 \mathrm{~mA}$ & 132.45 & 140.04 & .7 .59 & 2.69 & 2.73 & -0.04 \\
\hline $\mathrm{HFE} @ \mathrm{VCE}=-10 \mathrm{~V}, \mathrm{IC}=-150 \mathrm{~mA}$ & 138.53 & 146.36 & .7 .83 & 3.02 & 3.05 & -0.03 \\
\hline HFE @VCE $=-10 \mathrm{~V}, \mathrm{IC}=-500 \mathrm{~mA}$ & 89.65 & 97.89 & -8.24 & 3.55 & 3.45 & 0.1 \\
\hline $\mathrm{VCES}(\mathrm{mV}) @) \mathrm{IC}=-150 \mathrm{~mA}, \mathrm{IB}=-15 \mathrm{~mA}$ & 164.53 & 159.09 & 5.44 & 1.7 & 2.5 & -0.8 \\
\hline $\operatorname{VCES}(\mathrm{mV}) @ \mathrm{IC}=-500 \mathrm{~mA}, I \mathrm{~B}=-50 \mathrm{~mA}$ & 423.15 & 403.16 & 19.99 & 8.47 & 7.96 & -0.51 \\
\hline VBEON $(m V) @ V C E=-10 V, I C=-20 \mathrm{~mA}$ & 716.6 & 719.55 & -2.95 & 1.68 & 0.9 & 0.78 \\
\hline VBEON(mV)@VCE $=-10 \mathrm{~V}, I \mathrm{C}=-50 \mathrm{~mA}$ & 740.08 & 742.02 & -1.94 & 1.6 & 1.03 & 0.57 \\
\hline VBES $(\mathrm{mV})$ (2) $I \mathrm{C}=-150 \mathrm{~mA}, \mathrm{IB}=-15 \mathrm{~mA}$ & 883.88 & 883.36 & 0.52 & 1.37 & 0.9 & 0.47 \\
\hline VBES $(\mathrm{mV})(0 \mathrm{IC}=-500 \mathrm{~mA}, \mathrm{IB}=-50 \mathrm{~mA}$ & $1,089.2$ & $1,078.1$ & 11.1 & 2.78 & 2.4 & 0.38 \\
\hline $\operatorname{IR}(\mu \mathrm{A}) @ \mathrm{~V} R=6 \mathrm{~V}$ & 1.48 & 0.9 & 0.58 & 0.27 & 0.04 & 0.23 \\
\hline $\mathrm{IR}(\mu \mathrm{A}) @ \mathrm{~V}=36 \mathrm{~V}$ & 6.43 & 3.78 & 2.65 & 2.57 & 0.2 & 2.37 \\
\hline $\mathrm{VF}(\mathrm{mV}) \otimes \mathrm{F}=2 \mathrm{~mA}$ & 206.85 & 216.64 & -9.79 & 4.14 & 1.1 & 3.04 \\
\hline $\mathrm{VF}(\mathrm{mV}) @ I F=10 \mathrm{~mA}$ & 250.2 & 259.34 & -9.14 & 3.88 & 1.06 & 2.82 \\
\hline $\mathrm{VF}(\mathrm{mV})(\mathrm{S})[\mathrm{F}=100 \mathrm{~mA}$ & 322.8 & 330.75 & -7.95 & 3.83 & 1.18 & 2.65 \\
\hline $\mathrm{VF}(\mathrm{mV}) @ \mathrm{IF}=1 \mathrm{~A}$ & 498.5 & 499.57 & -1.07 & 5.51 & 2.33 & 3.18 \\
\hline$V F(m V) @[F=2 A$ & 646.48 & 639.86 & 6.62 & 9.08 & 4.07 & 5.01 \\
\hline IR $(\mu \mathrm{A}) @ V R=6 \mathrm{~V}$ & 1.43 & 0.94 & 0.49 & 0.31 & 0.1 & 0.21 \\
\hline $\mathrm{IR}(\mu \mathrm{A}) @ \mathrm{VR}=36 \mathrm{~V}$ & 5.64 & 4.02 & 1.62 & 1.76 & 0.4 & 1.36 \\
\hline $\mathrm{VF}(\mathrm{mV}) @ \mathrm{IF}=2 \mathrm{~mA}$ & 207.5 & 215.98 & -8.48 & 4.21 & 2.62 & 1.59 \\
\hline $\mathrm{VF}(\mathrm{mV}) @ \mathrm{IF}=10 \mathrm{~mA}$ & 250.65 & 258.73 & -8.08 & 4.18 & 2.62 & 1.56 \\
\hline $\mathrm{VF}(\mathrm{mV}) @ \mathrm{IF}=100 \mathrm{~mA}$ & 322.75 & 330.2 & -7.45 & 4.13 & 2.71 & 1.42 \\
\hline $\mathrm{VF}(\mathrm{mV}) @ \mathrm{IF}=1 \mathrm{~A}$ & 493.85 & 500.36 & -6.51 & 4.88 & 4.27 & 0.61 \\
\hline$V F(m V) @ I F=2 A$ & 636.45 & 641.93 & -5.48 & 6.43 & 6.32 & 0.11 \\
\hline
\end{tabular}




\begin{tabular}{|c|c|c|c|c|c|c|}
\hline \multirow[t]{2}{*}{ TEST } & \multicolumn{3}{|c|}{ MINIMUM VALUE } & \multicolumn{3}{|c|}{ MAXIMUM VALUE } \\
\hline & Freon & DI Water & Delta & Freon & DI Water & Delta \\
\hline HFE @ VCE $-10 \mathrm{~V}, \mathrm{IC}=10 \mathrm{~mA}$ & 93.3 & 90.6 & 2.7 & 111.6 & 102.5 & 9.1 \\
\hline $\mathrm{HFE} @ \mathrm{VCE}=10 \mathrm{~V}, \mathrm{IC}=20 \mathrm{~mA}$ & 91.7 & 89.1 & 2.6 & 111.1 & 101.1 & 10 \\
\hline HFE @ VCE $=10 \mathrm{~V}, I C=50 \mathrm{~mA}$ & 91.2 & 88.3 & 2.9 & 111.6 & 101.1 & 10.5 \\
\hline VBEON $(\mathrm{mV}) @ \mathrm{VCE}=10 \mathrm{~V}, \mathrm{IC}=20 \mathrm{~mA}$ & 852.5 & 854.5 & -2 & 862 & 863 & -1 \\
\hline VBEON $(\mathrm{mV}) @ \mathrm{VCE}=-10 \mathrm{~V}, \mathrm{IC}=-50 \mathrm{~mA}$ & 870.5 & 872.5 & -2 & 880.5 & 881.5 & -1 \\
\hline $\mathrm{HFE} @ \mathrm{VCE}=-10 \mathrm{~V}, \mathrm{IC}=-\operatorname{ImA}$ & 58.6 & 64.3 & -5.7 & 65.4 & 69.8 & -4.4 \\
\hline $\mathrm{HFE} @ \mathrm{Q}, \mathrm{VCE}=-10 \mathrm{~V}, \mathrm{IC}=-20 \mathrm{~mA}$ & 67.3 & 74.5 & -7.2 & 75.8 & 81.5 & -5.8 \\
\hline $\mathrm{HFE} @ \mathrm{VCE}=-10 \mathrm{~V}, \mathrm{IC}=-50 \mathrm{~mA}$ & 71.7 & 79.4 & -7.7 & 81 & 86.8 & -5.8 \\
\hline VBEON(mV)@VCE $=-10 \mathrm{~V}, \mathrm{IC}=-20 \mathrm{~mA}$ & 853.5 & 851 & 2.5 & 862.5 & 859 & 3.5 \\
\hline VBEON (mV)@VCE $=-10 \mathrm{~V}, \mathrm{IC}=-50 \mathrm{~mA}$ & 871 & 868 & 3 & 880 & 875.5 & 4.5 \\
\hline $\mathrm{VF}(\mathrm{mV}) @ \mathrm{IF}=2 \mathrm{~mA}$ & 332 & 341 & -9 & 351.5 & 349 & 2.5 \\
\hline $\mathrm{VF}(\mathrm{mV}) @ \mathrm{IF}=10 \mathrm{~mA}$ & 366 & 375 & -9 & 384.5 & 383.5 & 1 \\
\hline $\mathrm{VF}(\mathrm{mV}) @ \mathrm{IF}=100 \mathrm{~mA}$ & 420 & 429.5 & -9.5 & 438.5 & 437 & 1.5 \\
\hline $\mathrm{VF}(\mathrm{mV}) @ \mathrm{IF}=1 \mathrm{~A}$ & 536.5 & 543 & .6 .5 & 555 & 552 & 3 \\
\hline$V F(m V) @ I F=2 A$ & 629 & 631 & -2 & 653.5 & 645.5 & 8 \\
\hline $\mathrm{VF}(\mathrm{mV}) @ \mathrm{IF}=2 \mathrm{~mA}$ & 326 & 335 & -9 & 348.5 & 348.5 & 0 \\
\hline $\mathrm{VF}(\mathrm{mV}) @ \mathrm{IF}=10 \mathrm{rnA}$ & 359.5 & 368 & -8.5 & 382 & 383 & -1 \\
\hline $\mathrm{VF}(\mathrm{mV}) @ \mathrm{IF}=100 \mathrm{~mA}$ & 413 & 422 & .9 & 436.5 & 437 & -0.5 \\
\hline $\mathrm{VF}(\mathrm{mV}) @ \mathrm{IF}=1 \mathrm{~A}$ & 528 & 534 & -6 & 553 & 554.5 & -1.5 \\
\hline $\mathrm{VF}(\mathrm{mV}) @ \mathrm{IF}=2 \mathrm{~A}$ & 618 & 622.5 & -4.5 & 647 & 649.5 & -2.5 \\
\hline
\end{tabular}

\begin{tabular}{|c|c|c|c|c|c|c|}
\hline \multirow[t]{2}{*}{ TEST } & \multicolumn{3}{|c|}{ AVERAGE VALUE } & \multicolumn{3}{|c|}{ STANDARD DEVIATION } \\
\hline & Freon & DI Water & Delta & Freon & DI Water & Delta \\
\hline $\mathrm{HFE} @ \mathrm{VCE}=10 \mathrm{~V}, \mathrm{IC}=10 \mathrm{~mA}$ & 104.63 & 95.52 & 9.11 & 5.83 & 2.85 & 2.98 \\
\hline HFE @ VCE $=10 \mathrm{~V}, \mathrm{IC}=20 \mathrm{~mA}$ & 103.56 & 94.25 & 9.31 & 5.83 & 2.82 & 3.01 \\
\hline $\mathrm{HFE} @ \mathrm{VCE}=10 \mathrm{~V}, \mathrm{IC}=50 \mathrm{~mA}$ & 103.81 & 93.49 & 10.32 & 6.18 & 2.84 & 3.34 \\
\hline VBEON $(\mathrm{mV}) @ \mathrm{VCE}=10 \mathrm{~V}, \mathrm{IC}=20 \mathrm{~mA}$ & 856.78 & 857.99 & -1.21 & 2.17 & 1.76 & 0.41 \\
\hline VBEON $(\mathrm{mV}) @ V C E=-10 \mathrm{~V}, \mathrm{IC}=-50 \mathrm{~mA}$ & 875.35 & 876.67 & -1.32 & 2.22 & 1.72 & 0.5 \\
\hline HFE @ VCE $=-10 \mathrm{~V}, I \mathrm{C}=-1 \mathrm{~mA}$ & 62.59 & 67.57 & -4.98 & 1.31 & 1.2 & 0.11 \\
\hline HFE @ VCE $=-10 \mathrm{~V}, \mathrm{IC}=-20 \mathrm{~mA}$ & 72.15 & 78.58 & -6.43 & 1.64 & 1.55 & 0.09 \\
\hline HFE @ VCE $=-10 \mathrm{~V}, \mathrm{IC}=-50 \mathrm{~mA}$ & 76.81 & 83.87 & -7.06 & 1.73 & 1.64 & 0.09 \\
\hline VBEON (mV)@VCE $=-10 \mathrm{~V}, \mathrm{IC}=-20 \mathrm{~mA}$ & 857.14 & 853.9 & 3.24 & 1.85 & 1.73 & 0.12 \\
\hline $\mathrm{VBEON}(\mathrm{mV}) @ \mathrm{~V}(\mathrm{E}=-10 \mathrm{~V}, \mathrm{IC}=-50 \mathrm{~mA}$ & 874.47 & 870.62 & 3.85 & 1.84 & 1.66 & 0.18 \\
\hline $\mathrm{VF}(\mathrm{mV}) @ \mathrm{IF}=2 \mathrm{~mA}$ & 342.19 & 344.36 & -2.17 & 3.59 & 1.51 & 2.08 \\
\hline $\mathrm{VF}(\mathrm{mV}) @ \mathrm{IF}=10 \mathrm{~mA}$ & 375.55 & 378.38 & .2 .83 & 3.51 & 1.64 & 1.87 \\
\hline $\mathrm{VF}(\mathrm{mV}) @ \mathrm{IF}=100 \mathrm{~mA}$ & 429.52 & 432.78 & -3.26 & 3.42 & 1.53 & 1.89 \\
\hline $\mathrm{VF}(\mathrm{mV}) @ \mathrm{IF}=1 \mathrm{~A}$ & 545.67 & 547.68 & -2.01 & 3.55 & 2.03 & 1.52 \\
\hline $\mathrm{VF}(\mathrm{mV}) @ \mathrm{IF}=2 \mathrm{~A}$ & 638.41 & 638.74 & -0.33 & 4.45 & 3.18 & 1.27 \\
\hline $\mathrm{VF}(\mathrm{mV}) @ \mathrm{IF}=2 \mathrm{~mA}$ & 341.44 & 343.64 & -2.2 & 4.27 & 2.57 & 1.7 \\
\hline $\mathrm{VF}(\mathrm{mV}) @ \mathrm{IF}=10 \mathrm{~mA}$ & 375.01 & 377.59 & -2.58 & 4.23 & 2.84 & 1.39 \\
\hline $\mathrm{VF}(\mathrm{mV}) @ \mathrm{IF}=100 \mathrm{~mA}$ & 428.79 & 432.06 & -3.27 & 4.36 & 2.95 & 1.41 \\
\hline $\mathrm{VF}(\mathrm{mV}) @ \mathrm{IF}=1 \mathrm{~A}$ & 543.16 & 546.57 & -3.41 & 4.66 & 3.79 & 0.87 \\
\hline$V F(m V) @ I F=2 A$ & 633.94 & 637.28 & -3.34 & 5.74 & 5.24 & 0.5 \\
\hline
\end{tabular}




\begin{tabular}{|c|c|c|c|c|c|c|}
\hline TEST & \multicolumn{3}{|c|}{ MINIMUM VALUE } & \multicolumn{3}{|c|}{ MAXIMUM VALUE } \\
\hline & Freon & DI Water & Delta & Freon & DI Water & Delta \\
\hline $\mathrm{ICBO}(\mathrm{nA}) @ \mathrm{VCB}=60 \mathrm{~V}$ & 15 & 16 & -1 & 124 & 74 & 50 \\
\hline IEBO (nA)@VEB $=4 \mathrm{~V}$ & 0.06 & 1.2 & -1.14 & 28 & 18 & 10 \\
\hline $\operatorname{ICER}(\mathrm{nA}) @ \mathrm{VC}=50 \mathrm{~V}, \mathrm{R}=5 \mathrm{~K}$ & 17 & 18 & -1 & 109 & 65 & 44 \\
\hline $\mathrm{HFE} @ \mathrm{VCE}=10 \mathrm{~V}, \mathrm{IC}=20 \mathrm{~mA}$ & 253 & 246 & 7 & 305 & 276 & 29 \\
\hline $\mathrm{HFE} @ \mathrm{VCE}=10 \mathrm{~V}, \mathrm{IC}=50 \mathrm{~mA}$ & 244 & 236 & 8 & 295 & 265 & 30 \\
\hline $\mathrm{VBEON}(\mathrm{mV}) @ \mathrm{VCE}=10 \mathrm{~V}, \mathrm{IC}=20 \mathrm{~mA}$ & 539 & 545 & -6 & 574.5 & 551.5 & 23 \\
\hline VBEON $(\mathrm{mV}) @ \mathrm{VCE}=10 \mathrm{~V}, \mathrm{IC}=50 \mathrm{~mA}$ & 571 & 576.5 & -5.5 & 605 & 583.5 & 21.5 \\
\hline $\mathrm{ICBO}(\mathrm{nA}) @ \mathrm{VCB}=-50 \mathrm{~V}$ & 7 & 4.9 & 2.1 & 23 & 24 & -1 \\
\hline IEBO (nA)@VEB $=-4 V$ & 1.8 & 1.7 & 0.1 & 4.3 & 3 & 1.3 \\
\hline $\operatorname{ICER}(\mathrm{nA}) @ \mathrm{VC}=-50 \mathrm{~V}, \mathrm{R}=5 \mathrm{~K}$ & 9.8 & 6.8 & 3 & 33 & 32 & 1 \\
\hline $\mathrm{HFE} @ \mathrm{VCE}=-10 \mathrm{~V}, \mathrm{IC}=-20 \mathrm{~mA}$ & 187.2 & 207 & -19.8 & 211 & 225 & -14 \\
\hline $\mathrm{HFE} @ \mathrm{VCE}=-10 \mathrm{~V}, \mathrm{IC}=-50 \mathrm{~mA}$ & 186.6 & 206 & -19.4 & 210 & 225 & -15 \\
\hline $\begin{array}{l}\text { VBEON }(\mathrm{mV}) @ \mathrm{VCE}=-10 \mathrm{~V}, \mathrm{IC}=- \\
20 \mathrm{~mA}\end{array}$ & 550.5 & 547 & 3.5 & 580 & 553 & 27 \\
\hline $\begin{array}{l}\text { VBEON }(\mathrm{mV}) @ \mathrm{VCE}=-10 \mathrm{~V}, \mathrm{IC}=- \\
50 \mathrm{~mA}\end{array}$ & 580.5 & 576 & 4.5 & 609.5 & 582 & 27.5 \\
\hline IR $(\mu \mathrm{A}) @ \mathrm{VR}=6 \mathrm{~V}$ & 372 & 687 & -315 & 1090 & 880 & 210 \\
\hline $\mathrm{IR}(\mu \mathrm{A}) @ \mathrm{VR}=36 \mathrm{~V}$ & 872 & 1580 & -708 & 2610 & 2030 & 580 \\
\hline $\mathrm{VF}(\mathrm{mV}) @ \mathrm{IF}=2 \mathrm{~mA}$ & 48.5 & 53.5 & -5 & 76 & 60 & 16 \\
\hline $\mathrm{VF}(\mathrm{mV}) @ \mathrm{IF}=10 \mathrm{~mA}$ & 98 & 103.5 & -5.5 & 127.5 & 111.5 & 16 \\
\hline $\mathrm{VF}(\mathrm{mV}) @ \mathrm{IF}=100 \mathrm{~mA}$ & 190.5 & 196 & -5.5 & 217 & 203.5 & 13.5 \\
\hline $\mathrm{VF}(\mathrm{mV}) @ I \mathrm{I}=450 \mathrm{~mA}$ & 298.5 & 302.5 & -4 & 321 & 311 & 10 \\
\hline $\mathrm{IR}(\mu \mathrm{A}) @ \mathrm{VR}=6 \mathrm{~V}$ & 365 & 665 & -300 & 1300 & 1040 & 260 \\
\hline $\mathrm{IR}(\mu \mathrm{A}) @ \mathrm{VR}=36 \mathrm{~V}$ & 868 & 1520 & -652 & 3090 & 2360 & 730 \\
\hline $\mathrm{VF}(\mathrm{mV}) @ \mathrm{IF}=2 \mathrm{~mA}$ & 44 & 49.5 & -5.5 & 76 & 60.5 & 15.5 \\
\hline$V F(m V) @ I F=10 \mathrm{~mA}$ & 92 & 98 & .6 & 127.5 & 112 & 15.5 \\
\hline $\mathrm{VF}(\mathrm{mV}) @ \mathrm{IF}=100 \mathrm{~mA}$ & 183 & 189.5 & -6.5 & 216.5 & 204.5 & 12 \\
\hline $\mathrm{VF}(\mathrm{mV}) @ \mathrm{IF}=450 \mathrm{mV}$ & 289 & 295 & -6 & 320.5 & 312.5 & 8 \\
\hline
\end{tabular}


TABLE A12: AVERAGE AND STANDARD DEVIATION OF ELECTRICAL TEST: DATA AT $100^{\circ} \mathrm{C}$ FOR M-53

\begin{tabular}{|c|c|c|c|c|c|c|}
\hline \multirow[t]{2}{*}{ TEST } & \multicolumn{3}{|c|}{ AVERAGE VALUE } & \multicolumn{3}{|c|}{ STANDARD DEVIATION } \\
\hline & Freon & DI Water & Delta & Freon & DI Water & Delta \\
\hline $\mathrm{ICBO}(\mathrm{nA}) @ \mathrm{VCB}=60 \mathrm{~V}$ & 31 & 41 & -10 & 13 & 13 & 0 \\
\hline IEBO $(\mathrm{nA}) @ \mathrm{VEB}=4 \mathrm{~V}$ & 3.3 & 2.8 & 0.5 & 4 & 2.3 & 1.7 \\
\hline $\operatorname{ICER}(\mathrm{nA}) @ \mathrm{VC}=50 \mathrm{~V}, \mathrm{R}=5 \mathrm{~K}$ & 34 & 41 & -7 & 12 & 12 & 0 \\
\hline HFE@VCE $=10 \mathrm{~V}, \mathrm{IC}=20 \mathrm{~mA}$ & 286.61 & 259.71 & 26.9 & 15.72 & 7.5 & 8.12 \\
\hline $\mathrm{HFE} @ \mathrm{VCE}=10 \mathrm{~V}, \mathrm{IC}=50 \mathrm{~mA}$ & 276.69 & 249.25 & 27.44 & 15.83 & 7.49 & 8.34 \\
\hline VBEON(mV)@VCE $=10 \mathrm{~V}, \mathrm{IC}=20 \mathrm{~mA}$ & 547.44 & 549.19 & -1.75 & 6.74 & 1.48 & 5.26 \\
\hline VBEON $(\mathrm{mV}) @ V C E=10 \mathrm{~V}, \mathrm{IC}=50 \mathrm{~mA}$ & 579.44 & 580.74 & -1.3 & 6.39 & 1.48 & 4.91 \\
\hline $\mathrm{ICBO}(\mathrm{nA}) @ \mathrm{VCB}=-50 \mathrm{~V}$ & 14 & 7.1 & 6.9 & 3.3 & 2.8 & 0.5 \\
\hline IEBO (nA)@VEB $=-4 \mathrm{~V}$ & 3 & 2.3 & 0.7 & 0.54 & 0.27 & 0.27 \\
\hline $\operatorname{ICER}(\mathrm{nA}) @ V C=-50 \mathrm{~V}, \mathrm{R}=5 \mathrm{~K}$ & 20 & 9.9 & 10.1 & 5.1 & 3.4 & 1.7 \\
\hline $\mathrm{HFE} @ \mathrm{VCE}=-10 \mathrm{~V}, \mathrm{IC}=-20 \mathrm{~mA}$ & 200.31 & 21839 & -18.08 & 4.55 & 3.84 & 0.71 \\
\hline $\mathrm{HFE} @ \mathrm{VCE}=-10 \mathrm{~V}, \mathrm{IC}=-50 \mathrm{~mA}$ & 199.68 & 217.61 & -17.93 & 4.59 & 3.83 & .76 \\
\hline $\begin{array}{l}\mathrm{VBEON}(\mathrm{mV}) @ \mathrm{VCE}=-10 \mathrm{~V}, \mathrm{IC}=- \\
20 \mathrm{~mA}\end{array}$ & 556.69 & 550.78 & 5.91 & 6.26 & 1.33 & 4.93 \\
\hline $\begin{array}{l}\text { VBEON }(\mathrm{mV}) @ \mathrm{VCE}=-10 \mathrm{~V}, \mathrm{IC}=- \\
50 \mathrm{~mA}\end{array}$ & 586.62 & 579.86 & 6.76 & 6.11 & 1.26 & 4.85 \\
\hline IR $(\mu \mathrm{A}) @ \mathrm{VR}=6 \mathrm{~V}$ & 804 & 747 & 57 & 145 & 41.6 & 103.4 \\
\hline $\mathrm{IR}(\mu \mathrm{A}) @ \mathrm{VR}=36 \mathrm{~V}$ & 1840 & 1720 & 120 & 323 & 95.2 & 227.8 \\
\hline $\mathrm{VF}(\mathrm{mV}) @ \mathrm{IF}=2 \mathrm{~mA}$ & 56.57 & 57.69 & -1.12 & 5.42 & 1.58 & 3.84 \\
\hline $\mathrm{VF}(\mathrm{mV}) @ \mathrm{IF}=10 \mathrm{~mA}$ & 106.99 & 108.58 & -1.59 & 5.94 & 1.71 & 4.23 \\
\hline $\mathrm{VF}(\mathrm{mV}) @ \mathrm{IF}=100 \mathrm{~mA}$ & 199.06 & 200.55 & -1.49 & 5.3 & 1.68 & 3.62 \\
\hline $\mathrm{VF}(\mathrm{mV}) @ \mathrm{IF}=450 \mathrm{~mA}$ & 306.9 & 307.59 & -0.69 & 4.51 & 2.08 & 2.43 \\
\hline $\mathrm{IR}(\mu \mathrm{A}) @ \mathrm{VR}=6 \mathrm{~V}$ & 812 & 759 & 53 & 154 & 65.2 & 88.8 \\
\hline $\mathrm{IR}(\mu \mathrm{A}) @ \mathrm{VR}=36 \mathrm{~V}$ & $187 \mathrm{U}$ & 1750 & 120 & 340 & 145 & 195 \\
\hline $\mathrm{VF}(\mathrm{mV}) @ \mathrm{IF}=2 \mathrm{~mA}$ & 56.18 & 57.37 & -1.19 & 5.64 & 2.2 & 3.44 \\
\hline $\mathrm{VF}(\mathrm{mV}) @ I F=10 \mathrm{~mA}$ & 106.47 & -108.13 & -1.66 & 6.12 & 2.53 & 3.59 \\
\hline $\mathrm{VP}(\mathrm{mV}) @ \mathrm{IF}=100 \mathrm{~mA}$ & 198.18 & 200.06 & -1.88 & 5.62 & 2.68 & 2.94 \\
\hline $\mathrm{VF}(\mathrm{mV}) @ \mathrm{IF}=45 \mathrm{mV}$ & 304.61 & 306.82 & -2.21 & 5.14 & 3.25 & $\therefore 1.89$ \\
\hline
\end{tabular}




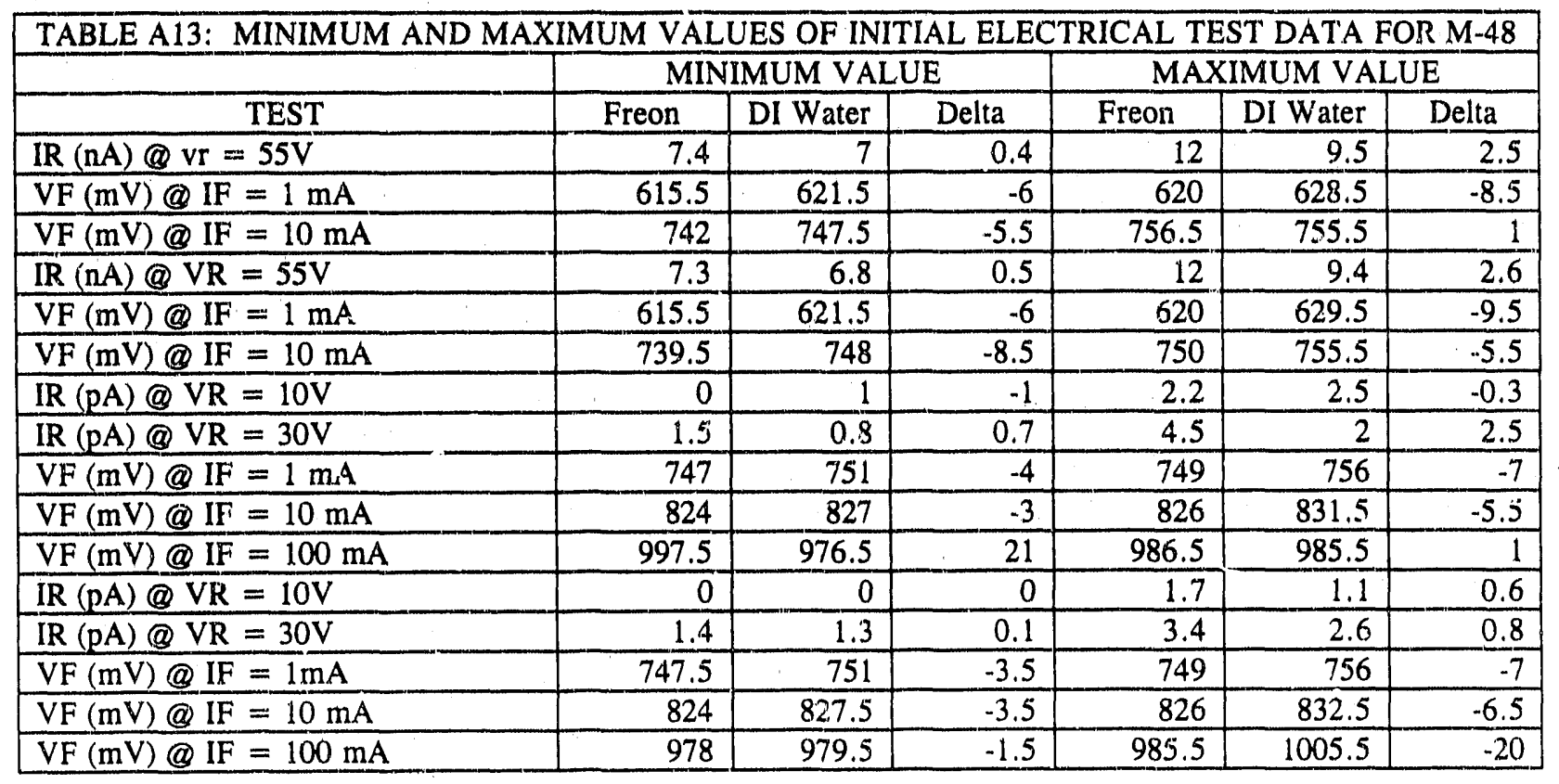

\begin{tabular}{|c|c|c|c|c|c|c|}
\hline & \multicolumn{3}{|c|}{ AVERAGE VALUE } & \multicolumn{3}{|c|}{ STANDARD DEVIATION } \\
\hline TEST & Freon & DI Water & Delta & Freon & DI Water & Delta \\
\hline $\mathrm{IR}(\mathrm{nA}) @ \mathrm{vr}=55 \mathrm{~V}$ & 10 & 8.3 & 1.7 & 0.93 & 0.76 & 0.17 \\
\hline $\mathrm{VF}(\mathrm{mV}) @ \mathrm{IF}=1 \mathrm{~mA}$ & 618.08 & 624.31 & -6.23 & 1.01 & 2.2 & -1.19 \\
\hline $\mathrm{VF}(\mathrm{mV}) @ \mathrm{IF}=10 \mathrm{~mA}$ & 746.45 & 751.22 & 4.77 & 2.1 & 1.88 & 0.22 \\
\hline $\mathrm{IR}(\mathrm{nA}) @ \mathrm{VR}=55 \mathrm{~V}$ & 9.7 & 8.2 & 1.5 & 1.1 & 0.73 & 0.37 \\
\hline $\mathrm{VF}(\mathrm{mV}) @ \mathrm{IF}=1 \mathrm{~mA}$ & 618.2 & 624.54 & -6.34 & $\overline{1.14}$ & 2.29 & -1.15 \\
\hline $\mathrm{VF}(\mathrm{mV}) @ \mathrm{IF}=10 \mathrm{~mA}$ & 745.76 & 751.15 & -5.39 & 2.27 & 1.91 & 0.36 \\
\hline $\mathrm{IR}(\mathrm{pA}) @ \mathrm{VR}=10 \mathrm{~V}$ & 0.53 & 1.5 & -0.97 & 0.44 & 0.24 & 0.2 \\
\hline $\mathrm{IR}(\mathrm{pA}) @ \mathrm{VR}=30 \mathrm{~V}$ & 2.6 & 1.5 & 1.1 & 0.49 & 0.25 & 0.24 \\
\hline $\mathrm{VF}(\mathrm{mV}) @ \mathrm{IF}=1 \mathrm{~mA}$ & 748.12 & 753.04 & -4.92 & 0.33 & 1.68 & -1.35 \\
\hline $\mathrm{VF}(\mathrm{mV}) @ \mathrm{IF}=10 \mathrm{~mA}$ & 824.63 & 828.72 & -4.09 & 0.53 & 1.44 & -0.91 \\
\hline $\mathrm{VF}(\mathrm{mV}) @ \mathrm{IF}=100 \mathrm{~mA}$ & 980.52 & 980.48 & 0.04 & 1.78 & 2.23 & -0.45 \\
\hline $\mathrm{IR}(\mathrm{pA}) @ \mathrm{VR}=10 \mathrm{~V}$ & 0.55 & 0.16 & 0.39 & 0.35 & 0.16 & 0.19 \\
\hline $\mathrm{IR}(\mathrm{pA}) \mathrm{VR}=30 \mathrm{~V}$ & 2 & 2 & 0 & 0.35 & 0.28 & 0.07 \\
\hline $\mathrm{VF}(\mathrm{mV}) @ \mathrm{IF}=1 \mathrm{~mA}$ & 748.15 & 752.88 & -4.73 & 0.26 & 1.62 & -1.36 \\
\hline $\mathrm{VF}(\mathrm{mV}) @ \mathrm{IF}=10 \mathrm{~mA}$ & 825.21 & 829.35 & -4.14 & 0.50 & 1.47 & -0.97 \\
\hline $\mathrm{VF}(\mathrm{mV}) @ \mathrm{IF}=100 \mathrm{~mA}$ & 982.3 & 984.35 & .2 .05 & 1.78 & 3.66 & -1.88 \\
\hline
\end{tabular}




\begin{tabular}{|c|c|c|c|c|c|c|}
\hline & \multicolumn{3}{|c|}{ MINIMUM VALUE } & \multicolumn{3}{|c|}{ MAXIMUM 'ALUE } \\
\hline TEST & Freon & DI Water & Delta & Freon & DI Water & Delta \\
\hline $\mathrm{IR}(\mathrm{nA}) @ \mathrm{vr}=55 \mathrm{~V}$ & 8.9 & $7 . \overline{2}$ & 1.7 & 15 & 9.9 & 5.1 \\
\hline $\mathrm{VF}(\mathrm{mV}) @ \mathrm{IF}=1 \mathrm{~mA}$ & 617.5 & 624 & -6.5 & 624 & 629.5 & -5.5 \\
\hline $\mathrm{VF}(\mathrm{mV}) @ \mathrm{IF}=10 \mathrm{~mA}$ & 742 & 750 & -8 & 751.5 & 755.5 & -4 \\
\hline $\mathrm{IR}(\mathrm{nA}) @ \mathrm{VR}=55 \mathrm{~V}$ & 8 & 7.1 & 0.9 & 16 & 9.8 & 6.2 \\
\hline $\mathrm{VF}(\mathrm{mV}) @ \mathrm{IF}=1 \mathrm{~mA}$ & 616.5 & 625 & -8.5 & 622 & 629.5 & -7.5 \\
\hline $\mathrm{VF}(\mathrm{mV}) @ \mathrm{IF}=10 \mathrm{~mA}$ & 741.5 & 751.5 & -10 & 751.5 & 755.5 & -4 \\
\hline $\mathrm{IR}(\mathrm{pA}) @ \mathrm{VR}=10 \mathrm{~V}$ & 0 & 1.1 & -1.1 & 0.6 & 1.8 & -1.2 \\
\hline $\mathrm{IR}(\mathrm{pA}) @ \mathrm{VR}=30 \mathrm{~V}$ & 1.7 & 0.8 & 0.9 & 3.5 & 1.8 & 1.7 \\
\hline $\mathrm{VF}(\mathrm{mV}) @ \mathrm{IF}=1 \mathrm{~mA}$ & 748 & 753.5 & -5.5 & 752 & 756.5 & -4.5 \\
\hline $\mathrm{VF}(\mathrm{mV}) @ \mathrm{IF}=10 \mathrm{~mA}$ & 842.5 & 829 & 13.5 & 828 & 831.5 & -3.5 \\
\hline$\overline{V F}(\mathrm{mV}) @ \mathrm{IF}=100 \mathrm{~mA}$ & 977.5 & 978.5 & -1 & 986.5 & 986 & 0.5 \\
\hline $\mathrm{IR}(\mathrm{pA}) @ \mathrm{VR}=10 \mathrm{~V}$ & 0 & 0 & 0 & 0.6 & 0.5 & 0.1 \\
\hline $\mathrm{IR}(\mathrm{pA}) @ \mathrm{VR}=30 \mathrm{~V}$ & 1.5 & 1.1 & 0.4 & 2.4 & 2.1 & 0.3 \\
\hline $\mathrm{VF}(\mathrm{mV}) @ \mathrm{IF}=1 \mathrm{~mA}$ & 748 & 753.5 & -5.5 & 752 & 756 & -4 \\
\hline $\mathrm{VF}(\mathrm{mV}) @ \mathrm{IF}=10 \mathrm{~mA}$ & 824.5 & 830 & -5.5 & 828.5 & 832 & -3.5 \\
\hline$V F(m V) @ I F=100 \mathrm{~mA}$ & 978 & 981 & -3 & 986.5 & 988.5 & -2 \\
\hline
\end{tabular}

\begin{tabular}{|c|c|c|c|c|c|c|}
\hline & \multicolumn{3}{|c|}{ AVERAGE VALUE } & \multicolumn{3}{|c|}{ STANDARD DEVIATION } \\
\hline TEST & Freon & DI Water & Delta & Freon & DI Water & Delta \\
\hline $\mathrm{IR}(\mathrm{nA}) @ \mathrm{vr}=55 \mathrm{~V}$ & 12 & 9.3 & 2.7 & 1.4 & 0.61 & 0.79 \\
\hline$V F(\mathrm{mV}) @ I F=1 \mathrm{~mA}$ & 619.48 & 627.16 & -7.68 & 1.25 & 0.98 & 0.27 \\
\hline $\mathrm{VF}(\mathrm{mV}) @ \mathrm{IF}=10 \mathrm{~mA}$ & 747.43 & 753.29 & -5.86 & 1.85 & 1.11 & 0.74 \\
\hline $\mathrm{IR}(\mathrm{nA}) @ \mathrm{VR}=55 \mathrm{~V}$ & 11 & 8.2 & 2.8 & 1.7 & 0.58 & 1.12 \\
\hline $\mathrm{VF}(\mathrm{mV}) @ \mathrm{IF}=1 \mathrm{~mA}$ & 619.69 & 627.26 & -7.57 & 1.14 & 0.95 & 0.19 \\
\hline $\mathrm{VF}(\mathrm{mV}) @ \mathrm{IF}=10 \mathrm{~mA}$ & 746.86 & 753.29 & -6.43 & 2.24 & 0.98 & 1.26 \\
\hline $\mathrm{IR}(\mathrm{p} A) @ \mathrm{VR}=10 \mathrm{~V}$ & 0.3 & 1.4 & -1.1 & 0.18 & 0.15 & 0.03 \\
\hline$I R(p A) @ V R=30 V$ & $\overline{2.3}$ & 1.4 & 0.9 & 0.29 & 0.19 & 0.1 \\
\hline $\mathrm{VF}(\mathrm{mV}) @ \mathrm{IF}=1 \mathrm{~mA}$ & 749.39 & 755.11 & -5.72 & 0.93 & 0.69 & 0.24 \\
\hline $\mathrm{VF}(\mathrm{mV}) @ \mathrm{IF}=10 \mathrm{~mA}$ & 825.67 & 830.53 & -486 & 0.69 & 0.66 & 0.03 \\
\hline$V F(\mathrm{mV}) @ \mathrm{IF}=100 \mathrm{~mA}$ & 980.98 & 981.82 & -0.84 & 1.8 & 1.85 & -0.05 \\
\hline $\mathrm{IR}(\mathrm{pA}) @ \mathrm{VR}=10 \mathrm{~V}$ & 0.14 & 0.15 & -0.01 & 0.12 & 0.11 & 0.01 \\
\hline$I R(p A) @ V R=30 V$ & 1.9 & 1.6 & 0.3 & 0.24 & 0.19 & 0.05 \\
\hline $\mathrm{VF}(\mathrm{mV}) @ \mathrm{IF}=1 \mathrm{~mA}$ & 749.47 & 755.01 & -5.54 & 0.91 & 0.68 & 0.23 \\
\hline $\mathrm{VF}(\mathrm{mV}) @ \mathrm{IF}=10 \mathrm{~mA}$ & 826.23 & 831.21 & 4.98 & 0.88 & 0.59 & 0.59 \\
\hline $\mathrm{VF}(\mathrm{mV}) @ \mathrm{IF}=100 \mathrm{~mA}$ & $98 z .88$ & 985.03 & -2.15 & 1.84 & 1.54 & 0.3 \\
\hline
\end{tabular}




\begin{tabular}{|l|r|r|r|r|r|}
\hline \multicolumn{1}{|c|}{ TABLE A17: MINIMUM AND MAXIMUM VALUES OF POST EXTENDED HTRB ELECTRICAL TEST } \\
\hline \\
\hline
\end{tabular}

\begin{tabular}{|c|c|c|c|c|c|c|}
\hline & \multicolumn{3}{|c|}{ AVERAGE VALUE } & \multicolumn{3}{|c|}{ STANDAED DEVIATION } \\
\hline TEST & Freon & D! Water & Delta & Freon & DI Water & Delta \\
\hline $\mathrm{IR}(\mathrm{nA}) @ \mathrm{vr}_{\mathrm{r}}=55 \mathrm{~V}$ & 12 & 9.3 & 2.7 & 1.3 & 0.76 & 0.54 \\
\hline $\mathrm{VF}(\mathrm{mV}) @ \mathrm{IF}=1 \mathrm{~mA}$ & 621.48 & 625.15 & -3.67 & 1.62 & 1.36 & 0.26 \\
\hline $\mathrm{VF}(\mathrm{mV}) @ \mathrm{IF}=10 \mathrm{~mA}$ & 749.28 & 751.85 & -2.57 & 1.94 & $1 . \overline{45}$ & 0.49 \\
\hline$\overline{L R}(\mathrm{nA}) @ \mathrm{VR}=55 \mathrm{~V}$ & 11 & 9.3 & 1.7 & 1.8 & 0.8 & 1 \\
\hline $\mathrm{VF}(\mathrm{mV}) @ \mathrm{IF}=1 \mathrm{~mA}$ & 621.25 & 625.48 & -4.23 & $1.4^{\prime} 7$ & 0.94 & 0.53 \\
\hline $\mathrm{VF}(\mathrm{mV}) @ \mathrm{IF}=10 \mathrm{~mA}$ & 748.03 & 752.1 & -4.07 & 2.68 & 0.36 & 2.32 \\
\hline $\mathrm{IR}(\mathrm{pA}) @ \mathrm{VR}=10 \mathrm{~V}$ & 1.9 & 1.9 & 9 & 0.69 & 0.36 & 0.33 \\
\hline $\mathrm{IR}(\mathrm{pA}) @ \mathrm{VR}=30 \mathrm{~V}$ & 2.1 & .76 & 1.34 & 1 & 0.23 & 0.77 \\
\hline $\mathrm{VF}(\mathrm{mV}) @ I \mathrm{I}=1 \mathrm{~mA}$ & 750.68 & 753.48 & -2.8 & 1.23 & 0.84 & 0.39 \\
\hline $\mathrm{VF}(\mathrm{mV}) @ \mathrm{IF}=10 \mathrm{~mA}$ & 826.85 & 829.1 & -2.25 & 1.09 & 0.85 & $0 . \overline{24}$ \\
\hline $\mathrm{VF}(\mathrm{mV}) @ \mathrm{IF}=100 \mathrm{~mA}$ & 981.48 & 981.69 & -0.21 & 1.63 & 2.12 & -0.49 \\
\hline $\mathrm{IR}(\mathrm{pA}) @ \mathrm{VR}=10 \mathrm{~V}$ & 0.58 & 0.87 & -0.29 & 0.75 & 0.23 & 0.52 \\
\hline $\mathrm{IR}(\mathrm{pA}) @ \mathrm{VR}=30 \mathrm{~V}$ & 1.9 & 0.94 & $0 . \overline{96}$ & 0.41 & 0.26 & 0.15 \\
\hline $\mathrm{VF}(\mathrm{mV}) @ \mathrm{IF}=1 \mathrm{~mA}$ & 750.98 & 753.21 & -2.23 & 1.04 & 0.66 & 0.38 \\
\hline $\mathrm{VF}(\mathrm{mV}) @ \mathrm{IF}=\mathrm{l} \mathrm{mA}$ & 827.65 & 830.06 & -2.41 & 0.99 & 0.67 & 0.32 \\
\hline $\mathrm{VF}(\mathrm{mV}) @ \mathrm{IF}=100 \mathrm{~mA}$ & 984.35 & 984.35 & 0 & 1.62 & 1.65 & -0.03 \\
\hline
\end{tabular}




\begin{tabular}{|c|c|c|c|c|c|c|}
\hline & \multicolumn{3}{|c|}{ MINIMUM VALUE } & \multicolumn{3}{|c|}{ MAXIMUM VALUE } \\
\hline TEST & Freon & DI Water & Delta & Freon & DI Water & Delta \\
\hline $\mathrm{IR}(\mathrm{n} \overline{\mathrm{A}}) @ \mathrm{vr}=55 \mathrm{~V}$ & 8.7 & 7.4 & 1.3 & 12 & 9.7 & 2.3 \\
\hline $\mathrm{VF}(\mathrm{mV}) @ \mathrm{IF}=1 \mathrm{~mA}$ & 621 & 627 & -6 & 625 & 630 & -5 \\
\hline $\mathrm{VF}(\mathrm{mV}) @ \mathrm{IF}=10 \mathrm{~mA}$ & 748 & 752 & -4 & 753.5 & 756 & -2.5 \\
\hline $\mathrm{IR}(\mathrm{nA}) @ \mathrm{VR}=55 \mathrm{~V}$ & 7.4 & 7.4 & 0 & 13 & 9.3 & 3.7 \\
\hline $\mathrm{VF}(\mathrm{mV}) @ \mathrm{IF}=1 \mathrm{~mA}$ & 622 & 628.5 & -6.5 & 625 & 630 & -5 \\
\hline $\mathrm{VF}\left(\mathrm{m}^{\mathrm{v}} \mathrm{)} @ \mathrm{IF}=10 \mathrm{~mA}\right.$ & 744.5 & 754 & -9.5 & 7,54 & 755.5 & -1.5 \\
\hline $\mathrm{IR}(\mathrm{pA}) @ \mathrm{VR}=10 \mathrm{~V}$ & 1.4 & 0.8 & 0.6 & 2.3 & 2.5 & -0.2 \\
\hline$I R(p A) @ V R=30 V$ & 1.5 & 0.2 & 1.3 & 2.1 & 1.9 & 0.2 \\
\hline $\mathrm{VF}(\mathrm{mV}) @ \mathrm{IF}=1 \mathrm{~mA}$ & 752 & 756 & -4 & 752.5 & 756.5 & -4 \\
\hline $\mathrm{VF}(\mathrm{mV}) @ \mathrm{IF}=10 \mathrm{~mA}$ & 828 & 831.5 & -3.5 & 828.5 & 832 & -3.5 \\
\hline $\mathrm{VF}(\mathrm{mV}) @ \mathrm{IF}=100 \mathrm{~mA}$ & 980.5 & 980.5 & 0 & 986 & 986.5 & -0.5 \\
\hline $\mathrm{IR}(\mathrm{pA}) @ \mathrm{VR}=10 \mathrm{~V}$ & 0 & 0.2 & -0.2 & 0.4 & 1.4 & -1 \\
\hline $\mathrm{IR}(\mathrm{pA}) @ \mathrm{VR}=30 \mathrm{~V}$ & 1.7 & 0 & 1.7 & 2.8 & 1.5 & 1.3 \\
\hline $\mathrm{VF}(\mathrm{mV}) @ \mathrm{IF}=1 \mathrm{~mA}$ & 752 & 756 & .4 & 753 & 757 & -4 \\
\hline $\mathrm{VF}(\mathrm{mV}) @ \mathrm{IF}=10 \mathrm{~mA}$ & 828 & 831.5 & -3.5 & 830 & 833 & -3 \\
\hline$\overline{V F}(\mathrm{mV}) @ \mathrm{IF}=100 \mathrm{~mA}$ & 981 & 983.5 & -2.5 & 987.5 & 989.5 & -2 \\
\hline
\end{tabular}

\begin{tabular}{|c|c|c|c|c|c|c|}
\hline & \multicolumn{3}{|c|}{ AVERAGE VALUE } & \multicolumn{3}{|c|}{ STANDARD DEVIATION } \\
\hline $\begin{array}{r}\text { TEST } \\
\end{array}$ & Freon & DI Water & Delta & Freon & DI Water & Delta \\
\hline $\mathrm{IR}(\mathrm{nA}) @ \mathrm{vr}=55 \mathrm{~V}$ & 10 & 8.6 & 1.4 & 1.1 & 0.75 & 0.35 \\
\hline $\mathrm{VF}(\mathrm{mV}) @ \mathrm{IF}=1 \mathrm{~mA}$ & 623.73 & 629.13 & -5.4 & 0.97 & 0.86 & 0.11 \\
\hline $\mathrm{VF}(\mathrm{mV}) @ \mathrm{IF}=10 \mathrm{~mA}$ & 750.65 & 754.93 & -4.28 & 1.42 & 1.05 & 0.37 \\
\hline $\mathrm{IR}(\mathrm{nA}) @ \mathrm{VR}=55 \mathrm{~V}$ & 10 & 8.5 & 1.5 & 1.5 & 0.6 & 0.9 \\
\hline $\mathrm{VF}(\mathrm{mV}) @ \mathrm{IF}=1 \mathrm{~mA}$ & 623.85 & 629.65 & -5.8 & 0.78 & 0.49 & 29 \\
\hline $\mathrm{VF}(\mathrm{mV}) @ \mathrm{IF}=10 \mathrm{~mA}$ & 750.43 & 755.18 & 4.75 & 2.46 & 0.55 & 1.91 \\
\hline$I R(p A) @ V R=10 V$ & 1.8 & 1.5 & 0.3 & 0.21 & 0.36 & -0.15 \\
\hline $\mathrm{IR}(\mathrm{pA}) @ \mathrm{VR}=30 \mathrm{~V}$ & 1.7 & 0.82 & 0.88 & 0.21 & 0.49 & -0.28 \\
\hline $\mathrm{VF}(\mathrm{mV}) @ \mathrm{IF}=1 \mathrm{~mA}$ & 752.1 & 756.48 & -4.38 & 0.21 & 0.11 & 0.10 \\
\hline $\bar{V} \bar{F}(\mathrm{mV}) @ \mathrm{IF}=10 \mathrm{~mA}$ & 828.15 & 831.6 & -3.45 & 0.24 & 0.21 & 0.03 \\
\hline $\mathrm{VF}(\mathrm{mV}) @ \mathrm{IF}=100 \mathrm{~mA}$ & 982.9 & 983.03 & -0.13 & 1.49 & 1.93 & -0.44 \\
\hline $\mathrm{IR}(\mathrm{pA}) @ \mathrm{VR}=10 \mathrm{~V}$ & 0.12 & 0.58 & -0.46 & 0.11 & 0.28 & -0.17 \\
\hline $\mathrm{IR}(\mathrm{pA}) @ \mathrm{VR}=30 \mathrm{~V}$ & 2.2 & 0.9 & 1.3 & 0.3 & 0.39 & -0.09 \\
\hline $\mathrm{VF}(\mathrm{mV}) @ \mathrm{IF}=1 \mathrm{~mA}$ & 752.15 & 756.45 & -4.3 & 0.29 & 0.22 & 0.07 \\
\hline $\mathrm{VF}(\mathrm{mV}) @ \mathrm{IF}=10 \mathrm{~mA}$ & 828.75 & 832.25 & -3.5 & 0.6 & 0.38 & 0.22 \\
\hline $\mathrm{VF}(\mathrm{mV}) @ \mathrm{IF}=100 \mathrm{~mA}$ & 984.93 & 985.75 & -0.82 & 1.64 & 1.56 & 0.08 \\
\hline
\end{tabular}




\begin{tabular}{|c|c|c|c|c|c|c|}
\hline & \multicolumn{3}{|c|}{ MINIMUM VALUE } & \multicolumn{3}{|c|}{ MAXIMUM VALUE } \\
\hline TEST & Freon & DI Water & Delta & Freon & DI Water & Delta \\
\hline $\mathrm{VF}(\mathrm{mV}) @ \mathrm{IF}=1 \mathrm{~mA}$ & 778 & 771.5 & 6.5 & 785 & 786 & -1 \\
\hline $\mathrm{VF}(\mathrm{mV}) @ \mathrm{IF}=10 \mathrm{~mA}$ & 872 & 867 & 5 & 879 & 879 & 0 \\
\hline $\mathrm{VF}(\mathrm{mV}) @ \mathrm{IF}=25 \mathrm{~mA}$ & 930 & 925 & 5 & 938 & 935.5 & 2.5 \\
\hline $\mathrm{VF}(\mathrm{mV}) @ \mathrm{IF}=1 \mathrm{~mA}$ & 778 & 771.5 & 6.5 & 785.5 & 786 & -0.5 \\
\hline $\mathrm{VF}(\mathrm{mlV}) @ \mathrm{IF}=10 \mathrm{~mA}$ & 870 & 867 & 3 & 879 & 879 & 0 \\
\hline $\mathrm{VF}(\mathrm{mV}) @ \mathrm{IF}=25 \mathrm{~mA}$ & 926 & 925 & $\overline{1}$ & 938 & 936 & 2 \\
\hline$\overline{V F}(\mathrm{mV}) @ \mathrm{IF}=1 \mathrm{~mA}$ & 876 & 870 & 6 & 881.5 & 881.5 & 0 \\
\hline $\mathrm{VF}(\mathrm{mV}) @ \mathrm{IF}=10 \mathrm{~mA}$ & 935 & 928.5 & 6.5 & 940 & 940 & 0 \\
\hline $\mathrm{VF}(\mathrm{mV}) @ \mathrm{IF}=1 \mathrm{~mA}$ & 876 & 870 & 6 & 882 & 881.5 & 0.5 \\
\hline $\mathrm{VF}(\mathrm{mV}) @ \mathrm{IF}=10 \mathrm{~mA}$ & 935.5 & 929.5 & 6 & 977.5 & 939.5 & 38 \\
\hline
\end{tabular}

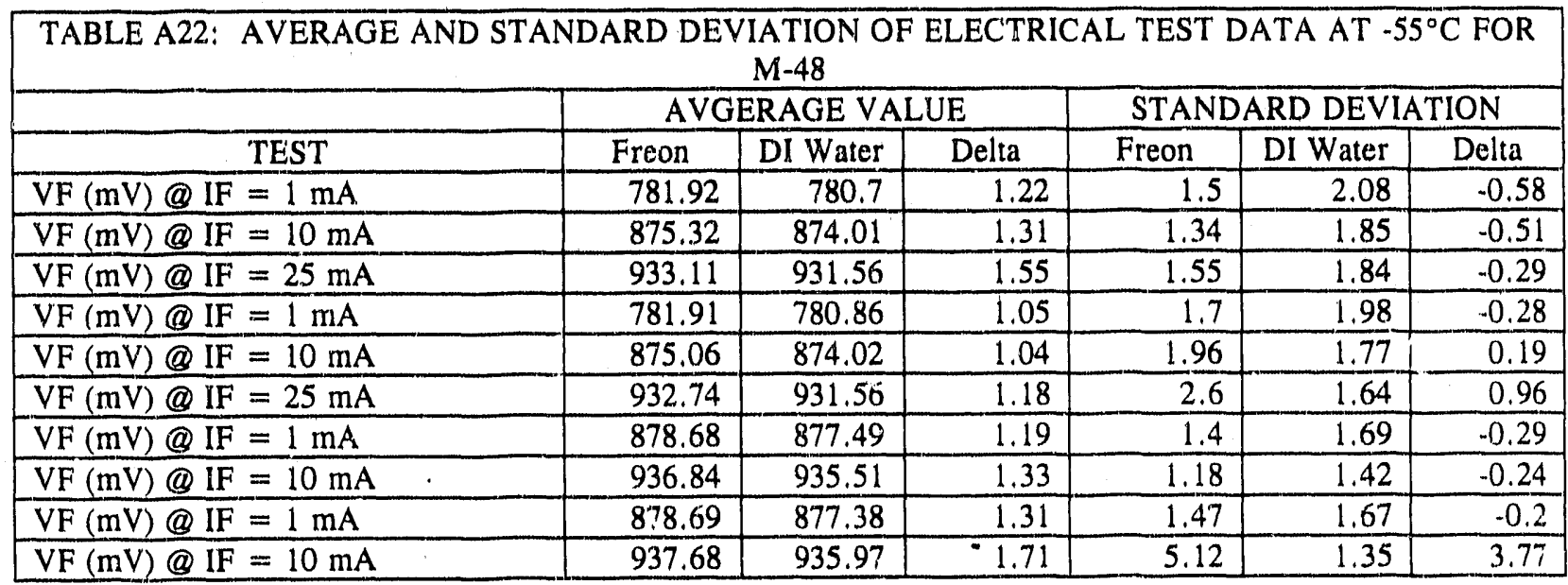




\begin{tabular}{|c|c|c|c|c|c|c|}
\hline & \multicolumn{3}{|c|}{ MINIMUM VALUE } & \multicolumn{3}{|c|}{ MAXIMUM VALUE } \\
\hline TEST & Freon & DI Water & Delta & Freon & DI Water & Delta \\
\hline $\mathrm{IR}(\mathrm{nA}) @ \mathrm{vr}=55 \mathrm{~V}$ & 5.05 & 5.53 & .0 .48 & 17,6 & 7.91 & 9.69 \\
\hline $\mathrm{VF}(\mathrm{mV}) @ \mathrm{IF}=1 \mathrm{~mA}$ & 404.5 & 404.5 & 0 & 415.5 & 415.5 & 0 \\
\hline $\mathrm{VF}(\mathrm{mV}) @ \mathrm{IF}=10 \mathrm{~mA}$ & 572 & 572.5 & -0.5 & 582 & 582 & 0 \\
\hline $\mathrm{IR}(\mathrm{nA}) @ \mathrm{VR}=55 \mathrm{~V}$ & 4,44 & 5.57 & $-1,13$ & 8.76 & 8.26 & 0.5 \\
\hline $\mathrm{VF}(\mathrm{mV}) @ I F=1 \mathrm{~mA}$ & 407 & 404.5 & 2.5 & 417.5 & 415.5 & 2 \\
\hline$\overline{V F}(\mathrm{mV}) @ \mathrm{IF}=10 \mathrm{~mA}$ & 575.5 & 574 & 1.5 & 580.5 & 582 & -1.5 \\
\hline $\mathrm{IR}(\mathrm{pA}) @ \mathrm{VR}=10 \mathrm{~V}$ & 0.17 & 0.15 & 0.02 & 1.3 & 0.51 & 0.79 \\
\hline $\mathrm{IR}(\mathrm{pA}) @ \mathrm{VR}=30 \mathrm{~V}$ & 0.78 & 0.32 & 0.46 & 4.3 & 1.7 & 2.6 \\
\hline $\mathrm{VF}(\mathrm{mV}) @ \mathrm{IF}=1 \mathrm{~mA}$ & 574 & 572 & 2 & 579.5 & 582 & -2.5 \\
\hline $\mathrm{VF}(\mathrm{mV}) @ \mathrm{IF}=10 \mathrm{~mA}$ & 674 & 672.5 & $1 . \overline{5}$ & 679.5 & 700 & -20.5 \\
\hline $\mathrm{IR}(\mathrm{pA}) @ \mathrm{VR}=10 \mathrm{~V}$ & 0.2 & 0.13 & 0.07 & 3 & 1.7 & 1.3 \\
\hline $\mathrm{IR}(\mathrm{pA}) @ \mathrm{VR}=30 \mathrm{~V}$ & 1.2 & 0.32 & 0.88 & 9,8 & 5.3 & 4.5 \\
\hline $\mathrm{VF}(\mathrm{mV}) @ \mathrm{IF}=1 \mathrm{~mA}$ & 574 & 572 & 2 & 580 & 582 & -2 \\
\hline$V F(\mathrm{mV}) @ I F=10 \mathrm{~mA}$ & 675.5 & 673 & 2.5 & 680.5 & 682 & -1.5 \\
\hline
\end{tabular}

\begin{tabular}{|c|c|c|c|c|c|c|}
\hline \multicolumn{7}{|c|}{$\begin{array}{l}\text { TABLE A24: AVERAGE AND STANDARD DEVIATION OF ELECTRICAL TEST DATA AT } 100^{\circ} \mathrm{C} \\
\text { FOR M-48 }\end{array}$} \\
\hline & \multicolumn{3}{|c|}{ AVERAGE VALUE } & \multicolumn{3}{|c|}{ STANDARD DEVIATION } \\
\hline TEST & Freon & DI Water & Delta & Freon & DI Water & Delta \\
\hline IR $(13 \mathrm{~A}) @ v \mathrm{vr}=55 \mathrm{~V}$ & 7.46 & 6.76 & 0.7 & 1.51 & 0.53 & 0.98 \\
\hline $\mathrm{VF}(\mathrm{mV}) @ \mathrm{IF}=1 \mathrm{~mA}$ & 410.73 & 409.81 & 0.92 & 2.01 & 1.95 & 0.06 \\
\hline$V F(\mathrm{mV}) @ I F=10 \mathrm{~mA}$ & 578.71 & 577.75 & 0.96 & 1.59 & 1.74 & -0.15 \\
\hline $\operatorname{IR}(\mathrm{nA}) @ \mathrm{VR}=55 \mathrm{~V}$ & 6.78 & 6.75 & 0.03 & 1.04 & 0.55 & 0.49 \\
\hline$V F(m V) @ I F=1 \mathrm{~mA}$ & 411.8 & 410.29 & 1.51 & 2.29 & 1.89 & 0.4 \\
\hline$\overline{V F}(\mathrm{mV}) @ \mathrm{IF}=10 \mathrm{~mA}$ & 578.82 & 577.9 & 0.92 & 1.21 & 1.55 & -0.34 \\
\hline $\mathrm{IR}(\mathrm{pA}) @ \mathrm{VR}=10 \mathrm{~V}$ & 0.48 & 0.3 & 0.18 & 0.2 & 0.06 & 0.14 \\
\hline $\mathrm{IR}(\mathrm{pA}) @ \mathrm{VR}=30 \mathrm{~V}$ & 1.5 & 0.85 & 0.65 & 0.67 & 0.18 & 0.49 \\
\hline $\mathrm{VF}(\mathrm{mV}) @ \mathrm{IF}=1 \mathrm{~mA}$ & 577.24 & 576.85 & 0.39 & 1.21 & 1.57 & -0.36 \\
\hline$V F(\mathrm{mV}) @ I F=10 \mathrm{~mA}$ & 677.57 & 677.16 & 0.41 & 1.15 & 3.01 & .1 .86 \\
\hline $\mathrm{IR}(\mathrm{pA}) @ \mathrm{VR}=10 \mathrm{~V}$ & 1.1 & 0.35 & 0.75 & 0.74 & 0.25 & 0.49 \\
\hline $\mathrm{IR}(\mathrm{pA}) @ \mathrm{VR}=30 \mathrm{~V}$ & 3.3 & 1 & 2.3 & 2.3 & 0.78 & 1.52 \\
\hline$V F(m V) @ I F=1 \mathrm{~mA}$ & 577.42 & 576.76 & 0.66 & 1.2 & 1.57 & -0.37 \\
\hline $\mathrm{VF}(\mathrm{mV}) @ \mathrm{IF}=10 \mathrm{~mA}$ & 678.22 & 677.7 & 0.52 & 1.14 & 1.59 & -0.45 \\
\hline
\end{tabular}




\begin{tabular}{|c|c|c|c|c|c|c|}
\hline \multirow[t]{2}{*}{ TEST } & \multicolumn{3}{|c|}{ MINIMUM VALUES } & \multicolumn{3}{|c|}{ MAXIMUM VALUES } \\
\hline & Frean & DI Water & Delta & Freon & DI Wator & Delta \\
\hline $\mathrm{IR}(\mathrm{nA}) @ V R=55 \mathrm{~V}$ & 6.2 & 7.3 & -1.1 & 12 & 12 & 0 \\
\hline $\mathrm{VF}(\mathrm{mV}) @ \mathrm{IF}=1 \mathrm{~mA}$ & 616 & 614 & 2 & $63 !$ & 631 & 0 \\
\hline$V F(m V) @ I F=10 m A$ & 743.5 & 740 & 3.5 & 761.5 & 763 & -1.5 \\
\hline$\overline{I C B O}(\mathrm{nA}) @ \mathrm{VCB}=-50 \mathrm{~V}$ & 0.71 & 0.78 & 0.07 & 0.87 & 0,82 & 0.05 \\
\hline $\mathrm{ICBO}(\mathrm{nA}) @ \mathrm{VCB}=-70 \mathrm{~V}$ & 0.7 & 0.75 & -0.05 & 0.85 & 0.8 & 0.05 \\
\hline ICES $(\mathrm{nA}) @ V C E=-50 \mathrm{~V}$ & 0.45 & 0.44 & 0.01 & 0.6 & 0,48 & 0.12 \\
\hline $\operatorname{ICER}(\mathrm{nA}) @ \mathrm{VC}=-50 \mathrm{~V}, \mathrm{R}=1 \mathrm{~K}$ & 0,37 & 0,34 & 0.03 & 0,48 & 0.4 & 0.08 \\
\hline IEBO $(\mathrm{nA}) @ V E B=-5 \mathrm{~V}$ & 0.04 & 0.01 & 0.03 & 0.08 & 0.01 & 0.07 \\
\hline $\begin{array}{l}\text { VCES }(\mathrm{mV}) @ \mathrm{IC}=-10 \mathrm{~mA}, \mathrm{IB}=n \\
500 \mu \mathrm{A}\end{array}$ & 79.5 & 83 & -3.5 & 83.5 & 85 & -1.5 \\
\hline $\mathrm{VBES}(\mathrm{mV}) @ \mathrm{IC}=-10 \mathrm{~mA}, \mathrm{IB}=-500 \mu \mathrm{A}$ & 761.5 & 764 & -2.5 & 768 & 768 & 0 \\
\hline VBEON $(\mathrm{mV}) @ \mathrm{VCE}=.5 \mathrm{~V}, \mathrm{IC}=-5 \mathrm{~mA}$ & 700.5 & 700 & 0.5 & 708.5 & 704 & 4.5 \\
\hline VBEON $(\mathrm{mV}) @ \mathrm{VCE}=-5 \mathrm{~V}, \mathrm{IC}=-20 \mathrm{~mA}$ & 744 & 743 & 1 & 751.5 & 747 & 4.5 \\
\hline $1+\mathrm{HFE}$ Q $\mathrm{VCB}=-5 \mathrm{~V}, \mathrm{IE}=-10 \mu \mathrm{A}$ & 225 & 257 & -32 & 246 & 273 & -27 \\
\hline $\mathrm{HPE}$ ( ) VCE $=-5 \mathrm{~V}, \mathrm{IC}=-500 \mu \mathrm{A}$ & 219 & 249 & -30 & 239 & 264 & .25 \\
\hline $\mathrm{HFE} @ \mathrm{VCE}=-5 \mathrm{~V}, \mathrm{IC}=-10 \mathrm{~mA}$ & 206 & 233 & .27 & 223 & 247 & -24 \\
\hline HFE @ VCE $=-5 \mathrm{~V}, \mathrm{IC}=-20 \mathrm{~mA}$ & 193 & 218 & .25 & 209 & 231 & -22 \\
\hline $\mathrm{ICBO}(\mathrm{nA}) @ \mathrm{VCB}=20 \mathrm{~V}$ & 2 & 1.9 & 0.1 & 5 & 3.5 & 1.5 \\
\hline $\mathrm{IEBO}(\mathrm{nA}) @ \mathrm{VEB}=4 \mathrm{~V}$ & 12 & 12 & 0 & 146 & 178 & -32 \\
\hline ICER $(\mathrm{nIA}) @ \mathrm{VCE}=13 \mathrm{~V}, \mathrm{R}=1 \mathrm{~K}$ & 1.8 & 1.7 & 0.1 & 2.7 & 2.1 & 0.6 \\
\hline $\mathrm{HFE} @ \mathrm{VCE}=5 \mathrm{~V}, \mathrm{IC}=1 \mathrm{~mA}$ & 51.6 & 54.2 & .2 .6 & 109.2 & 89.8 & 19.4 \\
\hline $\mathrm{HFE} @ \mathrm{VCE}=5 \mathrm{~V}, \mathrm{IC}=10 \mathrm{~mA}$ & 70.1 & 71.3 & -1.2 & 133.7 & 113.6 & 20.1 \\
\hline $\mathrm{HFE}$ (2) VCE $=5 \mathrm{~V}, \mathrm{IC}=100 \mathrm{~mA}$ & 54.2 & 55.6 & -1.4 & 95.4 & 82.8 & 12.6 \\
\hline$V C E S(m V) @ I C=10 \mathrm{~mA}, I B=1 \mathrm{~mA}$ & 135.5 & 137.5 & -2 & 148.5 & 152 & -3.5 \\
\hline VBES $(\mathrm{mV}) @ \mathrm{IC}=10 \mathrm{~mA}, \mathrm{IB}=1 \mathrm{~mA}$ & 798 & 799.5 & -1.5 & 808 & 807.5 & 0.5 \\
\hline$\overline{V C E S}(\mathrm{mV}) @ I C=100 \mathrm{~mA}, I \mathrm{~B}=10 \mathrm{~mA}$ & 415.5 & 427 & -11.5 & 498 & 519 & .21 \\
\hline VBES $(\mathrm{mV}) @ \mathrm{IC}=100 \mathrm{~mA}, \mathrm{IB}=10 \mathrm{~mA}$ & 1119 & 1113.5 & 5.5 & 1154.5 & 1195.5 & -41 \\
\hline VBEON $(\mathrm{mV}) @ \mathrm{VCE}=5 \mathrm{~V}, \mathrm{IC}=5 \mathrm{~mA}$ & 717.5 & 718 & -0.5 & 733.5 & 731 & 2.5 \\
\hline VBEON $(\mathrm{mV}) @ \mathrm{VCE}=5 \mathrm{~V}, I \mathrm{C}=10 \mathrm{~mA}$ & 768.5 & 772 & .3 .5 & 791.5 & 788 & 3.5 \\
\hline $\mathrm{ICBO}(\mathrm{nA}) @ \mathrm{VCB}=-50 \mathrm{~V}$ & 0.48 & 0.59 & -0.11 & 2.8 & 0,62 & 2.18 \\
\hline $\mathrm{ICBO}(\mathrm{nA}) @ \mathrm{VCB}=-70 \mathrm{~V}$ & 0.55 & 0.6 & -0.05 & 2.8 & 0.7 & 2.1 \\
\hline ICES $(\mathrm{nA}) @ \mathrm{VCE}=-50 \mathrm{~V}$ & 0.075 & 0.2 & -0.125 & 2.4 & 0.25 & 2.15 \\
\hline$I C E R(n A) @ V C=-50 V, R=1 K$ & 0.18 & 0.28 & -0.1 & 2.5 & 0.32 & 2.18 \\
\hline $\operatorname{IEBO}(n A) @ V E B=-5 V$ & 0.01 & 0.01 & 0 & 1.8 & 0.01 & 1.79 \\
\hline$\overline{V C E S}(\mathrm{mV}) @ \mathrm{IC}=-10 \mathrm{~mA}, I \mathrm{IB}=-500 \mu \mathrm{A}$ & 79.5 & 83 & -3.5 & 83 & 84.5 & -1.5 \\
\hline VBES $(\mathrm{mV}) @ \mathrm{IC}=-10 \mathrm{~mA}, \mathrm{IB}=.500 \mu \mathrm{A}$ & 762 & 764 & -2 & 768.5 & 767.5 & 1 \\
\hline VBEON $(\mathrm{mV}) @ \mathrm{VCE}=-5 \mathrm{~V}, I \mathrm{C}=-5 \mathrm{~mA}$ & 701 & 700 & 1 & 709 & 704.5 & 4.5 \\
\hline VBEON $(\mathrm{mV}) @ \mathrm{VCE}=-5 \mathrm{~V}, \mathrm{IC}=-20 \mathrm{~mA}$ & 744 & 743 & 1 & 751.5 & 747 & 4.5 \\
\hline $1+\mathrm{HFE} @ \mathrm{VCB}=-5 \mathrm{~V}, \mathrm{IE}=-10 \mu \mathrm{A}$ & 224 & 254 & -30 & 256 & 271 & -15 \\
\hline HFE @ VCE $=-5 V, I C=-500 \mu \mathrm{A}$ & 219 & 246 & -27 & 239 & 263 & .24 \\
\hline $\mathrm{HFE} @ \mathrm{VCE}=-5 \mathrm{~V}, \mathrm{IC}=-10 \mathrm{~mA}$ & 206 & 230 & -24 & 223 & 245 & -22 \\
\hline $\mathrm{HFE} @ \mathrm{VCE}=-5 \mathrm{~V}, \mathrm{IC}=-20 \mathrm{~mA}$ & 192.7 & 215 & .22 .3 & 209 & 229 & .20 \\
\hline $\operatorname{IR}(\mathrm{nA}) @ \mathrm{VR}=55 \mathrm{~V}$ & 6.4 & 7.3 & -0.9 & 28 & 11 & 17 \\
\hline$V F(m V) @ I F=1 \mathrm{~mA}$ & 620.5 & 617.5 & 3 & 632 & 631 & 1 \\
\hline $\mathrm{VF}(\mathrm{mV}) @ \mathrm{IF}=10 \mathrm{~mA}$ & 747.5 & 745.5 & 2 & 761.5 & 761.5 & 0 \\
\hline $\mathrm{ICBO}(\mathrm{nA}) @ \mathrm{VCB}=-50 \mathrm{~V}$ & 0.68 & 0.76 & -0.08 & 0.82 & 0.79 & 0.03 \\
\hline $\mathrm{ICBO}(\mathrm{nA}) @ \mathrm{VCB}=-70 \mathrm{~V}$ & 0.65 & 0.65 & 0 & 0.85 & 0.8 & 0.05 \\
\hline $\operatorname{ICES}(n \mathrm{~A}) @ \mathrm{VCE}=-50 \mathrm{~V}$ & 0.42 & 0.44 & -0.02 & 0.56 & 0.48 & 0.08 \\
\hline $\operatorname{ICER}(\mathrm{nA}) @ \mathrm{VC}=-50 \mathrm{~V}, \mathrm{R}=1 \mathrm{~K}$ & 0.32 & 0.34 & -0.02 & 0.46 & 0.4 & 0.06 \\
\hline IFBO $(\mathrm{nA}) @$ VEB $=-5 \mathrm{~V}$ & 0.01 & 0.01 & 0 & 0.06 & 0.04 & 0.02 \\
\hline $\begin{array}{l}\text { VCES }(\mathrm{mV}) @ \mathrm{IC}=-10 \mathrm{~mA}, \mathrm{IB}= \\
500 \mu \mathrm{A}\end{array}$ & 79.5 & 83.5 & -4 & 82 & 86 & -4 \\
\hline VBES $(\mathrm{mV}) @ I \mathrm{IC}=-10 \mathrm{~mA}, \mathrm{IB}=-500 \mu \mathrm{A}$ & 762 & 764 & -2 & 769 & 767 & 2 \\
\hline
\end{tabular}




\begin{tabular}{|c|c|c|c|c|c|c|}
\hline \multirow[t]{2}{*}{ TEST } & \multicolumn{3}{|c|}{ MINIMUM VALUES } & \multicolumn{3}{|c|}{ MAXIMUM VALUES } \\
\hline & Freon & DI Water & Dolta & Freon & DI Water & Delta \\
\hline VBEON $(\mathrm{mV}) @ \mathrm{VCE}=-5 \mathrm{~V}, \mathrm{IC}=-20 \mathrm{~mA}$ & 744 & 743 & 1 & 752 & 747 & 5 \\
\hline VBEON $(\mathrm{mV}) @ V C E=-5 \mathrm{~V}, I C=-5 \mathrm{~mA}$ & 701 & 700 & 1 & 710 & 704 & 6 \\
\hline $1+\mathrm{HFE} @ \mathrm{VCB}=-5 \mathrm{~V}, \mathrm{IE}=.10 \mu \mathrm{A}$ & 225 & 253 & .28 & 246 & 274 & .28 \\
\hline HFE @ VCE $=.5 \mathrm{~V}, I \mathrm{C}=.500 \mu \mathrm{A}$ & 219 & 245 & .26 & 239 & 265 & -26 \\
\hline HFE @ VCE $=-5 \mathrm{~V}, I \mathrm{C}=-10 \mathrm{~mA}$ & .206 & 229 & -23 & 223 & 248 & .25 \\
\hline $\mathrm{HFE} @ \mathrm{VCE}=-5 \mathrm{~V}, I \mathrm{C}=-20 \mathrm{~mA}$ & 192.5 & 214 & -21.5 & 209 & 232 & .23 \\
\hline$I C B O(n A) @ V C B=20 V$ & 1.9 & 1.9 & 0 & 4.4 & 5.7 & -1.3 \\
\hline IEBO (nA) $(9) V E B=4 V$ & 12 & 12 & 0 & 212 & 165 & 47 \\
\hline ICER (nA) Q VCE $=13 V, R=1 \mathrm{~K}$ & 1.5 & 1.7 & .0 .2 & 2.3 & 2.2 & 91 \\
\hline $\mathrm{HFE} @ V C E=5 \mathrm{~V}, I \mathrm{C}=1 \mathrm{~mA}$ & 35.7 & 56 & .0 .3 & 129.5 & 94.6 & 34,9 \\
\hline HFE @ VCE $=5 \mathrm{~V}, I \mathrm{C}=10 \mathrm{~mA}$ & 43.5 & 73,4 & 0.1 & 153,6 & 119.3 & 34,3 \\
\hline HFE @VCE $=5 \mathrm{~V}, \mathrm{IC}=100 \mathrm{~mA}$ & 56.1 & 56.6 & -0.5 & 105 & 86.5 & 18.5 \\
\hline $\operatorname{VCES}(\mathrm{mV}) @ \mathrm{IC}=10 \mathrm{~mA}, \mathrm{IB}=1 \mathrm{~mA}$ & 131.5 & 138 & .6 .5 & 147.5 & 152 & -4.5 \\
\hline VBES $(m \mathrm{~m}) @ I C=10 \mathrm{~mA}, I B=1 \mathrm{~mA}$ & 796 & 799.5 & -3.5 & 807.5 & 806 & 1.5 \\
\hline VCES $(\mathrm{mV}) @ I C=100 \mathrm{~mA}, I \mathrm{~B}=10 \mathrm{~mA}$ & 410 & 436.5 & $.26,5$ & 498.5 & 535 & .36 .5 \\
\hline VBES $(m \mathrm{~V}) @ I \mathrm{C}=100 \mathrm{~mA}, I B=10 \mathrm{~mA}$ & 1112.5 & 1112.5 & 0 & 1167 & 1177 & -10 \\
\hline $\mathrm{VBEON}(\mathrm{mV}) @ \mathrm{VCE}=5 \mathrm{~V}, \mathrm{IC}=5 \mathrm{~mA}$ & 713.5 & 716.5 & -3 & 732 & 731 & 1 \\
\hline VBEON $(\mathrm{mV}) @ V C E=5 \mathrm{~V}, I \mathrm{C}=20 \mathrm{~mA}$ & 764,5 & 770.5 & -6 & 788.5 & 788 & 0.5 \\
\hline ICBO $(n A) @ V C B=-50 V$ & 0.72 & 0.63 & 0.09 & 0.77 & 0.66 & 0.11 \\
\hline $\mathrm{ICBO}(\mathrm{nA}) @ V \mathrm{VB}=-70 \mathrm{~V}$ & 0.75 & 0.65 & 0.1 & 0,9 & 0.8 & 0.1 \\
\hline $\mathrm{ICES}(\mathrm{nA}) @ \mathrm{VCE}=.50 \mathrm{~V}$ & 0.32 & 0.28 & 0.04 & 0.46 & 0.32 & 0.14 \\
\hline $\operatorname{ICER}(\mathrm{nA}) @ V C=-50 \mathrm{~V}, \mathrm{R}=1 \mathrm{~K}$ & 0.4 & 0.32 & 0,08 & 0.44 & 0.36 & 0.08 \\
\hline IEBO (nA)@VEB=-5V & 0.01 & 0.01 & 0 & 0.01 & 0.01 & 0 \\
\hline $\operatorname{VCES}(\mathrm{mV}) @ \mathrm{IC}=-10 \mathrm{~mA}, \mathrm{~B}=-500 \mu \mathrm{A}$ & 79.5 & 83 & -3.5 & 83 & 86 & -3 \\
\hline $\mathrm{VBES}(\mathrm{mV}) @ I C=-10 \mathrm{~mA}, \mathrm{IB}=-500 \mu \mathrm{A}$ & 762 & 764 & -2 & 768.5 & 767 & 1.5 \\
\hline $\mathrm{VBEON}(\mathrm{mV}) @ \mathrm{VCE}=-5 \mathrm{~V}, \mathrm{IC}=-5 \mathrm{~mA}$ & 702 & 700 & 2 & 709.6 & 704 & 5.6 \\
\hline $\mathrm{VBEON}(\mathrm{mV}) @ \mathrm{VCE}=-5 \mathrm{~V}, \mathrm{IC}=-20 \mathrm{~mA}$ & 744.5 & 743 & 1.5 & 751.5 & 747 & 4.5 \\
\hline $1+\mathrm{HFE} @ \mathrm{VCB}=-5 \mathrm{~V}, \mathrm{IE}=-10 \mu \mathrm{A}$ & 225 & 255 & -30 & 245 & 272 & -27 \\
\hline HFE @ VCE $=-5 \mathrm{~V}, I \mathrm{C}=-500 \mu \mathrm{A}$ & 219 & 247 & -28 & 238 & 264 & .26 \\
\hline HFE @ VCE $=-5 \mathrm{~V}, \mathrm{IC}=-10 \mathrm{~mA}$ & 206 & 230 & .24 & 222 & 247 & -25 \\
\hline $\mathrm{HFE} @ V C E=-5 \mathrm{~V}, \mathrm{IC}=-20 \mathrm{~mA}$ & 192.9 & 215 & -22.1 & 208 & 231 & -23 \\
\hline
\end{tabular}


TABLE A26: AVGERAGE AND STANDARD DEVIDATION OF INITIAL ELECTRICAL TEST FOR M-50 TEST

AVERAGE VALUES

\begin{tabular}{|c|c|c|c|c|c|c|}
\hline TEST & \multicolumn{3}{|c|}{ AVERAGE VALUES } & \multicolumn{3}{|c|}{ STANDARD DEVIATION } \\
\hline & Preon & DI Water & Dolta & Freon & DI Water & Delta \\
\hline $\mathrm{IR}(\mathrm{nA}) \otimes \mathrm{VR}=55 \mathrm{~V}$ & 9.7 & 9.7 & 0 & 1.1 & 1.1 & 0 \\
\hline$V F(m V) @ I F=1 \mathrm{~mA}$ & 623,94 & 625.45 & -1.51 & 2.91 & 3.24 & .0 .33 \\
\hline $\mathrm{VF}(\mathrm{nV}) @ \mathrm{IF}=10 \mathrm{~mA}$ & 753.16 & 755.48 & .2 .32 & 4.24 & 4,36 & $-0,12$ \\
\hline$I C B O(n A) @ V C B=-50 V$ & 0.81 & 0.79 & 0.02 & 0.03 .5 & 0,005 & 0,03 \\
\hline$I C B O(n A) @ V C B=-70 V$ & 0.8 & 0.78 & 0.02 & 0.031 & 0,025 & 0.006 \\
\hline ICES(nA)@VCE $=-50 V$ & 0.51 & 0.46 & 0.05 & 0.024 & 0.014 & 0.01 \\
\hline $\operatorname{ICER}(\mathrm{nA}) \mathrm{VC}=-50 \mathrm{~V}, \mathrm{R}=1 \mathrm{~K}$ & 0.41 & 0.36 & 0.05 & 0,015 & 0.014 & 0.001 \\
\hline $1 E B O(n A) @ V E B=-5 V$ & 0,054 & 0,0052 & 0.0488 & 0.014 & 0.0009 & 0,0131 \\
\hline $\begin{array}{l}\text { VCES }(\mathrm{mV}) @ \mathrm{IC}=-10 \mathrm{~mA}, \overline{\mathrm{IB}}=\ldots \\
500 \mu \mathrm{A}\end{array}$ & 80.9 & 83.95 & -3.05 & 1 & 0.416 & 0,584 \\
\hline VBES $(m V) @ I C=-10 m A, I B=-500 \mu A$ & 763.81 & 765.23 & -1.42 & 1.58 & $1 . \overline{15}$ & 0.43 \\
\hline VBEON $(\mathrm{mV}) @ V C E=-5 \mathrm{~V}, I \mathrm{C}=-5 \mathrm{~mA}$ & 703.29 & 701.92 & 1.37 & 1.77 & 1.45 & 0.32 \\
\hline VBEON $(\mathrm{mV}) @ V C E=-5 \mathrm{~V}, \mathrm{IC}=-20 \mathrm{~mA}$ & 746.13 & 744,68 & 1.45 & 1.7 & 1.17 & 0,53 \\
\hline $1+\mathrm{HFE} @ \mathrm{VCB}=-5 \mathrm{~V}, \mathrm{IE}=-10 \mu \mathrm{A}$ & 235.11 & 265.58 & .30 .47 & 5.5389 & 3,8763 & 1.6626 \\
\hline HFE @ VCE $=-5 \mathrm{~V}, I \mathrm{C}=-500 \mu \mathrm{A}$ & 228.41 & 256.93 & -28.52 & 5.0732 & 3.5531 & 1.5201 \\
\hline $\mathrm{HFE} @ \mathrm{VCE}=-5 \mathrm{~V}, \mathrm{IC}=-10 \mathrm{~mA}$ & 213.79 & 240.24 & -26.45 & 4,4219 & 3.2884 & 11335 \\
\hline HFE @ VCE $=-5 V, I C=-20 \mathrm{~mA}$ & 200.34 & 224.73 & $-24,39$ & 4.1062 & 3.0274 & 1,0788 \\
\hline $\mathrm{ICBO}(\mathrm{nA}) @ V C B=20 \mathrm{~V}$ & 2.8 & 2.4 & 0.4 & 0.61 & 0.25 & 0.36 \\
\hline IEBO $(\mathrm{nA}) @ \mathrm{VEB}=4 \mathrm{~V}$ & 28 & 19 & 9 & 29 & 23 & 6 \\
\hline $\operatorname{ICER}(\mathrm{nA}) @ V C E=13 \mathrm{~V}, \mathrm{R}=1 \mathrm{~K}$ & 2.2 & 1.8 & 0,4 & 0.16 & 0.1 & 0.06 \\
\hline HFE @ VCE $=5 \mathrm{~V}, I \mathrm{C}=1 \mathrm{~mA}$ & 71.014 & 75.209 & -4.195 & 15,806 & 9.5693 & 6.2367 \\
\hline HFE @ VCE $=5 \mathrm{~V}, I \mathrm{C}=10 \mathrm{~mA}$ & 91.455 & 97.445 & $-5,99$ & 18.255 & 11.303 & 6.952 \\
\hline HFE @ VCE $=5 \mathrm{~V}, I \mathrm{C}=100 \mathrm{~mA}$ & 67,98 & 73.14 & $-5,16$ & 11,77 & 7,3149 & 4,4551 \\
\hline$V C E S(m V) @ I C=10 \mathrm{~mA}, I B=1 \mathrm{~mA}$ & 144,38 & 142,49 & 1.89 & 4.02 & 4 & 0.02 \\
\hline VBES (InV)@IC $=10 \mathrm{~mA}, I B=1 \mathrm{~mA}$ & 802.61 & 802.61 & 0 & 2.2 & 1.84 & 0.36 \\
\hline $\mathrm{VCES}(\mathrm{mV})(\mathrm{IC}=100 \mathrm{~mA}, \mathrm{IB}=10 \mathrm{~mA}$ & 470,33 & 469.55 & 0,78 & 26.32 & 20.58 & 5.74 \\
\hline VBES $(\mathrm{mV}) @ I C=100 \mathrm{~mA}, I B=10 \mathrm{~mA}$ & 1133.9 & 1144.1 & -10.2 & 8.73 & 19.78 & -11.05 \\
\hline VBEON $(\mathrm{mV}) @$ VCE $=5 \mathrm{~V}, \mathrm{IC}=5 \mathrm{~mA}$ & 725.15 & 722.48 & 2.67 & 3.34 & 3.18 & 0.16 \\
\hline $\operatorname{VBEON}(\mathrm{mV}) @ \mathrm{VCE}=5 \mathrm{~V}, I \mathrm{C}=10 \mathrm{~mA}$ & 780.64 & 777.31 & 3.33 & 5.05 & 4.01 & 1.04 \\
\hline $\mathrm{ICBO}(\mathrm{nA}) @ \mathrm{VCB}=-50 \mathrm{~V}$ & 0.61 & 0.6 & 0.01 & 0.3 & 0.0065 & 0.2935 \\
\hline $1 \mathrm{CBO}(\mathrm{nA}) @ V \mathrm{VB}=-70 \mathrm{~V}$ & 0.69 & 0.66 & 0.03 & 0.29 & 0.004 & 0.286 \\
\hline$I C E S(n A) @ V C E=-50 \mathrm{~V}$ & 0,22 & 0.23 & -0.01 & 0.3 & 0.013 & 0.287 \\
\hline $\operatorname{ICER}(\mathrm{nA}) @ V C=.50 \mathrm{~V}, \mathrm{R}=1 \mathrm{~K}$ & 0.32 & 0.31 & 0.01 & 0.3 & 0.008 & 0.292 \\
\hline $\mathrm{IEBO}(\mathrm{nA}) @ V E B=-5 \mathrm{~V}$ & 0.037 & 0,005 & 0.032 & 0.24 & 0 & 0.24 \\
\hline $\operatorname{VCES}(\mathrm{mV}) @ \mathrm{IC}=-10 \mathrm{~mA}, \mathrm{IB}=-500 \mu \mathrm{A}$ & 80.82 & 83.93 & -3.11 & 0.783 & 0.413 & 0.37 \\
\hline VBES $(m V) @ I C=.10 m A, I B=-500 \mu A$ & 763.95 & 765.24 & -1.29 & 1.51 & 1,1 & 0.41 \\
\hline VBEON $(\mathrm{mV}) @ \mathrm{VCE}=-5 \mathrm{~V}, \mathrm{IC}=-5 \mathrm{~mA}$ & 703.5 & 701.97 & 1.53 & 1.85 & 1.44 & 0.41 \\
\hline VBEON $(m V) @ V C E=-5 V, I C=-20 m A$ & 746.31 & 744.76 & 1.55 & 1,83 & 1.13 & 0.7 \\
\hline $1+\mathrm{HFE} @ \mathrm{VCB}=-5 \mathrm{~V}, \mathrm{IE}=-10 \mu \mathrm{A}$ & 234.46 & 263.49 & -29.03 & 6.4497 & 4.2682 & 2.1815 \\
\hline $\mathrm{HFE} @ \mathrm{VCE}=-5 \mathrm{~V}, \mathrm{IC}=-500 \mu \mathrm{A}$ & 227.86 & 255.6 & -27.74 & 5.3477 & 3.8132 & 1.5345 \\
\hline HFE @ $V^{\prime} C E=-5 \mathrm{~V}, \mathrm{IC}=-10 \mathrm{~mA}$ & 213.34 & 239.02 & -25.68 & 4.7684 & 3.5875 & 1.1809 \\
\hline HFE @VCE $=-5 V, I C=-20 \mathrm{~mA}$ & 199.94 & 223.64 & -23.7 & 4.375 & 3.3019 & 1.0731 \\
\hline $\mathrm{IR}(\mathrm{nA}) @ \mathrm{VR}=55 \mathrm{~V}$ & 9.6 & 9.2 & 0.4 & 2.8 & 0.87 & 1.93 \\
\hline$V F(m V) @ I F=1 \mathrm{~mA}$ & 625.75 & 626.85 & $.1,1$ & 2.42 & 2.68 & -0.26 \\
\hline $\mathrm{VF}(\mathrm{mV}) @ \mathrm{IF}=10 \mathrm{~mA}$ & 755.7 & 757.2 & -1.5 & 3.31 & 3.71 & -0.4 \\
\hline$I C B O(n A) @ V C B=-50 V$ & 0.77 & 0.77 & 0 & 0.038 & 0.0077 & 0.0303 \\
\hline $\mathrm{ICBO}(\mathrm{nA}) @ \mathrm{VCB}=-70 \mathrm{~V}$ & 0.78 & 0.75 & 0.03 & 0.046 & 0.044 & 0,002 \\
\hline ICES (niA) @ VCE $=-50 \mathrm{~V}$ & 0.5 & 0.47 & 0.03 & 0.027 & 0.0078 & 0.0192 \\
\hline $\operatorname{ICER}(n \mathrm{~A}) @ V C=-50 V, R=1 \mathrm{~K}$ & 0.4 & 0.36 & 0.04 & 0.03 & 0.015 & 0.015 \\
\hline IEBO $(n A) @ V E B=-5 V$ & 0.034 & 0.0062 & 0.0278 & 0.014 & 0.0054 & 0.0086 \\
\hline $\begin{array}{l}\text { VCES }(\mathrm{mV}) @ I C=-10 \mathrm{~mA}, \mathrm{IR}=. \\
500 \mu \mathrm{A}\end{array}$ & 80.8 & 84.12 & .3 .32 & 0.724 & 0.49 & 0.234 \\
\hline VBES $(m V) @ I C=-10 m A, I B=-500 \mu A$ & 964.05 & 765.17 & 198.88 & 1.54 & 0.992 & 0.548 \\
\hline
\end{tabular}




\begin{tabular}{|c|c|c|c|c|c|c|}
\hline \multicolumn{7}{|c|}{$\begin{array}{l}\text { TABLE A26: A.VERAGE AND STANDARD IIIV/ATION OF INITIAL ELECTRICAL TEST FOR M-50 } \\
\text { (CONTINUED) }\end{array}$} \\
\hline \multirow[t]{2}{*}{ TEST } & \multicolumn{3}{|c|}{ AVERAGE VALUES } & \multicolumn{3}{|c|}{ STANDARD DEVIATION } \\
\hline & Freon & DI Water & Delta & Freon & DI Water & Delta \\
\hline VBEON $(m \mathrm{~V}) @ V C E=-5 \mathrm{~V}, \mathrm{IC}=-5 \mathrm{~mA}$ & 703,46 & 701.98 & 1.48 & 1.89 & 1.42 & 0.47 \\
\hline $\operatorname{VBEON}(\mathrm{mV}) @ V C E=-5 \mathrm{~V}, I \mathrm{C}=.20 \mathrm{~mA}$ & 746.21 & 744.75 & 1.46 & 1.79 & 1.12 & 0.67 \\
\hline $1+\mathrm{HFE} @ \mathrm{VCB}=.5 \mathrm{~V}, \mathrm{IE}=-10 \mu \mathrm{A}$ & 236.88 & 263.82 & $-26,94$ & 5,7463 & 4.8078 & 0.9385 \\
\hline $\mathrm{HFE} @ V \mathrm{VE}=.5 \mathrm{~V}, \mathrm{IC}=-500 \mu \mathrm{A}$ & 230.13 & 255.38 & -25.25 & 5,4608 & 4.4409 & 1.0199 \\
\hline $\mathrm{HFE} @ \mathrm{VCE}=-5 \mathrm{~V}, \mathrm{IC}=-10 \mathrm{~mA}$ & 215.27 & 238.73 & -23.46 & 4,6417 & 4.2751 & 0.3666 \\
\hline $\mathrm{HFE} @ V C E=-5 \mathrm{~V}, I \mathrm{C}=-20 \mathrm{~mA}$ & 201.58 & 223.22 & -21.64 & 4.3693 & 3.9379 & 0,4314 \\
\hline $\mathrm{ICBC}(\mathrm{nA}) @ \mathrm{VCB}=20 \mathrm{~V}$ & 2.7 & 2.7 & 0 & 0.48 & 0.81 & -0.33 \\
\hline $\operatorname{IEBO}(\mathrm{nA}) @ \mathrm{VEB}=4 \mathrm{~V}$ & 35 & 26 & 9 & 42 & 29 & 13 \\
\hline $\operatorname{ICER}(\mathrm{nA}) @ \mathrm{VCE}=13 \mathrm{~V}, \mathrm{R}=1 \mathrm{~K}$ & 2 & 1.9 & 0.1 & 0.17 & 0.12 & 0,05 \\
\hline $\mathrm{HFE} @ V C E=5 V, I C=1 \mathrm{~mA}$ & 91.209 & 65.247 & 25.962 & 22.944 & 9.2094 & 13.735 \\
\hline $\mathrm{HFE} @ V C E=5 \mathrm{~V}, I C=10 \mathrm{~mA}$ & 113.3 & 84.822 & 28.478 & 25.418 & 11.071 & 14,347 \\
\hline $\mathrm{HFE} @ \mathrm{VCE}=5 \mathrm{~V}, \mathrm{IC}=100 \mathrm{~mA}$ & 81.225 & 64.607 & 16.618 & 15.605 & 7.1912 & 8.4138 \\
\hline VCES $(\mathrm{mV}) @ \mathrm{IC}=10 \mathrm{~mA}, I \mathrm{IB}=1 \mathrm{~mA}$ & 138.79 & 147.65 & -8.86 & 4.43 & 3.73 & 0.7 \\
\hline VBES $(\mathrm{mV}) @ \mathrm{IC}=10 \mathrm{~mA}, \mathrm{IB}=1 \mathrm{~mA}$ & 801,56 & 802.55 & .0 .99 & 2.63 & 1.33 & 1.3 \\
\hline $\operatorname{VCES}(\mathrm{mV}) @ I \mathrm{C}=100 \mathrm{~mA}, I \mathrm{~B}=10 \mathrm{~mA}$ & 439.1 & 494.8 & -55.7 & 24.57 & 19.32 & 5.25 \\
\hline $\operatorname{VBES}(\mathrm{mV}) @ \mathrm{IC}=100 \mathrm{~mA}, \mathrm{IB}=10 \mathrm{~mA}$ & 1134.2 & 1125.2 & 9 & 12.39 & 13.4 & -1.01 \\
\hline $\mathrm{VBECN}(\mathrm{mV}) @ \mathrm{VCE}=5 \mathrm{~V}, \mathrm{IC}=5 \mathrm{~mA}$ & 722.38 & 72.6 .6 & $-4,22$ & 4,38 & 3.4 & 0.98 \\
\hline $\mathrm{VBEON}(\mathrm{mV}) @ \mathrm{VCE}=5 \mathrm{~V}, I \mathrm{C}=20 \mathrm{~mA}$ & 776.32 & 782.35 & -6.03 & 6.44 & 4.18 & 2.26 \\
\hline $\mathrm{ICBO}(\mathrm{nA}) @ \mathrm{VCB}=-50 \mathrm{~V}$ & 0.73 & 0.65 & 0.08 & 0.017 & 0.0064 & 0,0106 \\
\hline$I C B O(n A) @ V C B=-70 V$ & 0.81 & 0.75 & 0.06 & 0.03 & 0.051 & -0.021 \\
\hline ICES (nA)@VCE $=-50 \mathrm{~V}$ & 0.38 & 0.31 & 0.07 & 0.026 & 0.013 & 0.013 \\
\hline $\operatorname{ICER}(\mathrm{nA}) @ V C=-50 \mathrm{~V}, \mathrm{R}=1 \mathrm{~K}$ & 0.4 & 0.33 & 0.07 & 0.013 & 0.014 & -0.001 \\
\hline $\mathrm{IEBO}(\mathrm{nA}) @ \mathrm{VEB}=-5 \mathrm{~V}$ & 0.005 & 0.005 & 0 & 0 & 0 & 0 \\
\hline VCES $(\mathrm{mV}) @ \mathrm{IC}=-10 \mathrm{~mA}, \mathrm{IB}=-500 \mu \mathrm{A}$ & 80.76 & 84.55 & -3.79 & 0.792 & 0.571 & 0.221 \\
\hline $\mathrm{VBES}(\mathrm{mV}) @ \mathrm{IC}=-10 \mathrm{~mA}, \mathrm{IB}=-500 \mu \mathrm{A}$ & 764.1 & 765.35 & -1.25 & 1.42 & 1.12 & 0.3 \\
\hline VBEON $(m V) @ V C E=-5 V, I C=-5 m A$ & 703.48 & 702.02 & 1.46 & 1.77 & 1.41 & $0 . \overline{36}$ \\
\hline VBEON $(m V) @ V C E=-5 V, I C=-20 \mathrm{~mA}$ & 746.2 & 744.84 & 1.36 & 1.72 & 1.06 & 0.66 \\
\hline $1+\mathrm{HFE} @ \mathrm{VCB}=-5 \mathrm{~V}, \mathrm{IE}=-10 \mu \mathrm{A}$ & 237.3 & 264.22 & -26.92 & 4.9135 & 3.4838 & 1.4297 \\
\hline $\mathrm{HFE} @ \mathrm{VCE}=-5 \mathrm{~V}, \mathrm{IC}=-500 \mu \mathrm{A}$ & 230.68 & 255.84 & -25.16 & 4.6441 & 3.3097 & 1.3344 \\
\hline HFE@VCE $=-5 \mathrm{~V}, I \mathrm{C}=-10 \mathrm{~mA}$ & 215.66 & 239.31 & -23.65 & 3,9232 & 3.0844 & 0.8388 \\
\hline HFE @ VCE $=-5 \mathrm{~V}, \mathrm{IC}=-20 \mathrm{~mA}$ & 201.99 & 223.75 & -21.76 & 3.7006 & 2.9327 & 0.7679 \\
\hline
\end{tabular}




\begin{tabular}{|c|c|c|c|c|c|c|}
\hline \multirow[t]{2}{*}{ TEST } & \multicolumn{3}{|c|}{ MINIMUM VALUES } & \multicolumn{3}{|c|}{ MAXIMUM VALUES } \\
\hline & Freon & DI Water & Delta & Freon & DI Water & Delta \\
\hline $\mathrm{IR}(\mathrm{nA}) @ \mathrm{VR}=55 \mathrm{~V}$ & 9.5 & 13 & -3.5 & 16 & 21 & -5 \\
\hline $\mathrm{VF}(\mathrm{mV}) @ \mathrm{IF}=\operatorname{lmA}$ & 620 & 610 & 10 & $63 !$ & 627.5 & 3.5 \\
\hline $\mathrm{VF}(\mathrm{mV}) @ \mathrm{IF}=10 \mathrm{~mA}$ & 747 & 736 & 11 & 762 & 760 & 2 \\
\hline ICBO (nA) @ VCB $=-50 \mathrm{~V}$ & 1.9 & 0.71 & 1.19 & 2 & 0.79 & 1.21 \\
\hline $\mathrm{ICBO}(\mathrm{nA}) @ \mathrm{VCB}=-70 \mathrm{~V}$ & 1.8 & 0.65 & 1.15 & 2 & 0.8 & 1.2 \\
\hline $\operatorname{ICES}(n \mathrm{~A}) @ \mathrm{VCE}=-50 \mathrm{~V}$ & 1 & 0.38 & 0,62 & 1 & 0.47 & 0.53 \\
\hline$I C E R(n A) @ V C=-50 V, R=1 K$ & 0.76 & 0.3 & 0.46 & 0.8 & 0,36 & 0.44 \\
\hline $\mathrm{IEBO}(\mathrm{nA}) @ \mathrm{VEB}=-5 \mathrm{~V}$ & 0.01 & 0.01 & 0 & 0.01 & 0.01 & 0 \\
\hline $\begin{array}{l}\text { VCES }(\mathrm{mV}) @ \mathrm{IC}=-10 \mathrm{~mA}, \mathrm{IB}=\cdots \\
500 \mu \mathrm{A}\end{array}$ & 80 & 83.5 & -3.5 & 84 & 86 & -2 \\
\hline VBES (mV) @ IC $=-10 \mathrm{~mA}, I \mathrm{~B}=-500 \mu \mathrm{A}$ & 761.5 & 762 & -0.5 & 767 & 766 & 1 \\
\hline VBEON $(\mathrm{mV}) \Leftrightarrow \mathrm{VCE}=-5 \mathrm{~V}, 1 \mathrm{C}=-5 \mathrm{~mA}$ & 673.5 & 699 & -25.5 & 690 & 704 & .14 \\
\hline VBEON $(m V) @ V C E=-5 V, I C=-20 m A$ & 666.5 & 741.5 & .75 & 716 & 746.5 & -30.5 \\
\hline $1+\mathrm{HFE} @ \mathrm{VCB}=-5 \mathrm{~V}, \mathrm{IE}=-10 \mu \mathrm{A}$ & 221 & 253 & -32 & 239 & 266 & -27 \\
\hline HFE @ VCE $=-5 \mathrm{~V}, \mathrm{IC}=-500 \mu \mathrm{A}$ & 215 & 246 & .31 & 232 & 258 & -26 \\
\hline HFE @ VCE $=-5 \mathrm{~V}, \mathrm{IC}=-10 \mathrm{~mA}$ & 202 & 229 & -27 & 217 & 240 & -23 \\
\hline $\mathrm{HFE} @ \mathrm{VCE}=-5 \mathrm{~V}, \mathrm{IC}=-20 \mathrm{~mA}$ & 268 & 215 & 53 & 391 & 225 & 166 \\
\hline $\mathrm{ICBO}(\mathrm{nA}) @ \mathrm{VCB}=20 \mathrm{~V}$ & 2.8 & 2.9 & $-0,1$ & 4.7 & 4.7 & 0 \\
\hline IEBO (nA)@ VEB $=4 \mathrm{~V}$ & 13 & 13 & 0 & 131 & 106 & 25 \\
\hline $\operatorname{ICER}(\mathrm{nA}) @ \mathrm{VCE}=13 \mathrm{~V}, \mathrm{R}=1 \mathrm{~K}$ & 2.1 & 2,3 & -0.2 & 3.1 & 3.3 & -0.2 \\
\hline $\mathrm{HFE} @ \mathrm{VCE}=5 \mathrm{~V}, \mathrm{IC}=1 \mathrm{~mA}$ & 50,9 & 54,4 & .3 .5 & 108.8 & 90.4 & 18.4 \\
\hline $\mathrm{HFE} @ \mathrm{VCE}=5 \mathrm{~V}, \mathrm{IC}=10 \mathrm{~mA}$ & 69.2 & 71.4 & -2.2 & 133.2 & 114.8 & 18.4 \\
\hline $\mathrm{HFE} @ \mathrm{VCE}=5 \mathrm{~V}, \mathrm{IC}=100 \mathrm{~mA}$ & 53.7 & 55.6 & -1.9 & 95.8 & 83.6 & 12.2 \\
\hline VCES $(\mathrm{mV}) @ \mathrm{IC}=10 \mathrm{~mA}, \mathrm{IB}=1 \mathrm{~mA}$ & 135.5 & 139 & -3.5 & 148.5 & 153.5 & -5 \\
\hline VBES(mV)@IC $=10 \mathrm{~mA}, I \mathrm{~B}=1 \mathrm{~mA}$ & 798 & 797.5 & 0.5 & 807 & 804.5 & 2.5 \\
\hline VCES (mV)@IC $=100 \mathrm{~mA}, 1 \mathrm{~B}=10 \mathrm{~mA}$ & 416 & 428.5 & -12.5 & 498.5 & 521 & -22.5 \\
\hline VBES (mV)@IC $=100 \mathrm{~mA}, I B=10 \mathrm{~mA}$ & 1116.5 & 1111.5 & 5 & 1151 & 1179.5 & .28 .5 \\
\hline VBEON $(\mathrm{mV}) @ V C E=5 \mathrm{~V}, I \mathrm{C}=5 \mathrm{~mA}$ & 716 & 716 & 0 & 732 & 729.5 & 2.5 \\
\hline VBEON $(\mathrm{mV}) @ \mathrm{VCE}=5 \mathrm{~V}, I \mathrm{C}=10 \mathrm{~mA}$ & 768 & 769.5 & -1.5 & 790 & 787 & 3 \\
\hline$I \mathrm{CBO}(\mathrm{nA}) @ \mathrm{VCB}=-50 \mathrm{~V}$ & 1.5 & 0.52 & 0.98 & 1.5 & 0.59 & 0.91 \\
\hline$I C B O(n A) @ V C B=-70 V$ & 1.6 & 0.55 & 1.05 & 1.8 & 0.7 & 1.1 \\
\hline ICES (nA)@VCE $=-50 \mathrm{~V}$ & 0.68 & 0.31 & 0.37 & 0.88 & 0.36 & 0.52 \\
\hline $\operatorname{ICER}(n A) @ V C=-50 V, R=1 K$ & 0.65 & 0.24 & 0,41 & 0.72 & 0.32 & 0.4 \\
\hline IEBO $(n A) @ V E B=-5 V$ & 0.01 & 0.01 & 0 & 0.01 & 0.01 & 0 \\
\hline $\mathrm{VCES}(\mathrm{mV}) @ \mathrm{IC}=-10 \mathrm{~mA}, \mathrm{IB}=-500 \mu \mathrm{A}$ & 80.5 & 84.5 & -4 & 83.5 & 87 & -3.5 \\
\hline VBES $(\mathrm{mV}) @ \mathrm{IC}=-10 \mathrm{~mA}, \mathrm{IB}=-500 \mu \mathrm{A}$ & 761.5 & 762 & -0.5 & 766 & 768 & -2 \\
\hline VBEON $(m \mathrm{~V}) @ V C E=-5 \mathrm{~V}, \mathrm{IC}=-5 \mathrm{~mA}$ & 700.5 & 699 & 1.5 & 707.5 & 75.5 & 2 \\
\hline VBEON $(m \mathrm{~m}) @ \mathrm{VCE}=-5 \mathrm{~V}, \mathrm{IC}=-20 \mathrm{~mA}$ & 742 & 742 & 0 & 748 & 748 & 0 \\
\hline $1+\mathrm{HFE} @ \mathrm{VCB}=-5 \mathrm{~V}, \mathrm{IE}=-10 \mu \mathrm{A}$ & 213 & 242 & .29 & 237 & 256 & -19 \\
\hline $\mathrm{HFE} @ \mathrm{VCE}=-5 \mathrm{~V}, \mathrm{IC}=-500 \mu \mathrm{A}$ & 207 & 235 & -28 & 230 & 248 & -18 \\
\hline HFE@ $Q C E=-5 \mathrm{~V}, I \mathrm{C}=-10 \mathrm{~mA}$ & 193.4 & 219 & -25.6 & 216 & 231 & -15 \\
\hline HFE @ VCE $:-5 \mathrm{~V}, \mathrm{IC}=-20 \mathrm{~mA}$ & 181.7 & 206 & -24.3 & 202 & 216 & .14 \\
\hline $\mathrm{IR}(\mathrm{nA}) @ \mathrm{VR}=55 \mathrm{~V}$ & 8.5 & 12 & -3.5 & 15 & 20 & -5 \\
\hline $\mathrm{VF}(\mathrm{mV}) @ \mathrm{IF}=1 \mathrm{~mA}$ & 623.5 & 615.5 & 8 & 631 & 631.5 & -0.5 \\
\hline $\mathrm{VF}(\mathrm{mV}) @ \mathrm{IF}=10 \mathrm{~mA}$ & 749.5 & 743.5 & 6 & 761.5 & 763 & -1.5 \\
\hline $\mathrm{ICBO}(\mathrm{nA}) @ \mathrm{VCB}=-.50 \mathrm{~V}$ & 1.9 & 0.68 & 1.22 & 2 & 0.76 & 1.24 \\
\hline $\mathrm{ICBO}(\mathrm{nA}) @ \mathrm{VCB}=-70 \mathrm{~V}$ & 1.9 & 0.6 & 1.3 & 2 & 0.8 & 1.2 \\
\hline $\operatorname{ICES}(\mathrm{nA}) @ \mathrm{VCE}=-50 \mathrm{~V}$ & 1 & 0.39 & 0.61 & 1 & 0.48 & 0.52 \\
\hline $\operatorname{ICER}(\mathrm{nA}) @ \mathrm{VC}=-50 \mathrm{~V}, \mathrm{R}=1 \mathrm{~K}$ & 0.76 & 0.3 & 0.46 & 0.8 & 0.36 & 0.44 \\
\hline $\mathrm{IEBO}(\mathrm{nA}) @ \mathrm{VEB}=-5 \mathrm{~V}$ & 0.01 & 0.01 & 0 & 0.01 & 0.01 & 0 \\
\hline $\begin{array}{l}\mathrm{VCES}(\mathrm{mV}) @ \mathrm{IC}=-10 \mathrm{~mA}, \mathrm{IB}=- \\
500 \mu \mathrm{A}\end{array}$ & 80.5 & 84 & -3.5 & 83.5 & 87 & -3.5 \\
\hline VBES $(m V) @ I C=-10 m A, I B=-500 \mu A$ & 761.5 & 762 & -0.5 & 767 & 768 & -1 \\
\hline
\end{tabular}




\begin{tabular}{|c|c|c|c|c|c|c|}
\hline \multicolumn{7}{|c|}{$\begin{array}{l}\text { TABLE A27: MINIMUM AND MAXIMUM VALUES OF POST H }{ }^{*} \text { RB ELECTRICAL TEST DATA FOR M-50 } \\
\text { (CONTINUED) }\end{array}$} \\
\hline \multirow[t]{2}{*}{ TEST } & \multicolumn{3}{|c|}{ MINIMUM VALIJES } & \multicolumn{3}{|c|}{ MAXIMUM VALUES } \\
\hline & Freon & DI Water & Delta & Freon & DI Water & Delta \\
\hline VBEON $(m V) @ V C E=-5 V, I C=-5 m A$ & 700.5 & 699 & 1.5 & 707.5 & 705.5 & 2 \\
\hline VBEON (mV) @ VCE $=-5 V, I C=-20 \mathrm{~mA}$ & 737.5 & 742 & -4.5 & 744 & 748 & .4 \\
\hline $1+\mathrm{HFE} @ \mathrm{VCB}=-5 \mathrm{~V}, \mathrm{IE}=-10 \mu \mathrm{A}$ & 224 & 249 & -25 & 239 & 367 & -128 \\
\hline $\mathrm{HFE} @ \mathrm{VCE}=-5 \mathrm{~V}, \mathrm{IC}=-500 \mu \mathrm{A}$ & 217 & 242 & -25 & 232 & 258 & -26 \\
\hline HFE @ VCE $=-5 \mathrm{~V}, I \mathrm{IC}=-10 \mathrm{~mA}$ & 203 & 226 & -23 & 216 & 241 & -25 \\
\hline HFE @ VCE $=-5 \mathrm{~V}, \mathrm{IC}=-20 \mathrm{~mA}$ & 190.7 & 212 & -21.3 & 203 & 226 & -23 \\
\hline $\mathrm{ICBO}(\mathrm{nA}) @ \mathrm{VCB}=20 \mathrm{~V}$ & 2.5 & 2.7 & -0.2 & 4.3 & 6.3 & -2 \\
\hline IEBO $(\mathrm{nA}) @ \mathrm{VEB}=4 \mathrm{~V}$ & 12 & 13 & -1 & 234 & 141 & 93 \\
\hline $\operatorname{ICER}(\mathrm{nA}) \mathbb{Q}: Z E=13 \mathrm{~V}, \mathrm{R}=1 \mathrm{~K}$ & 1.9 & 2.3 & -0.4 & 3.1 & 3.1 & 0 \\
\hline HFE @ VCE $=5 \mathrm{~V}, \mathrm{IC}=1 \mathrm{~mA}$ & 54.7 & 56 & -1.3 & 127 & 94.7 & 32.3 \\
\hline $\mathrm{HFE} @ \mathrm{VCE}=5 \mathrm{~V}, \mathrm{IC}=10 \mathrm{~mA}$ & 72.3 & 73.4 & -1.1 & 150.9 & 119.4 & 31.5 \\
\hline HFE @ VCE $=5 \mathrm{~V}, \mathrm{IC}=100 \mathrm{~mA}$ & 55.2 & 56.8 & -1.6 & 103.4 & 86.5 & 16.9 \\
\hline VCES $(\mathrm{mV}) @ \mathrm{IC}=10 \mathrm{~mA}, \mathrm{IB}=1 \mathrm{~mA}$ & 131.5 & 139 & -7.5 & 147.5 & 152.5 & -5 \\
\hline VBES $(\mathrm{mV}) @ \mathrm{IC}=10 \mathrm{~mA}, \mathrm{IB}=\operatorname{lmA}$ & 798.5 & 798 & 0.5 & 806 & 805.5 & 0.5 \\
\hline $\mathrm{VCES}(\mathrm{mV}) @ \mathrm{IC}=100 \mathrm{~mA}, \mathrm{~KB}=10 \mathrm{~mA}$ & 410 & 438.5 & -28.5 & 499.5 & 536.5 & -37 \\
\hline $\mathrm{VBES}(\mathrm{mV}) @ \mathrm{IC}=100 \mathrm{~mA}, \mathrm{IB}=10 \mathrm{~mA}$ & 1112.5 & 1110.5 & 2 & 1160.5 & 1166 & -5.5 \\
\hline VBEON $(\mathrm{mV}) @ \mathrm{VCE}=5 \mathrm{~V}, \mathrm{IC}=5 \mathrm{~mA}$ & 716.5 & 716 & 0.5 & 731 & 732 & -1 \\
\hline $\mathrm{VBEON}(\mathrm{mV}) @ \mathrm{VCE}=5 \mathrm{~V}, \mathrm{IC}=20 \mathrm{~mA}$ & 768 & 769.5 & -1.5 & 789.5 & 788 & 1.5 \\
\hline$I C B D(n A) @ V C B=-50 V$ & 1.4 & 0.58 & 0.82 & 1.5 & 0.65 & 0.85 \\
\hline $\mathrm{ICBO}(\mathrm{nA}) @ \mathrm{VCB}=-70 \mathrm{~V}$ & 1.6 & 0.6 & 1 & 1.8 & 0.8 & 1 \\
\hline $\operatorname{ICES}(\mathrm{nA}) @ V C E=-50 \mathrm{~V}$ & 0.72 & 0.32 & 0.4 & 0.88 & 0.4 & 0.48 \\
\hline $\operatorname{ICER}(\mathrm{nA}) @ V C=-50 \mathrm{~V}, \mathrm{R}=1 \mathrm{~K}$ & 0.62 & 0.28 & 0.34 & 0.72 & 0.34 & 0.38 \\
\hline $\mathrm{IEBO}(\mathrm{nA})$ (1) VEB $=-5 \mathrm{~V}$ & 0.01 & 0.01 & 0 & 0.01 & 0.01 & 0 \\
\hline $\mathrm{VCES}(\mathrm{mV}) @ \mathrm{IC}=-10 \mathrm{~mA}, \mathrm{IB}=-500 \mu \mathrm{A}$ & 81 & 84.5 & -3.5 & 83.5 & 88 & -4.5 \\
\hline VBES $(\mathrm{mV}) @ \mathrm{IC}=-10 \mathrm{~mA}, \mathrm{IB}=-500 \mu \mathrm{A}$ & 761.5 & 762 & -0.5 & 766 & 768 & -2 \\
\hline VBEON $(m \mathrm{~V}) @ V C E=-5 \mathrm{~V}, \mathrm{IC}=-5 \mathrm{~mA}$ & 700.5 & 699 & 1.5 & 707 & 706 & 1 \\
\hline VBEON $(m V) @ V C E=-5 V, I C=-20 m A$ & 741.5 & 742 & -0.5 & 747.5 & 749 & -1.5 \\
\hline $1+\mathrm{HFE} @ \mathrm{VCB}=-5 \mathrm{~V}, \mathrm{IE}=-10 \mu \mathrm{A}$ & 212 & 240 & -28 & 227 & 261 & .34 \\
\hline HFE @ VCE $=-5 \mathrm{~V}, \mathrm{IC}=-500 \mu \mathrm{A}$ & 207 & 233 & -26 & 220 & 253 & -33 \\
\hline HFE@ VCE $=-5 \mathrm{~V}, I \mathrm{IC}=-10 \mathrm{~mA}$ & 193.8 & 217 & -23.3 & 206 & 237 & -31 \\
\hline HFE @ VCE $=-5 \mathrm{~V}, \mathrm{IC}=-20 \mathrm{~mA}$ & 181.8 & 204 & -22.2 & 193.2 & 221 & -27.8 \\
\hline
\end{tabular}




\begin{tabular}{|c|c|c|c|c|c|c|}
\hline TEST & AVE & AGE VALU & 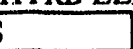 & STANI & ARD DEVIA & \\
\hline & Freon & DI Water & Delta & Freon & DI Water & Delta \\
\hline $\mathrm{IR}(\mathrm{nA}) @ \mathrm{VR}=55 \mathrm{~V}$ & 12 & 18 & -6 & 1.3 & 2 & -0.7 \\
\hline $\mathrm{VF}(\mathrm{mV}) @ \mathrm{IF}=1 \mathrm{~mA}$ & 626.01 & 622.71 & 3.3 & 2.85 & 3.16 & -0.31 \\
\hline $\mathrm{VF}(\mathrm{mV}) @ \mathrm{IF}=10 \mathrm{~mA}$ & 754.75 & 753.15 & 1.6 & 4.22 & 4.4 & -0.18 \\
\hline $\mathrm{ICBO}(\mathrm{nA}) @ \mathrm{VCB}=-50 \mathrm{~V}$ & 1.9 & 0.75 & 1.15 & 0.01 & 0.021 & -0.011 \\
\hline $\mathrm{ICBO}(\mathrm{nA}) @ \mathrm{VCB}=-70 \mathrm{~V}$ & 1.9 & 0.71 & 1.19 & 0.038 & 0.048 & -0.01 \\
\hline $\operatorname{ICES}(\mathrm{nA}) @ \mathrm{VCE}=-50 \mathrm{~V}$ & 1 & 0.43 & 0.57 & 0 & 0.022 & -0.022 \\
\hline ICER $(n A) @ V C=-50 V, R=1 K$ & 0.79 & 0.32 & 0.47 & 0.01 & 0.011 & 0.001 \\
\hline $\operatorname{IEBO}(\mathrm{nA}) @ \mathrm{VEB}=-5 \mathrm{~V}$ & 0.01 & 0.01 & 0 & 0 & 0 & 0 \\
\hline $\begin{array}{l}\text { VCES }(\mathrm{mV}) @ \mathrm{IC}=-10 \mathrm{~mA}, \mathrm{IB}=- \\
500 \mu \mathrm{A}\end{array}$ & 81.67 & 85.62 & -3.95 & 0.911 & 0.623 & 0.288 \\
\hline VBES $(\mathrm{mV}) @ \mathrm{IC}=-10 \mathrm{~mA}, \mathrm{IB}=-500 \mu \mathrm{A}$ & 764.71 & 763.23 & 1.48 & 0.914 & 0.907 & 0.007 \\
\hline $\mathrm{VBEON}(\mathrm{mV}) @ \mathrm{VCE}=-5 \mathrm{~V}, \mathrm{IC}=-5 \mathrm{~mA}$ & 677.74 & 700.01 & -22.27 & 3.66 & 1.09 & 2.57 \\
\hline $\mathrm{VBEON}(\mathrm{mV}) @ \mathrm{VCE}=-5 \mathrm{~V}, I \mathrm{C}=-20 \mathrm{~mA}$ & 707.89 & 742.83 & -34.94 & 9.4 & 1.01 & 8.39 \\
\hline $1+\mathrm{HFE} @ \mathrm{VCB}=-5 \mathrm{~V}, \mathrm{IE}=-10 \mu \mathrm{A}$ & 229.33 & 260.95 & .31 .62 & 5.0395 & 2.7245 & 2.315 \\
\hline $\mathrm{HFE} @ \mathrm{VCE}=-5 \mathrm{~V}, \mathrm{IC}=-500 \mu \mathrm{A}$ & 222.89 & 252.53 & -29.64 & 4.757 & 2.3793 & 2.3777 \\
\hline HFE @ VCE $=-5 \mathrm{~V}, \mathrm{IC}=-10 \mathrm{~mA}$ & 208.76 & 235.87 & -27.11 & 4.0837 & 2.2448 & 1.8389 \\
\hline $\mathrm{HFE} @ \mathrm{VCE}=-5 \mathrm{~V}, \mathrm{IC}=-20 \mathrm{~mA}$ & 369.74 & 220.84 & 148.9 & 21.398 & 2.0884 & 19.31 \\
\hline $\mathrm{ICBO}(\mathrm{nA}) @ \mathrm{VCB}=20 \mathrm{~V}$ & 3.6 & 3.6 & 0 & 0.41 & 0.27 & 0.14 \\
\hline $\operatorname{IEBO}(\mathrm{nA}) @ \mathrm{VEB}=4 \mathrm{~V}$ & 28 & 19 & 9 & 28 & 16 & 12 \\
\hline $\operatorname{ICER}(n A) @ V C E=13 V, R=1 \mathrm{~K}$ & 2.7 & 2.8 & -0.1 & 0.15 & 0.16 & -0.01 \\
\hline $\mathrm{HFE} @ \mathrm{VCE}=5 \mathrm{~V}, \mathrm{IC}=1 \mathrm{~mA}$ & 70.77 & 74.975 & -4.205 & 15.848 & 9.8259 & 6.0221 \\
\hline $\mathrm{HFE} @ \mathrm{VCE}=5 \mathrm{~V}, \mathrm{IC}=10 \mathrm{~mA}$ & 91.12 & 97.136 & -6.016 & 18.315 & 11.641 & 6.674 \\
\hline $\mathrm{HFE} @ \mathrm{VCE}=5 \mathrm{~V}, \mathrm{IC}=100 \mathrm{rnA}$ & 67.748 & 72.953 & -5.205 & 11.791 & 7.5656 & 4.2254 \\
\hline $\operatorname{VCES}(\mathrm{mV}) @ \mathrm{IC}=10 \mathrm{~mA}, I \mathrm{~B}=1 \mathrm{~mA}$ & 1.44 .47 & 143.52 & 0.95 & 4.06 & 3.95 & 0.11 \\
\hline $\operatorname{VBES}(\mathrm{mV}) @ I \mathrm{IC}=10 \mathrm{~mA}, I \mathrm{IB}=1 \mathrm{~mA}$ & 803.83 & 800.57 & 3.26 & 1.86 & 1.63 & 0.23 \\
\hline $\operatorname{VCES}(\mathrm{mV}) @ I C=100 \mathrm{~mA}, I \mathrm{~B}=10 \mathrm{~mA}$ & 470.77 & 472.31 & -1.54 & 26.49 & 20.95 & 5.54 \\
\hline $\operatorname{VBES}(\mathrm{mV}) @ I \mathrm{IC}=100 \mathrm{~mA}, \mathrm{IB}=10 \mathrm{~mA}$ & 1131.8 & $\Pi 137.8$ & -6 & 8.35 & 17.07 & -8.72 \\
\hline $\operatorname{VBEON}(\mathrm{mV}) @ \mathrm{VCE}=5 \mathrm{~V}, \mathrm{IC}=5 \mathrm{~mA}$ & 726.78 & 720.76 & 6.02 & 3.28 & 3.66 & -0.38 \\
\hline VBEON $(\mathrm{mV})$ @ VCE $=5 \mathrm{~V}, I \mathrm{IC}=10 \mathrm{~mA}$ & 782.28 & 775.9 & 6.38 & 5.09 & 4.5 & 0.59 \\
\hline $\mathrm{ICBO}(\mathrm{nA}) @ \mathrm{VCB}=-50 \mathrm{~V}$ & 1.5 & 0.56 & 0.94 & 0 & 0.018 & -0.018 \\
\hline $\mathrm{ICBO}(\mathrm{nA}) @ \mathrm{VCB}=-70 \mathrm{~V}$ & 1.7 & 0.63 & 1.07 & 0.045 & 0.046 & -0.001 \\
\hline $\mathrm{ICES}(\mathrm{nA}) @ \mathrm{VCE}=-50 \mathrm{~V}$ & 0.84 & 0.34 & 0.5 & 0.027 & 0.017 & 0.01 \\
\hline $\operatorname{ICER}(n A) @ V C=-50 V, R=1 K$ & 0.69 & 0.29 & 0.4 & 0.015 & 0.023 & -0.008 \\
\hline IEBO $(n A) @ V E B=-5 V$ & 0.01 & 0.01 & 0 & 0 & 0 & \\
\hline $\operatorname{VCES}(\mathrm{mV}) @ \mathrm{IC}=-10 \mathrm{~mA}, \mathrm{IB}=-500 \mu \mathrm{A}$ & 82.31 & 86.03 & -3.72 & 0.785 & 0.445 & 0.34 \\
\hline VBES $(\mathrm{mV}) @ \mathrm{IC}=-10 \mathrm{~mA}, \mathrm{IB}=-500 \mu \mathrm{A}$ & 764.45 & 763.39 & 1.06 & 0.859 & 1.32 & -0.461 \\
\hline $\mathrm{VBEON}(\mathrm{mV}) @ \mathrm{VCE}=-5 \mathrm{~V}, \mathrm{IC}=-5 \mathrm{~mA}$ & 704.87 & 700.43 & 4.44 & 1.17 & 1.35 & -0.18 \\
\hline VBEON (mV) @ VCE $=-5 \mathrm{~V}, \mathrm{IC}=-20 \mathrm{~mA}$ & 746.03 & 743.31 & 2.72 & 1.13 & 1.37 & -0.24 \\
\hline $1+\mathrm{HFE} @ \mathrm{VCB}=-5 \mathrm{~V}, \mathrm{IE}=-10 \mu \mathrm{A}$ & 220.3 & 249.87 & -29.57 & 4.9929 & 3.8637 & 1.1292 \\
\hline $\mathrm{HFE} @ \mathrm{VCE}=-5 \mathrm{~V}, \mathrm{IC}=-500 \mu \mathrm{A}$ & 213.67 & 241.91 & .28 .24 & 4.8252 & 3.4441 & 1.3811 \\
\hline HFE @ VCE $=-5 \mathrm{~V}, \mathrm{IC}=-10 \mathrm{~mA}$ & 200.23 & 225.65 & -25.42 & 4.4751 & 3.2954 & 1.1797 \\
\hline $\mathrm{HFE} @ \mathrm{VCE}=-5 \mathrm{~V}, \mathrm{IC}=-20 \mathrm{~mA}$ & 187.66 & 211.35 & -23.69 & 3.9734 & 2.8689 & 1.1045 \\
\hline $\mathrm{IR}(\mathrm{nA}) @ \mathrm{VR}=55 \mathrm{~V}$ & 12 & 17 & -5 & 1.5 & 1.8 & -0.3 \\
\hline $\mathrm{VF}(\mathrm{mV}) @ \mathrm{IF}=1 \mathrm{~mA}$ & 627.56 & 625.17 & 2.39 & 2.17 & 2.99 & -0.82 \\
\hline $\mathrm{VF}(\mathrm{mV}) @ \mathrm{IF}=10 \mathrm{~mA}$ & 757.16 & 756.05 & 1.11 & 3.32 & 4.05 & -0.73 \\
\hline $\mathrm{ICBO}(\mathrm{nA}) @ \mathrm{VCB}=-50 \mathrm{~V}$ & 1.9 & 0.72 & 1.18 & 0.0091 & 0.02 & -0.011 \\
\hline $\mathrm{ICBO}(\mathrm{nA}) @ \mathrm{VCB}=-70 \mathrm{~V}$ & 1.9 & 0.7 & 1.2 & 0.035 & 0.05 & -0.015 \\
\hline $\mathrm{ICES}(\mathrm{nA}) @ \mathrm{VCE}=-50 \mathrm{~V}$ & 1 & 0.44 & 0.56 & 0 & 0.024 & -0.024 \\
\hline $\operatorname{ICER}(\mathrm{nA}) @ \mathrm{VC}=-50 \mathrm{~V}, \mathrm{R}=1 \mathrm{~K}$ & 0.78 & 0.33 & 0.45 & 0.01 & 0.014 & -0.004 \\
\hline IEBO $(n A) @$ VEB $=-5 V$ & 0.01 & 0.01 & 0 & 0 & 0 & \\
\hline $\begin{array}{l}\text { VCES (mV)@ IC }=-10 \mathrm{~mA}, \mathrm{IB}=- \\
500 \mu \mathrm{A}\end{array}$ & 81.75 & 85.66 & -3.91 & 0.805 & 0.601 & 0.204 \\
\hline $\operatorname{VBES}(\mathrm{mV}) @ \mathrm{IC}=10 \mathrm{~mA}, \mathrm{ID}--500, \mathrm{~A}$ & 764.83 & 763.77 & 1.06 & 0.962 & 1.24 & .0 .278 \\
\hline
\end{tabular}




\begin{tabular}{|c|c|c|c|c|c|c|}
\hline \multicolumn{7}{|c|}{$\begin{array}{l}\text { TABLE A28: AVERAGE AND STANDARD DEVIATION OF POST HTRB ELECTRICAL TEST DATA FOR M-50 } \\
\text { (CONTINUED) }\end{array}$} \\
\hline \multirow[t]{2}{*}{ TEST } & \multicolumn{3}{|c|}{ AVERAGE VALUES } & \multicolumn{3}{|c|}{ STANDARD DEVIATION } \\
\hline & Freon & DI Water & Delta & Freon & DI Water & Deita \\
\hline VBEON (mV) @ VCE $=-5 \mathrm{~V}, \mathrm{IC}=-5 \mathrm{~mA}$ & 704.72 & 700.59 & 4.13 & 1.11 & 1.5 & -0.39 \\
\hline VBEON $(m \mathrm{~m}) @ V C E=-5 V, I C=-20 \mathrm{~mA}$ & 741.77 & 743.37 & -1.6 & 1.11 & 1.44 & -0.33 \\
\hline $1+\mathrm{HFE} @ \mathrm{VCB}=-5 \mathrm{~V}, \mathrm{IE}=-10 \mu \mathrm{A}$ & 231.28 & 259.31 & -28.03 & 4.7679 & 3.7311 & 1.0368 \\
\hline HFE @ VCE $=-5 \mathrm{~V}, \mathrm{IC}=-500 \mu \mathrm{A}$ & 224.7 & 251.18 & -26.48 & 4.3595 & 3.1334 & 1.2261 \\
\hline $\mathrm{HFE} @ \mathrm{VCE}=-5 \mathrm{~V}, I \mathrm{C}=-10 \mathrm{~mA}$ & 210.22 & 234.38 & -24.16 & 3.8494 & 3.052 & 0.7974 \\
\hline HFE @ VCE $=-5 \mathrm{~V}, \mathrm{IC}=-20 \mathrm{~mA}$ & 196.92 & 219.53 & -22.61 & 3.5799 & 2.8209 & 0.759 \\
\hline $\mathrm{ICBO}(\mathrm{nA}) @ \mathrm{VCB}=20 \mathrm{~V}$ & 3.3 & 3.7 & .0 .4 & 0.4 & 0.65 & -0.25 \\
\hline $\mathrm{IEBO}(\mathrm{nA}) @ \mathrm{VEB}=4 \mathrm{~V}$ & 34 & 26 & 8 & 43 & 26 & 17 \\
\hline $\operatorname{ICER}(\mathrm{nA}) @ \mathrm{VCE}=13 \mathrm{~V}, \mathrm{R}=1 \mathrm{~K}$ & 2.5 & 2.8 & -0.3 & 0.25 & 0.18 & 0.07 \\
\hline $\mathrm{HFE} @ \mathrm{VCE}=5 \mathrm{~V}, \mathrm{IC}=1 \mathrm{~mA}$ & 91.344 & 65.835 & 25.509 & 22.318 & 9.643 & 12.675 \\
\hline $\mathrm{HFE} @ \mathrm{VCE}=5 \mathrm{~V}, \mathrm{IC}=10 \mathrm{~mA}$ & 113.42 & 85.502 & 27.918 & 24.751 & 11.551 & 13.2 \\
\hline $\mathrm{HFE} @ \mathrm{VCE}=5 \mathrm{~V}, \mathrm{IC}=100 \mathrm{~mA}$ & 81.298 & 65.007 & 16.291 & 15.237 & 7.4442 & 7.7928 \\
\hline VCES $(\mathrm{mV}) @ \mathrm{IC}=10 \mathrm{~mA}, \mathrm{IB}=1 \mathrm{~mA}$ & 138.69 & 148.28 & -9.59 & 4.33 & 3.97 & 0.36 \\
\hline $\operatorname{VBES}(\mathrm{mV}) @ \mathrm{IC}=10 \mathrm{~mA}, \mathrm{IB}=1 \mathrm{~mA}$ & 802.67 & 800.92 & 1.75 & 2.29 & 1.6 & 0.69 \\
\hline VCES(mV)@IC $=100 \mathrm{~mA}, I B=10 \mathrm{~mA}$ & 438.79 & 496.16 & -57.37 & 24.14 & 19.96 & 4.18 \\
\hline $\operatorname{VBES}(\mathrm{mV}) @ \mathrm{IC}=100 \mathrm{~mA}, \mathrm{IB}=10 \mathrm{~mA}$ & 1131.9 & 1122.4 & 9.5 & 11.53 & 11.39 & 0.14 \\
\hline $\mathrm{VBEON}(\mathrm{mV}) @ \mathrm{VCE}=5 \mathrm{~V}, \mathrm{IC}=5 \mathrm{~mA}$ & 723.79 & 725.06 & .1 .27 & $4.9 !$ & 3.72 & 0.29 \\
\hline VBEON $(\mathrm{mV}) @ \mathrm{VCE}=5 \mathrm{~V}, \quad \mathrm{IC}=20 \mathrm{~mA}$ & 777.7 & 780.94 & -3.24 & 6.51 & 4.46 & 1.85 \\
\hline$I C B O(n A) @ V C B=-50 V$ & 1.5 & 0.62 & 0.88 & 0.022 & 0.018 & 0.004 \\
\hline $\mathrm{ICBO}(\mathrm{nA}) @ \mathrm{VCB}=-70 \mathrm{~V}$ & 1.7 & 0.72 & 0.98 & 0.054 & 0.059 & -0.005 \\
\hline $\operatorname{ICES}(\mathrm{nA}) @ \mathrm{VCE}=-50 \mathrm{~V}$ & 0.81 & 0.38 & 0.43 & 0.048 & 0.023 & 0.025 \\
\hline $\operatorname{ICER}(\mathrm{nA}) @ \mathrm{VC}=.50 \mathrm{~V}, \mathrm{R}=1 \mathrm{~K}$ & 0.66 & 0.31 & 0.35 & 0.02 & 0.012 & 0.008 \\
\hline IEBO (nA)@ VEB =-5V & 0.01 & 0.01 & 0 & 0 & 0 & 0 \\
\hline $\mathrm{VCES}(\mathrm{mV}) @ \mathrm{IC}=-10 \mathrm{~mA}, \mathrm{IB}=-500 \mu \mathrm{A}$ & 82.14 & 86.54 & -4.4 & 0.736 & 0.679 & 0.057 \\
\hline $\mathrm{VBES}(\mathrm{mV}) @ \mathrm{IC}=-10 \mathrm{~mA}, \mathrm{IB}=-500 \mu \mathrm{A}$ & 764.57 & 763.88 & 0.69 & 0.939 & 1.32 & -0.381 \\
\hline VBEON $(m V) @ V C E=-5 V, I C=-5 \mathrm{~mA}$ & 704.61 & 700.75 & 3.86 & 1.19 & 1.58 & .0 .39 \\
\hline VBEON $(\mathrm{mV}) @ \mathrm{VCE}=-5 \mathrm{~V}, \mathrm{IC}=-20 \mathrm{~mA}$ & 745.54 & 743.63 & 1.91 & 1.26 & 1.51 & -0.25 \\
\hline $1+\mathrm{HFE} @ \mathrm{VCB}=-5 \mathrm{~V}, \mathrm{IE}=-10 \mu \mathrm{A}$ & 223.09 & 249.67 & -26.58 & 3.8767 & 3.4105 & 0.4662 \\
\hline HFE @ VCE $=-5 \mathrm{~V}, \mathrm{IC}=-500 \mu \mathrm{A}$ & 216.37 & 242.02 & -25.65 & 3.5358 & 3.1298 & 0.406 \\
\hline HFE @VCE $=-5 \mathrm{~V}, \mathrm{IC}=-10 \mathrm{~mA}$ & 202.68 & 115.82 & 86.86 & 3.3104 & 2.9001 & 0.4103 \\
\hline HFE@VCE $=-5 \mathrm{~V}, \mathrm{IC}=-20 \mathrm{~mA}$ & 189.65 & 211.33 & -21.68 & 2.8728 & 2.539 & 0.3338 \\
\hline
\end{tabular}




\begin{tabular}{|c|c|c|c|c|c|c|}
\hline \multirow[t]{2}{*}{ TEST } & \multicolumn{3}{|c|}{ MINIMUM VALUES } & \multicolumn{3}{|c|}{ MAXIMUM VALUES } \\
\hline & Freon & DI Water & Delta & Freon & DI Water & Delta \\
\hline $\mathrm{IR}(\mathrm{nA}) @ \mathrm{VR}=55 \mathrm{~V}$ & 12 & 7.7 & 4.3 & 12 & 16 & -4 \\
\hline$\overline{V F}(\mathrm{mV}) @ I F=I m A$ & 620 & 619 & 1 & 630 & 633.5 & -3.5 \\
\hline $\mathrm{VF}(\mathrm{mV}) @ \mathrm{II}=10 \mathrm{~mA}$ & 748 & 743.5 & 4.5 & 761.5 & 764 & .2 .5 \\
\hline $\mathrm{ICBO}(\mathrm{nA}) @ \mathrm{VCB}=-50 \mathrm{~V}$ & 1.6 & 0.8 & 0.8 & 1.7 & 0.81 & 0.89 \\
\hline $\mathrm{ICBO}(\mathrm{nA}) @ \mathrm{VCB}=-70 \mathrm{~V}$ & 1.5 & 0.7 & 0.8 & 1.8 & 0.8 & 1 \\
\hline $\operatorname{ICES}(n A) @ V C E=-50 \mathrm{~V}$ & 0.84 & 0.48 & 0.36 & 0.94 & 0.48 & 0.46 \\
\hline $\operatorname{ICER}(\mathrm{nA}) @ \mathrm{VC}=-50 \mathrm{~V}, \mathrm{R}=1 \mathrm{~K}$ & 0.64 & 0.38 & 0.26 & 0.77 & 0.4 & 0.37 \\
\hline $\operatorname{IEBO}(\mathrm{nA}) @ \mathrm{VEB}=-5 \mathrm{~V}$ & 0.005 & 0.035 & -0.03 & 0.005 & 0.055 & -0.05 \\
\hline $\begin{array}{l}\mathrm{VCES}(\mathrm{mV}) @ \mathrm{IC}=-10 \mathrm{~mA}, \mathrm{IB}=- \\
500 \mu \mathrm{A}\end{array}$ & 80.5 & 83.5 & -3 & 83 & 85.5 & -2.5 \\
\hline $\mathrm{VBES}(\mathrm{mV}) @ \mathrm{IC}=-10 \mathrm{~mA}, \mathrm{IB}=-500 \mu \mathrm{A}$ & 764.5 & 767 & -2.5 & 766 & 768.5 & -2.5 \\
\hline $\mathrm{VBEON}(\mathrm{mV}) @ \mathrm{VCE}=-5 \mathrm{~V}, \mathrm{IC}=-5 \mathrm{~mA}$ & 704 & 704 & 0 & 706 & 707 & -1 \\
\hline VBEON $(m V) @ V C E=-5 V, I C=-20 m A$ & 727.5 & 747 & -19.5 & 740 & 749 & -9 \\
\hline $1+\mathrm{HFE} @ \mathrm{VCB}=-5 \mathrm{~V}, \mathrm{IE}=-10 \mu \mathrm{A}$ & 219 & 246 & -27 & 235 & 258 & -23 \\
\hline $\mathrm{HFE} @ \mathrm{VCE}=-5 \mathrm{~V}, \mathrm{IC}=-500 \mu \mathrm{A}$ & 214 & 239 & .25 & 227 & 251 & -24 \\
\hline HFE @ VCE $=-5 V, I C=-10 \mathrm{mi}$ & 201 & 223 & -22 & 212 & 234 & .22 \\
\hline $\mathrm{HFE} @ \mathrm{VCE}=-5 \mathrm{~V}, \mathrm{IC}=-20 \mathrm{~mA}$ & 208 & 209 & -1 & 245 & 219 & 26 \\
\hline $\mathrm{ICBO}(\mathrm{nA}) @ \mathrm{VCB}=20 \mathrm{~V}$ & 3 & 2.2 & 0.8 & 4.9 & 3 & 1.9 \\
\hline IEBO (nA)@VEB=4V & 13 & 12 & 1 & 66 & 150 & -84 \\
\hline $\mathrm{ICER}(\mathrm{nA}) @ \mathrm{VCE}=13 \mathrm{~V}, \mathrm{R}=1 \mathrm{~K}$ & 2.6 & 1.8 & 0.8 & 2.9 & 2.1 & 0.8 \\
\hline $\mathrm{HFE} @ \mathrm{VCE}=5 \mathrm{~V}, \mathrm{IC}=1 \mathrm{~mA}$ & 50.9 & 56.4 & -5.5 & 104.2 & 84.9 & 19.3 \\
\hline HFE @ VCE $=5 \mathrm{~V}, \mathrm{IC}=10 \mathrm{~mA}$ & 69.2 & 73.5 & -4.3 & 129.2 & 108.1 & 21.1 \\
\hline $\mathrm{HHE} @ \mathrm{VCE}=5 \mathrm{~V}, \mathrm{IC}=100 \mathrm{~mA}$ & 53.6 & 57.1 & -3.5 & 93.3 & 79.4 & 13.9 \\
\hline VCES $(m V) @ I C=10 m A, I B=1 m A$ & 135.5 & 139.5 & -4 & 148.5 & 152 & -3.5 \\
\hline $\operatorname{VBES}(\mathrm{mV}) @ \mathrm{IC}=10 \mathrm{~mA}, \mathrm{IB}=1 \mathrm{~mA}$ & 800.5 & 803.5 & -3 & 806 & 809.5 & -3.5 \\
\hline $\operatorname{VCES}(\mathrm{mV}) @ \mathrm{IC}=100 \mathrm{~mA}, \mathrm{IB}=10 \mathrm{~mA}$ & 419.5 & 448 & -28.5 & 499 & 520 & -21 \\
\hline $\mathrm{VBES}(\mathrm{mV}) @ \mathrm{IC}=100 \mathrm{~mA}, \mathrm{IB}=10 \mathrm{~mA}$ & 1119 & 1115 & 4 & 1146 & 1181.5 & -35.5 \\
\hline VBEON $(\mathrm{mV}) @ \mathrm{VCE}=5 \mathrm{~V}, \mathrm{IC}=5 \mathrm{~mA}$ & 720 & 723.5 & -3.5 & 731 & 732.5 & -1.5 \\
\hline VBEON $(\mathrm{mV}) @ \mathrm{VCE}=5 \mathrm{~V}, I \mathrm{C}=10 \mathrm{~mA}$ & 772 & 777 & -5 & 789.5 & 790.5 & -1 \\
\hline $\mathrm{ICBO}(\mathrm{nA}) @ \mathrm{VCB}=-50 \mathrm{~V}$ & 1.5 & 0.6 & 0.9 & 1.6 & 0.63 & 0.97 \\
\hline $\mathrm{ICBO}(\mathrm{nA}) @ \mathrm{VCB}=.70 \mathrm{~V}$ & 1.5 & 0.6 & 0.9 & 1.9 & 0.8 & 1.1 \\
\hline ICES (nA)@VCE $=-50 \mathrm{~V}$ & 0.8 & 0.38 & 0.42 & 0.9 & 0.4 & 0.5 \\
\hline $\operatorname{ICER}(\mathrm{nA}) @ \mathrm{VC}=-50 \mathrm{~V}, \mathrm{R}=1 \mathrm{~K}$ & 0.64 & 0.32 & 0.32 & 0.76 & 0.34 & 0.42 \\
\hline IEBO (nA) \& VEB $=-5 \mathrm{~V}$ & .0 .01 & 0.01 & 0 & 0.005 & 0.03 & -0.025 \\
\hline $\mathrm{VCES}(\mathrm{mV}) @ \mathrm{IC}=-10 \mathrm{~mA}, \mathrm{IB}=-500 \mu \mathrm{A}$ & 81.5 & 83.5 & .2 & 83.5 & 86 & -2.5 \\
\hline $\operatorname{VBES}(m V) @ I C=-10 m A, I B=-500 \mu A$ & 764 & 766 & -2 & 765.5 & 768 & -2.5 \\
\hline $\mathrm{VBEON}(\mathrm{mV}) @ \mathrm{VCE}=-5 \mathrm{~V}, \mathrm{IC}=-5 \mathrm{~mA}$ & 704 & 704 & 0 & 705.5 & 706 & -0.5 \\
\hline VBEON $(\mathrm{mV}) @ \mathrm{VCE}=-5 \mathrm{~V}, \mathrm{IC}=-20 \mathrm{~mA}$ & 747.5 & 747 & 0.5 & 749 & 748.5 & 0.5 \\
\hline $1+\mathrm{HFE} @ \mathrm{VCB}=-5 \mathrm{~V}, \mathrm{IE}=-10 \mu \mathrm{A}$ & 210 & 238 & -28 & 223 & 256 & -33 \\
\hline $\mathrm{HFE} @ \mathrm{VCE}=-5 \mathrm{~V}, \mathrm{IC}=-500 \mu \mathrm{A}$ & 204 & 230 & -26 & 216 & 248 & -32 \\
\hline HFE@ Q VCE $=-5 \mathrm{~V}, I \mathrm{C}=-10 \mathrm{~mA}$ & 191.2 & 215 & -23.8 & 202 & 231 & -29 \\
\hline $\mathrm{HFE} @ \mathrm{VCE}=-5 \mathrm{~V}, \mathrm{IC}=-20 \mathrm{~mA}$ & 179.5 & 202 & -22.5 & 189.7 & 216 & -26.3 \\
\hline $\mathrm{IR}(\mathrm{nA}) @ \mathrm{VR}=55 \mathrm{~V}$ & 10 & 7.9 & 2.1 & 14 & 15 & -1 \\
\hline $\mathrm{VF}(\mathrm{mV}) @ \mathrm{IF}=1 \mathrm{~mA}$ & 624 & 626 & -2 & 631 & 634 & -3 \\
\hline $\mathrm{VF}(\mathrm{mV}) @ \mathrm{IF}=10 \mathrm{~mA}$ & 750 & 752 & -2 & 761.5 & 764 & -2.5 \\
\hline $\mathrm{ICBO}(\mathrm{nA}) @ \mathrm{VCB}=-50 \mathrm{~V}$ & 1.6 & 0.77 & 0.83 & 1.6 & 0.79 & 0.81 \\
\hline $\mathrm{ICBO}(\mathrm{nA}) @ \mathrm{VCB}=-70 \mathrm{~V}$ & 1.5 & 0.65 & 0.85 & 1.7 & 0.8 & 0.9 \\
\hline ICES $(n A) @ V C E=-50 \mathrm{~V}$ & 0.87 & 0.48 & 0.39 & 0.92 & 0.49 & 0.43 \\
\hline $\operatorname{ICER}(\mathrm{nA}) @ \mathrm{VC}=-50 \mathrm{~V}, \mathrm{R}=1 \mathrm{~K}$ & 0.66 & 0.38 & 0.28 & 0.71 & 0.4 & 0.31 \\
\hline IEBO (nA)@ VEB $=-5 \mathrm{~V}$ & 0.002 & 0.015 & -0.01 & 0.005 & 0.055 & -0.05 \\
\hline $\begin{array}{l}\text { VCES }(m V) @ I C=-10 m A, I B=- \\
500 \mu A\end{array}$ & 80.5 & 83.5 & -3 & 83 & 85.5 & -2.5 \\
\hline
\end{tabular}




\begin{tabular}{|c|c|c|c|c|c|c|}
\hline \multirow[t]{2}{*}{ TEST } & \multicolumn{3}{|c|}{ MINIMUM VALUES } & \multicolumn{3}{|c|}{ MAXIMUM VALUES } \\
\hline & Freon & DI Water & Delta & Freon & DI Water & Delta \\
\hline VBES (mV)@IC $=-10 \mathrm{~mA}, I B=-500 \mu \mathrm{A}$ & 764.5 & 767 & -2.5 & 765.5 & 768.5 & -3 \\
\hline VBEON $(\mathrm{mV}) @ \mathrm{VCE}=-5 \mathrm{~V}, \mathrm{IC}=-5 \mathrm{~mA}$ & 704.5 & 704 & 0.5 & 705.5 & 706 & -0.5 \\
\hline VBEON $(m V) @ V C . E=-5 V, I C=-20 m A$ & 744 & 746.5 & -2.5 & 746 & 749 & -3 \\
\hline $1+\mathrm{HFE} @ \mathrm{VCB}=-5 \mathrm{~V}, \mathrm{IE}=-10 \mu \mathrm{A}$ & 221 & 251 & -30 & 236 & 257 & .21 \\
\hline $\mathrm{HFE} @ \mathrm{VCE}=-5 \mathrm{~V}, \mathrm{IC}=-500 \mu \mathrm{A}$ & 215 & 243 & -28 & 230 & 249 & .19 \\
\hline $\mathrm{HFE} @ \mathrm{VCE}=-5 \mathrm{~V}, \mathrm{IC}=-10 \mathrm{~mA}$ & 202 & 227 & -25 & 215 & 232 & .17 \\
\hline $\mathrm{HFE} @ \mathrm{VCE}=-5 \mathrm{~V}, \mathrm{IC}=-20 \mathrm{~mA}$ & 189 & 212 & -23 & 201 & 217 & -16 \\
\hline $\mathrm{ICBO}(\mathrm{nA}) @ \mathrm{VCB}=20 \mathrm{~V}$ & 2.7 & 2.3 & 0.4 & 4,1 & 3.2 & 0.9 \\
\hline $\mathrm{IEBO}(\mathrm{nA}) @ \mathrm{VEB}=4 \mathrm{~V}$ & 12 & 12 & 0 & 101 & 49 & 52 \\
\hline$I C E R(n A) @ V C E=13 V, R=1 K$ & 2.1 & 1.8 & 0.3 & 3 & 2.1 & 0.9 \\
\hline $\mathrm{HFE} @ \mathrm{VCE}=5 \mathrm{~V}, \mathrm{IC}=1 \mathrm{~mA}$ & 53.7 & 56.2 & -2.5 & 125.6 & 92.3 & 33.3 \\
\hline $\mathrm{HFE} @ \mathrm{VCE}=5 \mathrm{~V}, \mathrm{IC}=10 \mathrm{~mA}$ & 72.5 & 74.1 & -1.6 & 149.3 & 116.7 & 32.6 \\
\hline $\mathrm{HFE} @ \mathrm{VCE}=5 \mathrm{~V}, \mathrm{IC}=100 \mathrm{~mA}$ & 56.2 & 57.3 & -1.1 & 102.7 & 84.5 & 18.2 \\
\hline $\mathrm{VCES}(\mathrm{mV}) @ \mathrm{IC}=10 \mathrm{~mA}, \mathrm{IB}=1 \mathrm{~mA}$ & 132.5 & 137.5 & -5 & 148 & 152 & 4 \\
\hline $\mathrm{VBES}(\mathrm{mV}) @ \mathrm{IC}=10 \mathrm{~mA}, \mathrm{IB}=1 \mathrm{~mA}$ & 799.5 & 804 & -4.5 & 806 & 807.5 & -1.5 \\
\hline $\mathrm{VCES}(\mathrm{mV}) @ \mathrm{IC}=100 \mathrm{~mA}, I B=10 \mathrm{~mA}$ & 412.5 & 437.5 & -25 & 495.5 & 535 & -39.5 \\
\hline VBES $(\mathrm{mV}) @ I C=100 \mathrm{~mA}, I B=10 \mathrm{~mA}$ & 1112.5 & 1114 & -1.5 & 1154.5 & 1168 & -13.5 \\
\hline $\operatorname{VBEON}(\mathrm{mV}) @ \mathrm{VCE}=5 \mathrm{~V}, \mathrm{IC}=5 \mathrm{~mA}$ & 718 & 723.5 & -5.5 & 729.5 & 733.5 & -4 \\
\hline $\mathrm{VBEON}(\mathrm{mV}) @ \mathrm{VCE}=5 \mathrm{~V}, \mathrm{IC}=20 \mathrm{~mA}$ & 769 & 776 & -7 & 787 & 789.5 & -2.5 \\
\hline $\mathrm{ICBO}(\mathrm{nA}) @ \mathrm{VCB}=-50 \mathrm{~V}$ & 1.3 & 0.66 & 0.64 & 1.3 & 0.68 & 0.62 \\
\hline $\mathrm{ICBO}(\mathrm{nA}) @ \mathrm{VCB}=-70 \mathrm{~V}$ & 1.4 & 0.7 & 0,7 & 1.6 & 0.8 & 0.8 \\
\hline $\operatorname{ICES}(\mathrm{nA}) @ \mathrm{VCE}=-50 \mathrm{~V}$ & 0.68 & 0.41 & 0.27 & 0.82 & 0.46 & 0.36 \\
\hline $\operatorname{ICER}(\mathrm{nA}) @ V C=-50 \mathrm{~V}, \mathrm{R}=1 \mathrm{~K}$ & 0.64 & 0.36 & 0.28 & 0.68 & 0.38 & 0.3 \\
\hline IEBO $(\mathrm{nA}) @ \mathrm{VEB}=-5 \mathrm{~V}$ & 0.01 & 0.01 & 0 & 0.01 & 0.01 & 0 \\
\hline $\mathrm{VCES}(\mathrm{mV}) @ \mathrm{IC}=-10 \mathrm{~mA}, \mathrm{IB}=-500 \mu \mathrm{A}$ & 81.5 & 83.5 & -2 & 83.5 & 87 & -3.5 \\
\hline $\operatorname{VBES}(\mathrm{mV}) @ \mathrm{IC}=-10 \mathrm{~mA}, \mathrm{IB}=-500 \mu \mathrm{A}$ & 764 & 767 & -3 & 765.5 & 768.5 & -3 \\
\hline VBEON (mV) @ VCE $=-5 \mathrm{~V}, 1 \mathrm{C}=-5 \mathrm{~mA}$ & 704 & 704 & 0 & 705.5 & 707 & -1.5 \\
\hline VBEON $(\mathrm{mV}) @ \mathrm{VCE}=-5 \mathrm{~V}, \mathrm{IC}=-20 \mathrm{~mA}$ & 747 & 747 & 0 & 748.5 & 749.5 & -1 \\
\hline $1+\mathrm{HFE} @ \mathrm{VCB}=-5 \mathrm{~V}, \mathrm{IE}=-10 \mu \mathrm{A}$ & 213 & 239 & .26 & 224 & 251 & -27 \\
\hline $\mathrm{HFE} @ \mathrm{VCE}=-5 \mathrm{~V}, \mathrm{IC}=-500 \mu \mathrm{A}$ & 207 & 232 & -25 & 217 & 243 & -26 \\
\hline HFE @ VCE $=-5 \mathrm{~V}, \mathrm{IC}=-10 \mathrm{~mA}$ & 193.8 & 216 & -22.2 & 204 & 227 & -23 \\
\hline HFE @ VCE $=-5 \mathrm{~V}, \mathrm{IC}=-20 \mathrm{~mA}$ & 182 & 203 & -21 & 190.2 & 213 & -22.8 \\
\hline
\end{tabular}




\begin{tabular}{|c|c|c|c|c|c|c|}
\hline \multicolumn{7}{|c|}{$\begin{array}{l}\text { TABLE A30: AVERAGE AND STANDARD DEVIATION OF POST EXTENDED HTRB ELECTRICAL TEST } \\
\text { FOR M-50 }\end{array}$} \\
\hline \multirow[t]{2}{*}{ TEST } & \multicolumn{3}{|c|}{ AVERAGE VALUES } & \multicolumn{3}{|c|}{ STANDARD DEVIATION } \\
\hline & Freon & DI Water & Delta & Freon & DI Water & Delta \\
\hline $\mathrm{IR}(\mathrm{nA}) @ \mathrm{VR}=55 \mathrm{~V}$ & 13 & 14 & -1 & 1.1 & 1.9 & -0.8 \\
\hline $\mathrm{VF}(\mathrm{mV}) @ \mathrm{IF}=\operatorname{ImA}$ & 626.19 & 62.9 .61 & -3.42 & 2.64 & 3.27 & -0.63 \\
\hline $\mathrm{VF}(\mathrm{mV}) @ \mathrm{IF}=10 \mathrm{~mA}$ & 754.81 & 759.31 & -4.5 & 3.91 & 4.53 & 0.62 \\
\hline $\mathrm{ICBO}(\mathrm{nA}) @ \mathrm{VCB}=-50 \mathrm{~V}$ & 1.7 & 0.8 & 0.9 & 0.023 & 0.0029 & 0.0201 \\
\hline $\mathrm{ICBO}(\mathrm{nA}) @ \mathrm{VCB}=-70 \mathrm{~V}$ & 1.6 & 0.78 & 0.82 & 0.1 & 0.031 & 0.069 \\
\hline $\operatorname{ICES}(\mathrm{nA}) @ V C E=-50 \mathrm{~V}$ & 0.88 & 0.48 & 0.4 & 0.03 & 0 & 0.03 \\
\hline $\operatorname{ICER}(\mathrm{nA}) @ \mathrm{VC}=-50 \mathrm{~V}, \mathrm{R}=1 \mathrm{~K}$ & 0.68 & 0.39 & 0.29 & 0.039 & 0.0078 & 0.0312 \\
\hline $\mathrm{IEBO}(\mathrm{nA}) @ \mathrm{VEB}=-5 \mathrm{~V}$ & 0.005 & 0.039 & -0.034 & 0 & 0.0059 & -0.0059 \\
\hline $\begin{array}{l}\text { VCES }(\mathrm{mV}) @ \mathrm{IC}=-10 \mathrm{~mA}, \mathrm{IB}= \\
500 \mu \mathrm{A}\end{array}$ & 81.59 & 84.19 & -2.6 & 0.821 & 0.489 & 0.332 \\
\hline $\operatorname{VBES}(\mathrm{mV}) @ \mathrm{IC}=-10 \mathrm{~mA}, \overline{\mathrm{IB}}=-500 \mu \mathrm{A}$ & 765 & 767.67 & -2.67 & 0.516 & 0.485 & 0.031 \\
\hline $\mathrm{VBEON}(\mathrm{mV}) @ \mathrm{VCE}=-5 \mathrm{~V}, \mathrm{IC}=-5 \mathrm{nA}$ & 704.97 & 704.94 & 0.03 & 0.591 & 0.856 & -0.265 \\
\hline VBEON $(\mathrm{mV}) @ \mathrm{VCE}=-5 \mathrm{~V}, \mathrm{IC}=-20 \mathrm{~mA}$ & 734.72 & 747.69 & $-12,97$ & 4.72 & 0.667 & 4.053 \\
\hline $1+\mathrm{HFE} @ \mathrm{VCB}=.5 \mathrm{~V}, \mathrm{IE}=-10 \mu \mathrm{A}$ & 226.94 & 255.28 & -28.34 & 5.4708 & 2.6746 & 2.7962 \\
\hline $\mathrm{HFE} @ \mathrm{VCE}=-5 \mathrm{~V}, \mathrm{IC}=-500 \mu \mathrm{A}$ & 220.63 & 247.22 & -26.59 & 4.7592 & 2.6247 & 2.1345 \\
\hline HFE@VCE $=-5 \mathrm{~V}, I \mathrm{IC}=-10 \mathrm{~mA}$ & 206.63 & 230.61 & .23 .98 & 4.0476 & 2.3798 & 1.6678 \\
\hline HFE @ VCE $=-5 \mathrm{~V}, \mathrm{IC}=-20 \mathrm{~mA}$ & 224.69 & 216.06 & 8.63 & 11.649 & 2.2089 & 9.4401 \\
\hline $\mathrm{ICBO}(\mathrm{nA}) @ \mathrm{VCB}=20 \mathrm{~V}$ & 3.6 & 2.5 & 1.1 & 0.48 & 0.18 & 0.3 \\
\hline $\mathrm{IEBO}(\mathrm{nA}) @ \mathrm{VEB}=4 \mathrm{~V}$ & 25 & 24 & 1 & 16 & 32 & -16 \\
\hline $\operatorname{ICER}(\mathrm{n} A) @ \mathrm{VCE}=13 \mathrm{~V}, \mathrm{R}=1 \mathrm{~K}$ & 2.7 & 2 & 0.7 & 0.081 & 0.0081 & 0.0729 \\
\hline $\mathrm{HFE} @ \mathrm{VCE}=5 \mathrm{~V}, \mathrm{IC}=1 \mathrm{~mA}$ & 74.425 & 69.428 & 4,997 & 18.011 & 9.046 & 8.965 \\
\hline $\mathrm{HFE} \otimes \mathrm{VCE}=5 \mathrm{~V}, \mathrm{IC}=10 \mathrm{~mA}$ & 95.6 & 90.633 & 4.967 & 21.133 & 11.009 & 10.124 \\
\hline $\mathrm{HFE} @ \mathrm{VCE}=5 \mathrm{~V}, \mathrm{IC}=100 \mathrm{~mA}$ & 70.65 & 68.839 & 1.811 & 13.693 & 7.3454 & 6.3476 \\
\hline $\mathrm{VCES}(\mathrm{mV}) @ \mathrm{IC}=10 \mathrm{~mA}, \mathrm{IB}=1 \mathrm{~mA}$ & $10,3.31$ & 144.17 & -0.86 & 4.99 & 4.35 & 0.64 \\
\hline VBES $(\mathrm{mV}) @ \mathrm{IC}=10 \mathrm{~mA}, \mathrm{IB}=1 \mathrm{~mA}$ & 803.59 & 805.86 & -2.27 & 1.58 & 1.74 & -0.16 \\
\hline $\mathrm{VCES}(\mathrm{mV}) @ \mathrm{IC}=100 \mathrm{~mA}, \mathrm{IB}=10 \mathrm{~mA}$ & 463.22 & 478.11 & -14.89 & 31.67 & 21.92 & 9.75 \\
\hline $\operatorname{VBES}(\mathrm{mV}) @ \mathrm{IC}=100 \mathrm{~mA}, I \mathrm{~B}=10 \mathrm{~mA}$ & 1129.9 & 1139.8 & .9 .9 & 8.08 & 20.04 & -11.96 \\
\hline VBEON $(\mathrm{mV}) @ \mathrm{VCE}=5 \mathrm{~V}, \mathrm{lC}=5 \mathrm{~mA}$ & 726.34 & 727.47 & -1.13 & 3.43 & 3.53 & -0.1 \\
\hline VBEON $(\mathrm{mV}) @ \mathrm{VCE}=5 \mathrm{~V}, I \mathrm{C}=1.0 \mathrm{~mA}$ & 781.41 & 782.78 & -1.37 & 5.77 & 4.48 & 1.29 \\
\hline $\mathrm{ICBO}(\mathrm{nA}) @ \mathrm{VCB}=-50 \mathrm{~V}$ & 1.5 & 0.61 & 0.89 & 0.025 & 0.0079 & 0.0171 \\
\hline $\mathrm{ICBO}(\mathrm{nA}) @ \mathrm{VCB}=-70 \mathrm{~V}$ & 1.6 & 0.68 & 0.92 & 0.12 & 0.059 & 0.061 \\
\hline $\operatorname{ICES}(\mathrm{nA}) @ \mathrm{VCE}=-50 \mathrm{~V}$ & 0.85 & 0.39 & 0.46 & 0.033 & 0.0051 & 0.0279 \\
\hline $\operatorname{ICER}(n A) @ V C=-50 V, R=1 K$ & 0.7 & 0.33 & 0.37 & 0.028 & 0.0071 & 0.0209 \\
\hline $\operatorname{IEBO}(\mathrm{nA}) @$ VEB $=-5 \mathrm{~V}$ & 0.005 & 0.0075 & -0.003 & 0 & 0.0065 & -0.0065 \\
\hline $\operatorname{VCES}(\mathrm{mV}) @ \mathrm{IC}=-10 \mathrm{~mA}, \mathrm{IB}=-500 \mu \mathrm{A}$ & 82.28 & 85.19 & -2.91 & 0.752 & 0.689 & 0.063 \\
\hline $\mathrm{VBES}(\mathrm{mV}) @ \mathrm{IC}=-10 \mathrm{~mA}, \mathrm{IB}=-500_{\mu} \mathrm{A}$ & 764.56 & 767.42 & -2.86 & 0.403 & 0.575 & -0.172 \\
\hline $\mathrm{VBEON}(\mathrm{mV}) @ V C E=-5 \mathrm{~V}, \mathrm{IC}=-5 \mathrm{~mA}$ & 705.09 & 705.03 & 0.06 & 0.523 & 0.776 & -0.253 \\
\hline VBEON $(\mathrm{mV}) @ \mathrm{VCE}=-5 \mathrm{~V}, \mathrm{IC}=-20 \mathrm{~mA}$ & 748.16 & 747.78 & 0.38 & 0.397 & 0.624 & -0.227 \\
\hline $1+\mathrm{HFE} @ \mathrm{VCB}=-5 \mathrm{~V}, \mathrm{IE}=-10 \mu \mathrm{A}$ & 217.25 & 243.5 & -26.25 & 3.9412 & 4.5794 & -0.638 \\
\hline HFE @VCE $=-5 \mathrm{~V}, \mathrm{IC}=-500 \mu \mathrm{A}$ & 210.81 & 235.83 & -25.02 & 3.6918 & 4.5407 & -0.849 \\
\hline $\mathrm{HFE} @ \mathrm{VCE}=-5 \mathrm{~V}, \mathrm{IC}=-10 \mathrm{~mA}$ & 197.54 & 220.17 & -22.63 & 3.4727 & 4.033 & -0.56 \\
\hline HFE @ VCE $=-5 \mathrm{~V}, I \mathrm{C}=-20 \mathrm{~mA}$ & 185.05 & 206.44 & .21 .39 & 3.1123 & 3.6335 & -0.521 \\
\hline IR $(n A) @ V R=55 V$ & 13 & 13 & 0 & 1.3 & 1.7 & -0.4 \\
\hline $\mathrm{VF}(\mathrm{mV}) @ \mathrm{IF}=1 \mathrm{~mA}$ & 628 & 630.92 & -2.92 & 2.06 & 2.2 & -0.14 \\
\hline $\mathrm{VF}(\mathrm{mV}) @ \mathrm{IF}=10 \mathrm{~mA}$ & 757.56 & 760.47 & -2.91 & 3.45 & 3.32 & 0.13 \\
\hline $\mathrm{ICBO}(\mathrm{nA}) @ \mathrm{VCB}=-50 \mathrm{~V}$ & 1.6 & 0.78 & 0.82 & 0 & 0.0067 & -0.0067 \\
\hline $\mathrm{ICBO}(\mathrm{nA}) @ \mathrm{VCB}=-70 \mathrm{~V}$ & 1.6 & 0.76 & 0.84 & 0.055 & 0.037 & 0.018 \\
\hline $\mathrm{ICES}(\mathrm{nA}) @ \mathrm{VCE}=-50 \mathrm{~V}$ & 0.88 & 0.48 & 0.4 & 0.011 & 0.0038 & 0.0072 \\
\hline $\mathrm{ICER}(\mathrm{nA}) @ \mathrm{VC}=-50 \mathrm{~V}, \mathrm{R}=1 \mathrm{~K}$ & 0.68 & 0.39 & 0.29 & 0.014 & 0.0062 & 0.0078 \\
\hline $\mathrm{IEBO}(\mathrm{nA}) @ \mathrm{VEB}=-5 \mathrm{~V}$ & 0.005 & 0.036 & -0.031 & 0 & 0.009 & -0.009 \\
\hline $\begin{array}{l}\text { VCES }(\mathrm{mV}) @ \mathrm{IC}=-10 \mathrm{~mA}, I B=- \\
500 \mu \mathrm{A}\end{array}$ & 81.44 & 84.42 & -2.98 & 0.629 & 0.522 & 0.107 \\
\hline
\end{tabular}




\begin{tabular}{|c|c|c|c|c|c|c|}
\hline \multirow[t]{2}{*}{ TEST } & \multicolumn{3}{|c|}{ AVERAGE VALUES } & \multicolumn{3}{|c|}{ STANDARD DEVIATION } \\
\hline & Freon & DI Water & Delta & Freon & DI Water & Delta \\
\hline VBES $(\mathrm{mV}) @ \mathrm{IC}=-10 \mathrm{~mA}, \mathrm{IB}=-500 \mu \mathrm{A}$ & 764.81 & 767.69 & -2.88 & 0.359 & 0.598 & -0.239 \\
\hline VBEON $(\mathrm{mV}) @ \mathrm{VCE}=-5 \mathrm{~V}, \mathrm{IC}=-5 \mathrm{~mA}$ & 704.88 & 705 & $.0,12$ & 0.465 & 0.786 & -0.321 \\
\hline VBEON $(\mathrm{mV}) @ \mathrm{VCE}=-5 \mathrm{~V}, \mathrm{IC}=-20 \mathrm{mIA}$ & 744.72 & 747.64 & -2.92 & 0.632 & 0.782 & -0.15 \\
\hline $1+\mathrm{HFE} @ \mathrm{VCB}=-5 \mathrm{~V}, \mathrm{IE}=-10 \mu \mathrm{A}$ & 229.94 & 254.67 & -24.73 & 4.9996 & 1.5339 & 3,4657 \\
\hline HFE Q VCE $=-5 \mathrm{~V}, \mathrm{IC}=-500 \mu \mathrm{A}$ & 223.38 & 246.67 & -2.3 .29 & 4.8973 & 1.4951 & 3.4022 \\
\hline $\mathrm{HFE} @ \mathrm{VCE}=-5 \mathrm{~V}, \mathrm{IC}=-10 \mathrm{~mA}$ & 209.13 & 229.94 & -20.81 & 4.1932 & 1,3921 & 2.8011 \\
\hline HFE@VCE $=-5 \mathrm{~V}, \mathrm{IC}=-20 \mathrm{~mA}$ & 195.64 & 215.39 & -19.75 & 3.8196 & 1.195 & 2.6246 \\
\hline $\mathrm{ICBO}(\mathrm{nA}) @ \mathrm{VCB}=20 \mathrm{~V}$ & 3.3 & 2.6 & 0.7 & 0.41 & 0.25 & 0.16 \\
\hline IEBO $(n A) @ V E B=4 V$ & 27 & 19 & 8 & 24 & 11 & 13 \\
\hline $\operatorname{ICER}(\mathrm{nA}) @ \mathrm{VCE}=13 \mathrm{~V}, \mathrm{R}=1 \mathrm{~K}$ & 2.6 & 2 & 0.6 & 0.25 & 0.075 & 0.175 \\
\hline HFE @ VCE $=5 \mathrm{~V}, I \mathrm{C}=1 \mathrm{~mA}$ & 92.844 & 65.339 & 27.505 & 23.511 & $10.3 ! 6$ & 13.195 \\
\hline $\mathrm{HFE} @ \mathrm{VCE}=5 \mathrm{~V}, \mathrm{IC}=10 \mathrm{~mA}$ & 116.16 & 84.794 & 31.366 & 26.646 & 12.189 & 14.457 \\
\hline HFE @ VCE $=5 \mathrm{~V}, \mathrm{IC}=100 \mathrm{~mA}$ & 83.25 & 64.489 & 18.761 & 16.479 & 7.8785 & 8.6005 \\
\hline VCES $(\mathrm{mV}) @ \mathrm{IC}=10 \mathrm{~mA}, \mathrm{IB}=1 \mathrm{~mA}$ & 138.16 & 147.33 & -9.17 & 5,03 & 4,13 & 0.9 \\
\hline VBES $(m \mathrm{~m}) @ \mathrm{IC}=10 \mathrm{~mA}, \mathrm{IB}=1 \mathrm{~mA}$ & 802.41 & 805.69 & $-3,28$ & 2.12 & 1.1 & 1.02 \\
\hline $\operatorname{VCES}(\mathrm{mV})(2) \mathrm{IC}=100 \mathrm{~mA}, \mathrm{IB}=10 \mathrm{~mA}$ & 436.38 & 494.42 & -58.04 & 26.75 & 22.87 & 3.88 \\
\hline VBES $(\mathrm{mV}) @ I C=100 \mathrm{~mA}, I \mathrm{IB}=10 \mathrm{~mA}$ & 1129.8 & 1126.4 & 3.4 & 11.19 & 12.84 & -1.55 \\
\hline $\mathrm{VBEON}(\mathrm{mV}) @ \mathrm{VCE}=5 \mathrm{~V}, \mathrm{IC}=5 \mathrm{~mA}$ & 723.38 & 730.25 & -6.87 & 4.18 & 2.91 & 1.27 \\
\hline VBEON $(\mathrm{mV}) @ \mathrm{VCE}=5 \mathrm{~V}, I \mathrm{C}=20 \mathrm{~mA}$ & 776.97 & 785.83 & .8 .86 & 6.51 & 3.91 & 2.6 \\
\hline $\mathrm{ICBO}(\mathrm{nA}) @ \mathrm{VCB}=-50 \mathrm{~V}$ & 1.3 & 0.66 & 0.64 & 0 & 0.0084 & -0.0084 \\
\hline $\mathrm{ICBO}(\mathrm{nA}) @ \mathrm{VCB}=-70 \mathrm{~V}$ & 1.5 & 0.77 & 0.73 & 0.064 & 0.039 & 0.025 \\
\hline ICES (nA)@VCE $=-50 \mathrm{~V}$ & 0.77 & 0.43 & 0.34 & 0.045 & 0.014 & 0.031 \\
\hline $\operatorname{ICER}(\mathrm{n}, \mathrm{A})(\mathrm{g}) \mathrm{VC}=-50 \mathrm{~V}, \mathrm{R}=1 \mathrm{~K}$ & 0.65 & 0.36 & 0.29 & 0.01 & 0.007 & 0.003 \\
\hline $\operatorname{IEBO}(\mathrm{nA}) @ \mathrm{VEB}=-5 \mathrm{~V}$ & 0.005 & 0.005 & 0 & 0 & 0 & 0. \\
\hline VCES $(\mathrm{mV}) @ \mathrm{IC}=-10 \mathrm{~mA}, \mathrm{IB}=-500 \mu \mathrm{A}$ & 81.94 & 85.72 & -3.78 & 0.727 & 0.79 & -0.063 \\
\hline VBES (mV)@IC $=-10 \mathrm{~mA}, I B=-500 \mu \mathrm{A}$ & 764.59 & 767.69 & -3.1 & 0.417 & 0.489 & -0.072 \\
\hline VBEON $(\mathrm{mV}) @ \mathrm{VCE}=-5 \mathrm{~V}, \mathrm{IC}=-5 \mathrm{~mA}$ & 704.78 & 705.08 & -0.3 & 0.547 & 0.827 & -0.28 \\
\hline VBEON $(\mathrm{mV}) @ \mathrm{VCE}=-5 \mathrm{~V}, \mathrm{IC}=-20 \mathrm{~mA}$ & 747.81 & 747.94 & -0.13 & 0.443 & 0.662 & -0.219 \\
\hline $1+\mathrm{HFE} @ \mathrm{VCB}=-5 \mathrm{~V}, \mathrm{IE}=-10 \mu \mathrm{A}$ & 220.25 & 242.39 & -22.14 & 3.8557 & 3.0125 & 0.8432 \\
\hline HFE @ VCE $=-5 \mathrm{~V}, I \mathrm{C}=-500 \mu \mathrm{A}$ & 213.5 & 235.06 & .21 .56 & 3.5777 & 2.6892 & 0.8885 \\
\hline HFE @ YCE $=-5 \mathrm{~V}, \mathrm{IC}=-10 \mathrm{~mA}$ & 199.78 & 219.44 & -19.66 & 3.2196 & 2.5257 & 0.6939 \\
\hline $\mathrm{HFE} @ \mathrm{VCE}=-5 \mathrm{~V}, \mathrm{IC}=-20 \mathrm{~mA}$ & 187.01 & 205.67 & .18 .60 & 2.7325 & 2.3764 & 0.3561 \\
\hline
\end{tabular}




\begin{tabular}{|c|c|c|c|c|c|c|}
\hline \multirow[t]{2}{*}{ TEST } & \multicolumn{3}{|c|}{ MINIMUM VALUES } & \multicolumn{3}{|c|}{ MAXIMUM VALUES } \\
\hline & Freon & DI Water & Delta & Freon & DI Water & Delta \\
\hline $\mathrm{IR}(\mathrm{nA}) @ \mathrm{VR}=55 \mathrm{~V}$ & 12 & 11 & 1 & 20 & 19 & 1 \\
\hline$V F(m V) @ I F=1 \mathrm{~mA}$ & 615.5 & 620 & -4.5 & 627 & 632 & .5 \\
\hline $\mathrm{VF}(\mathrm{mV}) @ \mathrm{IF}=10 \mathrm{~mA}$ & 745.5 & 748.5 & -3 & 759 & 764 & -5 \\
\hline $\mathrm{ICBO}(\mathrm{nA}) @ \mathrm{VCB}=-50 \mathrm{~V}$ & 0.8 & 0.79 & 0.01 & 0.82 & 0.81 & 0.01 \\
\hline $\mathrm{ICBO}(\mathrm{nA}) @ \mathrm{VCB}=-70 \mathrm{~V}$ & 0.75 & 0.74 & 0.01 & 0.85 & 0.75 & 0.1 \\
\hline$I C E S(n A) @ V C E=-50 V$ & 0.48 & 0.46 & 0.02 & 0,48 & 0.48 & 0 \\
\hline $\operatorname{ICER}(\mathrm{nA}) @ V C=-50 \mathrm{~V}, \mathrm{R}=1 \mathrm{~K}$ & 0.36 & 0.36 & 0 & 0.4 & 0.4 & 0 \\
\hline IEBO (nA)@VEB $=-5 \mathrm{~V}$ & 0.005 & 0.025 & -0.02 & 0.015 & 0.05 & $-0,035$ \\
\hline $\begin{array}{l}\text { VCES }(\mathrm{mV}) @ \mathrm{IC}=-10 \mathrm{~mA}, \mathrm{IB}=- \\
500 \mu \mathrm{A}\end{array}$ & 80.5 & 83.5 & -3 & 84.5 & 85.5 & -1 \\
\hline VBES (mV)@IC $=-10 \mathrm{~mA}, \mathrm{IB}=-500 \mu \mathrm{A}$ & 759 & 764 & -5 & 764.5 & 768 & .3 .5 \\
\hline $\mathrm{VBEON}(\mathrm{mV}) @ \mathrm{VCE}=-5 \mathrm{~V}, \mathrm{IC}=-5 \mathrm{~mA}$ & 697 & 701 & -4 & 704.5 & 705.5 & -1 \\
\hline VBEON (mV)@ VCE $=-5 \mathrm{~V}, \mathrm{IC}=-20 \mathrm{~mA}$ & 740.5 & 744 & -3.5 & 748 & 748 & 0 \\
\hline $1+\mathrm{HFE} @ \mathrm{VCB}=-5 \mathrm{~V}, \mathrm{IE}=-10 \mu \mathrm{A}$ & 221 & 250 & .29 & 238 & 258 & .20 \\
\hline $\mathrm{HFE} @ \mathrm{VCE}=-5 \mathrm{~V}, \mathrm{IC}=-500 \mu \mathrm{A}$ & 215 & 243 & .28 & 121 & 250 & -129 \\
\hline HFE $Q$ VCE $=-5 \mathrm{~V}, I \mathrm{C}=-10 \mathrm{~mA}$ & 201 & 227 & -26 & 216 & 233 & -17 \\
\hline $\mathrm{HFE} @ \mathrm{VCE}=-5 \mathrm{~V}, \mathrm{IC}=20 \mathrm{~mA}$ & 188.8 & 212 & -23.2 & 203 & 219 & -16 \\
\hline $1 \mathrm{CBO}(\mathrm{nA}) @ \mathrm{VCB}=20 \mathrm{~V}$ & 3.3 & 2.6 & 0.7 & 5.6 & 4 & 1.6 \\
\hline IEBO (nA)@ VEB =4V & 13 & 12 & 1 & 143 & 64 & 79 \\
\hline $\operatorname{ICER}(\mathrm{nA}) @ \mathrm{VCE}=13 \mathrm{~V}, \mathrm{R}=1 \mathrm{~K}$ & 2.7 & 2.1 & 0.6 & 3.7 & 2.6 & 1.1 \\
\hline $\mathrm{HFE} @ \mathrm{VCE}=5 \mathrm{~V}, \mathrm{IC}=1 \mathrm{~mA}$ & 53.4 & 54 & -0.6 & 102 & 89.5 & 12.4 \\
\hline HFE $Q V C E=5 \mathrm{~V}, \mathrm{IC}=10 \mathrm{~mA}$ & 71.7 & 70.9 & 0.8 & 131.2 & 112.9 & 18.3 \\
\hline HFE @ VCE $=5 \mathrm{~V}, \mathrm{IC}=100 \mathrm{~mA}$ & 54.7 & 55.3 & -0.6 & 93.3 & 82.7 & 10.6 \\
\hline $\mathrm{VCES}(\mathrm{mV}) @ \mathrm{IC}=10 \mathrm{~mA}, \mathrm{IB}=1 \mathrm{~mA}$ & 140.5 & 139 & 1.5 & 152 & 153.5 & -1.5 \\
\hline $\mathrm{VBES}(\mathrm{mV}) @ I \mathrm{C}=10 \mathrm{~mA}, I \mathrm{IB}=1 \mathrm{~mA}$ & 796.5 & 800.5 & -4 & .803 .5 & 807 & -3.5 \\
\hline VCES $(\mathrm{mV}) @ I \mathrm{C}=100 \mathrm{~mA}, I B=10 \mathrm{~mA}$ & 441.5 & 428.5 & 13 & 519 & 520.5 & -1.5 \\
\hline VBES $(\mathrm{mV}) @ \mathrm{IC}=100 \mathrm{~mA}, \mathrm{IB}=10 \mathrm{~mA}$ & 1119 & 1117.5 & 1.5 & 1147.5 & 1179 & -31.5 \\
\hline $\mathrm{VBEON}(\mathrm{mV}) @ \mathrm{VCE}=5 \mathrm{~V}, \mathrm{IC}=5 \mathrm{~mA}$ & 714.5 & 719.5 & -5 & 728.5 & 732 & -3.5 \\
\hline $\mathrm{VBEON}(\mathrm{mV}) @ \mathrm{VCE}=5 \mathrm{~V}, I \mathrm{C}=10 \mathrm{~mA}$ & 767 & 773 & -6 & 787 & 789 & -2 \\
\hline $\mathrm{ICBO}(\mathrm{nA}) @ \mathrm{VCB}=-50 \mathrm{~V}$ & 0.61 & 0.6 & 0.01 & 0.63 & 0.61 & 0.02 \\
\hline $\mathrm{ICBO}(\mathrm{nA}) @ \mathrm{VCB}=-70 \mathrm{~V}$ & 0.65 & 0.6 & 0.05 & 0.75 & 0.7 & 0.05 \\
\hline ICES (nA)@VCE $=-50 \mathrm{~V}$ & 0.38 & 0.38 & 0 & 0.4 & 0.4 & 0 \\
\hline $\operatorname{ICER}(\mathrm{nA}) @ V C=-50 \mathrm{~V}, \mathrm{R}=1 \mathrm{~K}$ & 0,32 & 0.32 & 0 & 0.33 & 0.34 & -0.01 \\
\hline $\mathrm{IEBO}(\mathrm{nA}) @ V E B=-5 \mathrm{~V}$ & 0.005 & 0.005 & 0 & 0,005 & 0.035 & .0 .03 \\
\hline VCES $(\mathrm{mV}) @ \mathrm{IC}=-10 \mathrm{~mA}, \mathrm{IB}=-500 \mu \mathrm{A}$ & 81.5 & 84 & -2.5 & 83.5 & 86 & -2.5 \\
\hline VBES $(\mathrm{mV}) @ \mathrm{IC}=-10 \mathrm{~mA}, \mathrm{IB}=-500 \mu \mathrm{A}$ & 758 & 764 & -6 & 764 & 768 & -4 \\
\hline VBEON(mV)@VCE $=-5 \mathrm{~V}, \mathrm{IC}=-5 \mathrm{~mA}$ & 696.5 & 701.5 & -5 & 704 & 705 & -2 \\
\hline VBEON $(\mathrm{mV}) @ \mathrm{VCE}=-5 \mathrm{~V}, \mathrm{IC}=-20 \mathrm{~mA}$ & 740 & 744.5 & .4 .5 & 747.5 & 748.5 & -1 \\
\hline $1+\mathrm{HFE} @ \mathrm{VCB}=-5 \mathrm{~V}, \mathrm{IE}=-10 \mu \mathrm{A}$ & 210 & 236 & -26 & 227 & 247 & -20 \\
\hline HFE @ VCE $=-5 \mathrm{~V}, \mathrm{IC}=-500 \mu \mathrm{A}$ & 204 & 229 & -25 & 220 & 239 & -19 \\
\hline $\mathrm{HFE} @ \mathrm{VCE}=-5 \mathrm{~V}, \mathrm{IC}=-10 \mathrm{~mA}$ & 191.6 & 214 & -22.4 & 206 & 223 & -17 \\
\hline $\mathrm{HFE} @ \mathrm{VCE}=-5 \mathrm{~V}, \mathrm{IC}=-20 \mathrm{~mA}$ & 179.9 & 201 & -21.1 & 192.8 & 209 & -16.2 \\
\hline $\mathrm{IR}(\mathrm{nA}) @ \mathrm{VR}=55 \mathrm{~V}$ & 12 & 12 & 0 & 20 & 17 & 3 \\
\hline $\mathrm{VF}(\mathrm{mV}) @ \mathrm{IF}=1 \mathrm{~mA}$ & 616.5 & 624.5 & .8 & 625.5 & 632 & -6.5 \\
\hline $\mathrm{VF}(\mathrm{mV}) @ \mathrm{IF}=10 \mathrm{~mA}$ & 745.5 & 754 & -8.5 & 756 & 762 & -6 \\
\hline $\mathrm{ICBO}(\mathrm{nA}) @ \mathrm{VCB}=-50 \mathrm{~V}$ & 0.78 & 0.76 & 2.02 & 0.8 & 0.87 & -0.07 \\
\hline $\mathrm{ICBO}(\mathrm{nA}) @ \mathrm{VCB}=-70 \mathrm{~V}$ & 0.75 & 0.7 & 0.05 & 0.8 & 0.8 & 0 \\
\hline $\operatorname{ICES}(\mathrm{nA}) @ V C E=-50 \mathrm{~V}$ & 0.48 & 0.48 & 0 & 0.49 & 0.57 & 0.08 \\
\hline $\operatorname{ICER}(\mathrm{nA}) @ \mathrm{VC}=-50 \mathrm{~V}, \mathrm{R}=1 \mathrm{~K}$ & 0.36 & 0.38 & $-0,02$ & 0.4 & 0.48 & 0.08 \\
\hline IEBO $(n A) @ V E B=-5 V$ & 0.005 & 0.015 & -0.01 & 0.005 & 0.14 & -0.135 \\
\hline $\begin{array}{l}\text { VCES }(\mathrm{mV}) @ \mathrm{IC}=-10 \mathrm{~mA}, \mathrm{IB}=- \\
500 \mu \mathrm{A}\end{array}$ & 80.5 & 83.5 & -3 & 83.5 & 85 & -1.5 \\
\hline
\end{tabular}




\begin{tabular}{|c|c|c|c|c|c|c|}
\hline \multicolumn{7}{|c|}{$\begin{array}{l}\text { TABLE A31: MINIMUM AND MAXIMUM VALUES OF POST } 500 \text { HOUR HTRB ELECTRICAL TEST DATA } \\
\text { FOR M-50 (CONTINUED) }\end{array}$} \\
\hline TEST & \multicolumn{3}{|c|}{ MINIMUM VALUES } & \multicolumn{3}{|c|}{ MAXIMUM VALUES } \\
\hline & Freon & DI Water & Delta & Freon & DI Water & Delta \\
\hline VBES $(\mathrm{mV}) @ I C=-10 \mathrm{~mA}, I B=-500 \mu \mathrm{A}$ & 759 & 764.5 & -5.5 & 764.5 & 768 & -3.5 \\
\hline $\mathrm{VBEON}(\mathrm{mV}) @ V C E=-5 \mathrm{~V}, I \mathrm{C}=-5 \mathrm{~mA}$ & 697.5 & 702 & -4.5 & 704.5 & 705.5 & -1 \\
\hline $\mathrm{VBEON}(\mathrm{mV}) @ \mathrm{VCE}=-5 \mathrm{~V}, \mathrm{IC}=-20 \mathrm{~mA}$ & 740.5 & 744.5 & .4 & 747.5 & 748.5 & .1 \\
\hline $1+\mathrm{HFE} @ \mathrm{VCB}=-5 \mathrm{~V}, \mathrm{IE}=-10 \mu \mathrm{A}$ & 218 & 247 & .29 & 239 & 259 & -20 \\
\hline $\mathrm{HFE} @ \mathrm{VCE}=-5 \mathrm{~V}, \mathrm{IC}=-500 \mu \mathrm{A}$ & 218 & 238 & .20 & 232 & 259 & -20 \\
\hline $\mathrm{HFE} @ V C E=-5 \mathrm{~V}, I \mathrm{C}=.10 \mathrm{~mA}$ & 206 & 222 & .16 & 216 & 234 & -18 \\
\hline $\mathrm{HFE} @ \mathrm{VCE}=-5 \mathrm{~V}, \mathrm{IC}=-20 \mathrm{~mA}$ & 192.8 & 209 & -16.2 & 202 & 219 & -17 \\
\hline$I C B O(n A) @ V C B=20 V$ & 3 & 2.5 & 0.5 & 5 & 4.4 & $\overline{0.6}$ \\
\hline $\mathrm{IEBO}(\mathrm{nA})(\mathrm{VEB}=4 \mathrm{~V}$ & 14 & 13 & 1 & 188 & 122 & 66 \\
\hline $\operatorname{ICER}(\mathrm{nA}) @ \mathrm{VCE}=13 \mathrm{~V}, \mathrm{R}=1 \mathrm{~K}$ & 2.3 & 2.1 & 0.2 & 3,6 & 2.6 & 1 \\
\hline $\mathrm{HFE} @ \mathrm{VCE}=5 \mathrm{~V}, \mathrm{lC}=1 \mathrm{~mA}$ & 58.2 & 55.6 & 2.6 & 120.2 & 82.1 & 38.1 \\
\hline $\mathrm{HFE} @ \mathrm{VCE}=5 \mathrm{~V}, \mathrm{IC}=10 \mathrm{~mA}$ & 76.7 & 72.9 & 3.8 & 145,8 & 105.5 & 40.3 \\
\hline $\mathrm{HFE} @ \mathrm{VCE}=5 \mathrm{~V}, I \mathrm{IC}=100 \mathrm{~mA}$ & 59 & 56.6 & $2: 4$ & 101.1 & 77,9 & 23.2 \\
\hline $\mathrm{VCES}(\mathrm{mV}) @ \mathrm{IC}=10 \mathrm{~mA}, \mathrm{IB}=1 \mathrm{~mA}$ & 134 & 140.5 & .6 .5 & 150.5 & 152 & -1.5 \\
\hline VBES $(\mathrm{mV}) @ I \mathrm{C}=10 \mathrm{~mA} \cdot \mathrm{IB}=1 \mathrm{~mA}$ & 793.5 & 801.5 & -8 & 803 & 806.5 & -3.5 \\
\hline $\operatorname{VCES}(\mathrm{mV}) @ \mathbb{I C}=100 \mathrm{~mA}, \mathrm{IB}=10 \mathrm{~mA}$ & 414 & 460 & -46 & 512 & 521 & .9 \\
\hline VBES $(\mathrm{mV}) @ I C=100 \mathrm{~mA}, I \mathrm{~B}=10 \mathrm{~mA}$ & 1115 & 1115.5 & -0.5 & 1146 & 1154.5 & -8.5 \\
\hline VBEON $(m \mathrm{~V}) @ \mathrm{VCE}=5 \mathrm{~V}, \mathrm{IC}=5 \mathrm{~mA}$ & 711 & 720.5 & .9 .5 & 726 & 732 & -6 \\
\hline VBEON $(\mathrm{mV}) @ \mathrm{VCE}=5 \mathrm{~V}, \quad I \mathrm{C}=20 \mathrm{~mA}$ & 763 & 774.5 & -11.5 & 783 & 789.5 & .0 .5 \\
\hline $\mathrm{ICBO}(\mathrm{nA}) @ \mathrm{VCB}=-50 \mathrm{~V}$ & 0.66 & 0.66 & 0 & 0.69 & 0.68 & 0.01 \\
\hline $1 \mathrm{CBO}(\mathrm{nA}) @ \mathrm{VCB}=.70 \mathrm{~V}$ & 0.75 & 0.7 & 0.05 & 0.85 & 0.8 & 0.05 \\
\hline $\operatorname{ICES}(\mathrm{nA}) @ V C E=-50 \mathrm{~V}$ & 0.4 & 0.4 & 0 & 0.44 & 0,46 & -0.02 \\
\hline ICER $(\mathrm{nA}) @ V C=-50 \mathrm{~V}, \mathrm{R}=1 \mathrm{~K}$ & 0.34 & $0, \overline{34}$ & 0 & 0.37 & 0,36 & 0.01 \\
\hline $\mathrm{IEBO}(\mathrm{nA}) @ \mathrm{VEB}=-5 \mathrm{~V}$ & 0.005 & 0.005 & 0 & 0.005 & 0.005 & 0 \\
\hline $\mathrm{VCES}(\mathrm{mV}) @ \mathrm{IC}=-10 \mathrm{~mA}, \mathrm{IB}=-500 \mu \mathrm{A}$ & 81.5 & 84.5 & .3 & 84 & 86 & -2 \\
\hline $\mathrm{VBES}(\mathrm{mV}) @ \mathrm{IC}=-10 \mathrm{~mA}, \mathrm{IB}=-500 \mu \mathrm{A}$ & 758 & 764.5 & -6.5 & 764.5 & 768 & .3 .5 \\
\hline VBEON $(m V) @ V C E=-5 V, I C=-5 m A$ & 696.5 & 702 & -5.5 & 704.5 & 706 & .1 .5 \\
\hline $\mathrm{VBEON}(\mathrm{mV}) @ \mathrm{VCE}=-5 \mathrm{~V}, I \mathrm{C}=-20 \mathrm{~mA}$ & 740.5 & 744.5 & -4 & 747.5 & 749 & -1.5 \\
\hline $1+\mathrm{HFE} @ \mathrm{VCB}=-5 \mathrm{~V}, \mathrm{IE}=-10 \mu \mathrm{A}$ & 213 & 235 & -22 & 225 & 246 & .21 \\
\hline $\mathrm{HFE} @ \mathrm{VCE}=-5 \mathrm{~V}, \mathrm{IC}=-500 \mu \mathrm{A}$ & 207 & 227 & -20 & 219 & 238 & -19 \\
\hline HFE $@ V C E=-5 V, I C=-10 \mathrm{~mA}$ & 193.8 & 213 & -19.2 & 205 & 223 & -18 \\
\hline HFE @ VCE $=-5 \mathrm{~V}, \mathrm{IC}=-20 \mathrm{~mA}$ & 181.8 & 199.6 & -17.8 & 191.5 & 209 & -17.5 \\
\hline
\end{tabular}




\begin{tabular}{|c|c|c|c|c|c|c|}
\hline \multirow[t]{2}{*}{ TEST } & \multicolumn{3}{|c|}{ AVERAGE VALUES } & \multicolumn{3}{|c|}{ STAND ARD DEVIATION } \\
\hline & Freon & DI Water & Delta & Freon & DI Water & Delta \\
\hline$I R(n A) @ V R=55 \mathrm{~V}$ & 16 & 15 & 1 & 1.9 & 2.4 & .0 .9 \\
\hline$V F(m V) @ I F=1 \mathrm{~mA}$ & 620.5 & 626.28 & -5.78 & 3,09 & 2.99 & 0.1 \\
\hline $\mathrm{VF}(\mathrm{mV}) @ \mathrm{IF}=10 \mathrm{~mA}$ & 750.55 & 755.9 & .5 .35 & 4.36 & 3.84 & 0.52 \\
\hline $\mathrm{ICBO}(\mathrm{nA}) Q \mathrm{VCB}=-50 \mathrm{~V}$ & 0.81 & 0.8 & 0.01 & 0.0071 & 0,0032 & 0.0039 \\
\hline ICBO $(n \mathrm{~A}) @$ VCB $=-70 \mathrm{~V}$ & 0,79 & 0.77 & 0.02 & 0.028 & 0.024 & 0.004 \\
\hline$I C E S(n A) \otimes V C E=-50 V$ & 0.48 & 0.47 & 0.01 & 0 & 0,0047 & .0 .0047 \\
\hline$I C E R(n A) @ V C=-50 V, R=I K$ & 0.37 & 0.39 & .0 .02 & 0.015 & 0.012 & 0.003 \\
\hline IEBO $(n A) @ V E B=-5 V$ & 0.006 & 0.037 & -0.031 & 0,0026 & 0.006 & -0.003 \\
\hline $\begin{array}{l}\mathrm{VCES}(\mathrm{mV}) @ \mathrm{IC}=.10 \mathrm{~mA}, \mathrm{IB}=4 \\
500 \mu \mathrm{A}\end{array}$ & 82.08 & 84.33 & -2.25 & 0.963 & 0.52 & 0.443 \\
\hline VBES $(\mathrm{mV}) @ \mathrm{IC}=-10 \mathrm{~mA},[\mathrm{~B}=-500 \mu \mathrm{A}$ & 760.65 & 765.7 & .5 .05 & 1.61 & 1,37 & 0,24 \\
\hline VBEON $(m V) @ V C E=-5 V, I C=-5 \mathrm{~mA}$ & 699.68 & 703 & .3 .32 & 2.24 & 1.35 & 0.89 \\
\hline VBEON $(\mathrm{mV}) @ V C E=.5 \mathrm{~V}, \mathrm{C}=-20 \mathrm{~mA}$ & 742.95 & 745.6 & .2 .65 & 2.09 & 1.3 & 0.79 \\
\hline $1+\mathrm{HFE} @ \mathrm{VCB}=-5 \mathrm{~V}, \mathrm{IE}=-10 \mu \mathrm{A}$ & 230.35 & 255.2 & .24 .85 & 5.2643 & 2.6278 & 2.6365 \\
\hline HFE $@ V C E=-5 V, I C=-500 \mu \mathrm{A}$ & 224.05 & 246.9 & .22 .85 & 4.9148 & 2.49 & 2.4248 \\
\hline HFE @VCE $=-5 \mathrm{~V}, I \mathrm{C}=-10 \mathrm{~mA}$ & 209,75 & 230.4 & .20 .65 & 4.2162 & 2.21 & 2.0062 \\
\hline $\mathrm{HPE}(2) \mathrm{VCE}=-5 \mathrm{~V}, \mathrm{IC}=-20 \mathrm{~mA}$ & 196.45 & 215,95 & -19.5 & 3.9808 & 2.0641 & 1.9167 \\
\hline$I C B O(n A) @ V C B=20 \mathrm{~V}$ & 4.2 & 3 & 1.2 & 0.57 & 0.29 & 0.28 \\
\hline IEBO $(\mathrm{nA})$ VEB $\approx 4 \mathrm{~V}$ & 32 & 17 & 15 & 36 & 12 & 24 \\
\hline $\operatorname{ICER}(\mathrm{nA}) @ \mathrm{VCE}=13 \mathrm{~V}, \mathrm{R}=1 \mathrm{~K}$ & 3.3 & 2.4 & 0.9 & 0.32 & 0.14 & 0.18 \\
\hline $\mathrm{HFE} @ \mathrm{VCE} \approx 5 \mathrm{~V}, \mathrm{IC}=1 \mathrm{~mA}$ & 67.78 & 75.11 & -7.33 & 11.757 & 11.02 & 0.737 \\
\hline HFE (Q) VCE $\Rightarrow 5 \mathrm{~V}, I \mathrm{IC}=10 \mathrm{~mA}$ & 87.71 & 96,875 & -9.165 & 13.525 & 13.053 & 0.472 \\
\hline $\mathrm{HFE} @ \mathrm{VCE}=5 \mathrm{~V}, \mathrm{IC}=100 \mathrm{~mA}$ & 65.55 & 72.51 & -6.96 & 8.632 & 8.5292 & 0.1028 \\
\hline VCES $(\mathrm{mV}) @ I C=10 \mathrm{~mA}, \mathrm{IB}=1 \mathrm{~mA}$ & 147.1 & 143.4 & 3.7 & 2.94 & 4.53 & -1.59 \\
\hline VBES (mV)@IC $=10 \mathrm{~mA}, \mathrm{IB}=1 \mathrm{~mA}$ & 799.58 & 803.6 & $-4,02$ & 1.92 & 1.77 & 0.15 \\
\hline $\mathrm{VCES}(\mathrm{mV}) @ I C=100 \mathrm{~mA}, 1 \mathrm{~B}=10 \mathrm{~mA}$ & 486 & 471.58 & 14.42 & 21.35 & 24.89 & .3 .54 \\
\hline VEES $(\mathrm{mV}) @ \mathrm{IC}=100 \mathrm{~mA}, I B=10 \mathrm{~mA}$ & 1131.4 & $\$ 137.6$ & -6.2 & 8.12 & 17.98 & -9.86 \\
\hline VBEON $(\mathrm{mV}) @ \mathrm{VCE}=5 \mathrm{~V}, \mathrm{IC}=5 \mathrm{~mA}$ & 721,98 & 724.4 & -2.42 & 2.68 & 3.79 & -1.11 \\
\hline VBEON $(\mathrm{mV}) @ \mathrm{VCE}=5 \mathrm{~V}, \mathrm{IC}=10 \mathrm{~mA}$ & 778.45 & 779.28 & -0.83 & 3.66 & 4.75 & $-1,09$ \\
\hline$I C B O(n A) @ V C B=-50 \mathrm{~V}$ & 0.62 & 0.6 & 0.02 & 0.0074 & 0.0041 & 0.0033 \\
\hline $\mathrm{ICBO}(\mathrm{nA}) @ \mathrm{VCB}=-70 \mathrm{~V}$ & 0.69 & 0.68 & 0.01 & 0.028 & 0.03 & $-0,002$ \\
\hline ICES (nA) @ VCE $=-50 \mathrm{~V}$ & 0.39 & 0.39 & 0 & 0.0077 & $0.004 \%$ & 0.003 \\
\hline $\operatorname{ICER}(\mathrm{nA}) @ V C=-50 \mathrm{~V}, \mathrm{R}=1 \mathrm{~K}$ & 0.32 & 0.32 & 0 & 0.0033 & 0.0091 & -0.006 \\
\hline IEBO $(n A) @$ VEB $=-5 \mathrm{~V}$ & 0.005 & 0.017 & .0 .012 & 0 & 0,008 & -0.008 \\
\hline $\operatorname{VCES}(\mathrm{mV}) @ \mathrm{IC}=-10 \mathrm{~mA}, \mathrm{IB}=-500 \mu \mathrm{A}$ & 83 & 85.38 & -2.38 & 0.562 & 0.666 & -0.104 \\
\hline VBES (mV) @ IC $=-10 \mathrm{~mA}, \mathrm{IB}=-500 \mu \mathrm{A}$ & 760.3 & 765.78 & -5.48 & 1.87 & 1.35 & 0.52 \\
\hline $\mathrm{VBEON}(\mathrm{mV}) @ \mathrm{VCE}=-5 \mathrm{~V}, \mathrm{IC}=-5 \mathrm{~mA}$ & 699,68 & 703.2 & .3 .52 & 2.23 & 1.36 & 0.87 \\
\hline VBEON(mIV)@VCE $=-5 \mathrm{~V}, \mathrm{IC}=-20 \mathrm{~m} \mathrm{~A}$ & 743.1 & 745.93 & .2 .83 & 2.02 & 1,33 & 0,69 \\
\hline $1+\mathrm{HFE} @ \mathrm{VCB}=-5 \mathrm{~V}, \mathrm{IE}=-10 \mu \mathrm{A}$ & 216.25 & 241.8 & .25 .55 & 4.7559 & 3.563 & 1.1929 \\
\hline HFE @ VCE $=-5 \mathrm{~V}, \mathrm{IC}=-500 \mu \mathrm{A}$ & 210.4 & 234.4 & -24 & 4.2227 & 3.4853 & 0.7374 \\
\hline HFE @ VCE $=-5 \mathrm{~V}, \mathrm{IC}=-10 \mathrm{~mA}$ & 197.43 & 218.95 & -21.52 & 3.9651 & 2.982 & 0.9831 \\
\hline $\mathrm{HFE} @ \mathrm{VCE}=-5 \mathrm{~V}, \mathrm{IC}=-20 \mathrm{~mA}$ & 185.1 & 205.35 & .20 .25 & 3.4596 & $2 . \overline{7391}$ & 0.7205 \\
\hline $\mathrm{IR}(\mathrm{nA}) @ \mathrm{VR}=55 \mathrm{~V}$ & 17 & 14 & 3 & 2.3 & 1.7 & 0.6 \\
\hline $\mathrm{VF}(\mathrm{mV}) @ \mathrm{IF}=1 \mathrm{~mA}$ & 620.95 & 629.08 & -8.13 & 2.51 & 2 & 0.51 \\
\hline $\mathrm{VF}(\mathrm{mV}) @ \mathrm{IF}=10 \mathrm{~mA}$ & 751.98 & 759.43 & -7.45 & 3.21 & 2,16 & 1.05 \\
\hline $\mathrm{ICBO}(\mathrm{nA}) @ \mathrm{VCB}=-50 \mathrm{~V}$ & 0.79 & 0.78 & 0.01 & 0.004 & 0.022 & -0.018 \\
\hline $\mathrm{ICBO}(\mathrm{nA}) @ \mathrm{VCB}=-70 \mathrm{~V}$ & 0.77 & 0.77 & 0 & 0.0024 & 0.03 & -0.028 \\
\hline ICES $(\mathrm{nA}) @$ VCE $=-50 \mathrm{~V}$ & 0.48 & 0.418 & 0 & 0,0029 & 0.02 & -0.017 \\
\hline $\operatorname{ICER}(\mathrm{nA}) @ V C=-50 \mathrm{~V}, \mathrm{R}=1 \mathrm{~K}$ & 0.38 & 0.39 & -0.01 & 0.016 & 0.022 & -0.006 \\
\hline IEBO $(\mathrm{nA}) @$ VEB $=-5 \mathrm{~V}$ & 0.005 & 0.038 & 0.033 & 0 & 0.023 & -0.023 \\
\hline $\begin{array}{l}\mathrm{VCES}(\mathrm{mV}) @ \mathrm{IC}=-10 \mathrm{~mA}, \mathrm{IB}=- \\
500 \mu \mathrm{A}\end{array}$ & 82.43 & 84.3 & -1.87 & 0.922 & 0.377 & 0.545 \\
\hline
\end{tabular}




\begin{tabular}{|c|c|c|c|c|c|c|}
\hline \multicolumn{7}{|c|}{$\begin{array}{l}\text { TABLE A32: AVERACE AND STANDARD DEVIATION OF POST } 300 \text { HOUR HTRB ELECTRICAL TEST DATA } \\
\text { FOR M-50 (CONTINUED) }\end{array}$} \\
\hline \multirow[t]{2}{*}{ TEST } & \multicolumn{3}{|c|}{ AVERAGE VALUES } & \multicolumn{3}{|c|}{ STANDARD DEYIATION } \\
\hline & Freon & DI Water & Deita & Freon & DI Water & Deltu \\
\hline VBES $(\mathrm{mV}) @ I \mathrm{C}=-10 \mathrm{~mA}, \mathrm{IB}=-500 \mu \mathrm{A}$ & 760.55 & 766.1 & -5.55 & 1.69 & $\frac{1.18}{1.18}$ & 0.51 \\
\hline VEEON $(m \mathrm{~V})(Q V C E=-5 V, I C=-5 \mathrm{~mA}$ & 699.53 & 703.33 & $-3,8$ & 2.18 & 1,28 & 0,9 \\
\hline VBEON $(m V) @ V C E=-5 V, I C=20 m A$ & 742.78 & 746 & $-3,22$ & 2 & 1,31 & 0.69 \\
\hline $1+\mathrm{HFE} @ \mathrm{VCB}=-5 \mathrm{~V}, \mathrm{IE}=.10 \mu \mathrm{A}$ & 230.5 & 253.4 & .22 .9 & 4.9151 & 3,5748 & 1,3403 \\
\hline HFE $Q$ VCE $=-5 \mathrm{~V}, I C=-500 \mu \mathrm{A}$ & 224.25 & 245.35 & $-21,1$ & 3.9454 & 3.4985 & 0,4469 \\
\hline HFE @ VCE $=-5 \mathrm{~V}, \mathrm{IC}=-10 \mathrm{~mA}$ & 209,95 & 229 & .19 .05 & 3,4101 & 3,1119 & 0,2982 \\
\hline $\mathrm{HFE} @ \mathrm{VCE}=-5 \mathrm{~V}_{1} \mathrm{IC}=-20 \mathrm{~mA}$ & 196.53 & 214.55 & -18.02 & 3.0485 & 2.9996 & 0.0489 \\
\hline $1 \mathrm{CBO}(\mathrm{nA}) @ \mathrm{VCB}=20 \mathrm{~V}$ & 3,9 & 3.1 & 0.8 & 0.53 & 0.36 & 0.17 \\
\hline IEBO (IIA)@VEB $=4 V$ & 34 & 29 & 5 & 43 & 27 & 16 \\
\hline ICER $(n A) @ V C E=13 V, R=1 K$ & 3 & 2.3 & 0.7 & 0.38 & 0.14 & 0,24 \\
\hline HFE @ VCE $=5 \mathrm{~V}, I \mathrm{C}=1 \mathrm{~mA}$ & 90.23 & 63,045 & 27.185 & 21.281 & 6,2276 & 15.053 \\
\hline $\mathrm{HFE} @ \mathrm{VCE}=5 \mathrm{~V}, I \mathrm{C}=10 \mathrm{~mA}$ & 112.06 & 82.14 & 29,92 & 23.465 & 7.7372 & 15.728 \\
\hline HFE $@ V C E=5 V, I C=100 \mathrm{~mA}$ & 80.625 & 62.77 & 17.855 & 14.404 & 5.1791 & 9.2249 \\
\hline VCES $(\mathrm{mV})(0) \mathrm{IC}=10 \mathrm{~mA}, I \mathrm{~B}=1 \mathrm{~mA}$ & 140.5 & 148.7 & -8.2 & 4.76 & 3.05 & 1.71 \\
\hline VBES $(m V) @ I C=10 m A, I B=1 \mathrm{~mA}$ & 798,23 & 804.2 & $-5,97$ & 2.63 & 1.35 & 1,28 \\
\hline VCES $(\mathrm{mV}) @ \mathrm{IC}=100 \mathrm{~mA}, 1 \mathrm{~B}=10 \mathrm{~mA}$ & 443.73 & 498,68 & .54 .95 & 28.16 & 15,5 & 12.66 \\
\hline VBES $(m V) Q I C=100 \mathrm{~mA}, I B=10 \mathrm{~mA}$ & 1128 & 1124.4 & 3.6 & $9, \overline{34}$ & 13.23 & .3 .89 \\
\hline$V B E O N(m V) @ V C E=5 V, I C=5 \mathrm{~mA}$ & 718.88 & 728.8 & $-9,92$ & 4.24 & 3.09 & 1.15 \\
\hline VBEON $(\mathrm{mV}) @ V C E=5 \mathrm{~V}, \quad I \mathrm{C}=20 \mathrm{~mA}$ & 773.18 & 784,95 & -11.77 & 5.89 & 3.55 & 2.34 \\
\hline$I C B O(n A) @ V C B=-50 V$ & 0.67 & 0.66 & 0.01 & 0,006 & 0.0069 & -0.0009 \\
\hline$I C B O(n A) @ V C B=.70 \mathrm{~V}$ & 0.78 & 0,77 & 0.01 & 0.03 & 0,034 & -0.004 \\
\hline $\mathrm{ICES}(\mathrm{nA}) @ \mathrm{VCE}=-50 \mathrm{~V}$ & 0.41 & 0.43 & .0 .02 & 0,013 & 0.017 & .0 .004 \\
\hline $\operatorname{ICER}(\mathrm{nA}) Q V \mathrm{~V}=-50 \mathrm{~V}, \mathrm{R}=1 \mathrm{~K}$ & 0.35 & 0.35 & 0 & 0.01 & 0.0062 & 0.0038 \\
\hline $\operatorname{IEBO}(\mathrm{nA}) @ \mathrm{VEB}=-5 \mathrm{~V}$ & 0.005 & 0.005 & 0 & 0 & 0 & 0 \\
\hline$V C E S(m V) @ I C=-10 m I A, I B=-500 \mu A$ & 82.75 & 85,83 & -3.08 & 0.786 & 0,406 & 0,38 \\
\hline VBES (mV)@IC $=-10 \mathrm{~mA}, I B=-500 \mu \mathrm{A}$ & 760.33 & 766.18 & -5.85 & 1.8 & 1.21 & 0.59 \\
\hline VBEON $(\mathrm{mV}) @ V C E=-5 \mathrm{~V}, 1 \mathrm{C}=-5 \mathrm{~mA}$ & $699 . \overline{35}$ & 703.4 & -4.05 & 2.21 & 1.29 & 0,92 \\
\hline VBEON $(m V) @ V C E=-5 V, 1 C=-20 m 1 A$ & 742.7 & 746.28 & -3.58 & 1.96 & 1,26 & 0.7 \\
\hline $1+H P E @ V C B=-5 \mathrm{~V}, I E=-10 \mu \mathrm{A}$ & 220.5 & 241.4 & .20 .9 & 3,9403 & 3.0157 & 0,9246 \\
\hline $\mathrm{HFE} @ \mathrm{VCE}=-5 \mathrm{~V}, \mathrm{IC}=-500 \mu \mathrm{A}$ & 214.6 & 234.1 & -19.5 & 3.5004 & 2.7891 & 0.7113 \\
\hline $\mathrm{HFE} @ \mathrm{VCE}=.5 \mathrm{~V}, \mathrm{IC}=-10 \mathrm{~mA}$ & 201.04 & 218.85 & -17.81 & 3.2809 & 2.5189 & 0.762 \\
\hline HFE @ VCE $=-5 \mathrm{~V}, I \mathrm{C}=-20 \mathrm{~mA}$ & 188.14 & 205.23 & .17 .09 & 2.8572 & 2.3631 & 0.4941 \\
\hline
\end{tabular}


TABLE A331 MINIMUM AND MAXIMUM VALUES OF ELECTRICAL TEST DAT'A FOR M.90 AT -55 C TES'T

MINIMUM VALUE

\begin{tabular}{|c|c|c|c|c|c|c|}
\hline TEST & \multicolumn{3}{|c|}{ MINLMUM VALUE } & \multicolumn{3}{|c|}{ MAXIMUM VALUE } \\
\hline & Freon & DI Water & Doltu & Freoll & DI Water & Dolta \\
\hline$V F(m V) @ I F=I m A$ & 779.5 & 773 & 2.5 & 788.5 & 791.5 & .3 \\
\hline $\mathrm{VF}(\mathrm{mV}) \otimes I F=10 \mathrm{~mA}$ & 870 & 866 & 4 & 883.5 & 884.5 & .1 \\
\hline $1+\mathrm{HFE} @ \mathrm{QVCB}=-5 \mathrm{~V}, \mathrm{IE}=-10 \mu \mathrm{A}$ & 117.2 & 133,9 & .16 .7 & 151,9 & 146,4 & 5,5 \\
\hline HFE ( $\mathrm{V}$ VCE $=-5 \mathrm{~V}, \mathrm{IC}=-5 \mathrm{~mA}$ & 121.4 & 126.3 & $.4,9$ & 111.6 & 137,7 & .26 .1 \\
\hline HFE $@ V C E=-5 V, I C=.20 \mathrm{~mA}$ & 98,8 & 110,3 & -11.5 & 113,4 & 126 & .12 .6 \\
\hline VBEON $(\mathrm{mV}) @ V C E=-5 \mathrm{~V}, I \mathrm{C}=-5 \mathrm{~mA}$ & 843.5 & 842 & 1.5 & 848 & 848.5 & .0 .5 \\
\hline VBEON $(\mathrm{mV}) @ V C E=5 \mathrm{~V}, I \mathrm{C}=-20 \mathrm{~mA}$ & $7 \overline{92}$ & 797 & .5 & 880.5 & 878.5 & 2 \\
\hline HFE @ VCE $=5 \mathrm{~V}, I \mathrm{C} \sim 5 \mathrm{~mA}$ & 35.1 & 35,3 & -0.2 & 63,6 & 56,4 & 7.2 \\
\hline $\mathrm{HFE} @ \mathrm{VCE}=5 \mathrm{~V}, I \mathrm{C}=20 \mathrm{~mA}$ & 36 & 35.8 & 0,2 & 62.5 & 56,2 & 6.3 \\
\hline VBEON $(m \mathrm{~V}) @ \mathrm{VCE}=5 \mathrm{~V}, \mathrm{IC}=5 \mathrm{~mA}$ & 864 & 862 & 2 & 873,5 & 876 & .2 .5 \\
\hline VBEON $(\mathrm{mV}) \& \mathrm{VCE}=5 \mathrm{~V}, I \mathrm{C}=20 \mathrm{~mA}$ & 911 & 911 & 0 & 928 & 928.5 & .0 .5 \\
\hline $1+\mathrm{HFE} @ \mathrm{VCB}=-5 \mathrm{~V}, \mathrm{IE}=-10 \mu \mathrm{A}$ & 106.7 & 127.4 & -20.7 & 164,5 & 158.7 & 5.8 \\
\hline HFE @ VCE $=-5 \mathrm{~V}, \mathrm{IC}=-5 \mathrm{~mA}$ & 106.8 & 119,9 & $-13,1$ & 116,4 & 130.9 & -14.5 \\
\hline HFE $@ V C E=-5 V, I C=-20 \mathrm{~mA}$ & 100,4 & 112.3 & -11.9 & 109.1 & 122,2 & -13.1 \\
\hline VBEON $(\mathrm{mV}) \subseteq \mathrm{VCE}=-5 \mathrm{~V}, 1 \mathrm{C}=.5 \mathrm{~mA}$ & 843 & 842 & 1 & 848.5 & 849 & .0 .5 \\
\hline VBEON $(\mathrm{mV}) @ V C E=7.5 \mathrm{~V}, \mathrm{IC}=-20 \mathrm{~mA}$ & 875.5 & 874.5 & $T$ & 881 & 881 & 0 \\
\hline$V F(m V) @ I F=\ln A$ & 479.5 & 779 & 0.5 & 788.5 & 790 & -1.5 \\
\hline$V F(m V) @ I F=10 \mathrm{~mA}$ & 873.5 & 872 & 1.5 & 883.5 & 884.5 & .1 \\
\hline $1+\mathrm{HFE}(\mathrm{Q} V \mathrm{CB}=-5 \mathrm{~V}, \mathrm{IE}=-10 \mu \mathrm{A}$ & 119.1 & 134.5 & -15.4 & 213 & 186.7 & 26.3 \\
\hline $\mathrm{HFE} @ \mathrm{VCE}=-5 \mathrm{~V}, \mathrm{IC}=-5 \mathrm{~mA}$ & 111.6 & 127.1 & -15.5 & 212.7 & 140.1 & $-18,4$ \\
\hline $\mathrm{HFE} @ \mathrm{VCE}=-5 \mathrm{~V}, \mathrm{IC}=-20 \mathrm{~mA}$ & 104.7 & 118.8 & -14.1 & 113,9 & 130.8 & .16 .9 \\
\hline VBEON $(\mathrm{mV}) @ V C E=-5 \mathrm{~V}, I \mathrm{C}=.5 \mathrm{~mA}$ & 843 & 842 & 1 & 848 & 848.5 & .0 .5 \\
\hline VBEON (mV)@VCE $=-5 V, I C=-20 \mathrm{~mA}$ & 875.5 & 874.5 & 1 & 881 & 881 & 0 \\
\hline $\mathrm{HFE} \otimes \mathrm{VCE}=5 \mathrm{~V}, \mathrm{IC}=5 \mathrm{~mA}$ & 35.8 & 57.2 & $.21,4$ & 72.4 & 35.8 & 36.6 \\
\hline $\mathrm{HFE} @ V C E=S \mathrm{~V}, I \mathrm{C}=20 \mathrm{~mA}$ & 36.1 & 35.8 & 0.3 & 70.2 & 56.7 & 13.5 \\
\hline VBEON $(\mathrm{mV}) @ V C E=5 \mathrm{~V}, I \mathrm{C}=5 \mathrm{~mA}$ & 861.5 & 864 & -2.5 & 873,5 & 876 & .2 .5 \\
\hline $\mathrm{VBEON}(\mathrm{mV}) @ \mathrm{VCE}=5 \mathrm{~V}, I \mathrm{C}=20 \mathrm{~mA}$ & 907.5 & 913.5 & -6 & 931 & 928.5 & -2.5 \\
\hline $1+\mathrm{HFE} O \mathrm{VCB}=-5 \mathrm{~V}, \mathrm{IE}=-10 \mu \mathrm{A}$ & 113,9 & 99.5 & 14.4 & 173.6 & 216 & -42.4 \\
\hline $\mathrm{HFE} @ \mathrm{VCE}=-5 \mathrm{~V}, \mathrm{IC}=-5 \mathrm{~mA}$ & 107.6 & 120.2 & -12.6 & 116.1 & 132.6 & -16.5 \\
\hline $\mathrm{HFE} Q \mathrm{VCE}=-.5 \mathrm{~V}, \mathrm{IC}=-20 \mathrm{~mA}$ & 101 & 112.6 & .11 .6 & 108.7 & 123.9 & -15.2 \\
\hline VBEON $(\mathrm{mIV}) @ V C E=.5 \mathrm{~V}, I \mathrm{IC}=-5 \mathrm{~mA}$ & 843 & 842 & 1 & 847.5 & 848.5 & -1 \\
\hline VBEON $(\mathrm{mV})(\mathrm{VCE}=-5 \mathrm{~V}, \mathrm{IC}=-20 \mathrm{~mA}$ & 875.5 & 875 & 0.5 & 880 & 881 & -1 \\
\hline
\end{tabular}




\begin{tabular}{|c|c|c|c|c|c|c|}
\hline \multirow{2}{*}{$\frac{\text { TABLE A34: AVERA GE AND STANL }}{\text { TEST }}$} & \multicolumn{3}{|c|}{ AVERAGE VALUES } & \multicolumn{3}{|c|}{ STANDARD DEVIATIION } \\
\hline & Freon & Dl Water & Delta & Freon & DIWater & Delta \\
\hline$V F(m V) @ \mathbb{I F}=1 \mathrm{~mA}$ & 782.88 & 784,02 & -1.14 & 2.39 & 2.84 & $.0,45$ \\
\hline $\mathrm{VF}(\mathrm{mIV}) \otimes \mathrm{IF}=10 \mathrm{~mA}$ & 877.38 & 878.77 & $-1,39$ & 2.79 & 3.04 & 0.25 \\
\hline $1+\mathrm{HPE} Q \mathrm{VCB}=-5 \mathrm{~V}, \mathrm{IE}=-10 \mu \mathrm{A}$ & 124.39 & 140,73 & -16.34 & 4.79 & 2.14 & 2.65 \\
\hline $\mathrm{HFE} Q \mathrm{VCE}=-5 \mathrm{~V}, \mathrm{IC}=-5 \mathrm{~mA}$ & 116.69 & 132.75 & $.16,06$ & 2.65 & 2.01 & 0.64 \\
\hline HFE Q VCE $=.5 \mathrm{~V}, I \mathrm{C}=.20 \mathrm{~mA}$ & 104.38 & 117,41 & -13.03 & 3.73 & 3.81 & -0.08 \\
\hline VBEON $(\mathrm{mV}) @ V C E=-5 \mathrm{~V}, \mathrm{IC}=-5 \mathrm{~mA}$ & 845,89 & 844.81 & 1.08 & 1.31 & 1.35 & .0 .04 \\
\hline $\mathrm{VBEON}(\mathrm{mV}) @ V C E=-5 \mathrm{~V}, \mathrm{IC}=-20 \mathrm{~mA}$ & 852.7 & 851.86 & 0.84 & 24,16 & 20.01 & 4.15 \\
\hline $\mathrm{HFE} @ \mathrm{VCE}=5 \mathrm{~V}, \mathrm{IC}=5 \mathrm{~mA}$ & 43.9 & 47.83 & .3 .93 & 7.99 & 5.64 & 2.35 \\
\hline $\mathrm{HFE} \otimes \mathrm{VCE}=5 \mathrm{~V}, \mathrm{IC}=20 \mathrm{~mA}$ & 43.9 & 47.99 & 4.09 & 7.54 & 5.44 & 2.10 \\
\hline VBEON $(\mathrm{mV}) @ V C E=5 \mathrm{~V}, 1 \mathrm{C}=5 \mathrm{~mA}$ & 869.81 & 867.74 & 2.07 & 2.65 & 3.32 & $-0,67$ \\
\hline VBEON $(\mathrm{mV}) @ \mathrm{VCE}=5 \mathrm{~V}, I \mathrm{C}=20 \mathrm{~mA}$ & 922.37 & 919.2 & 3.17 & 4.79 & 4.49 & 0.3 \\
\hline $1+\mathrm{HFE} @ Q \mathrm{VCB}=.5 \mathrm{~V}, \mathrm{IE}=-10 \mu \mathrm{A}$ & 119.28 & 133.27 & -13.99 & 9.37 & 4.40 & 4.97 \\
\hline $\mathrm{HFE} @ \mathrm{VCE}=-5 \mathrm{~V}, \mathrm{IC}=-5 \mathrm{~mA}$ & 110.79 & 125.36 & -14.57 & 2.19 & 2.32 & -0.13 \\
\hline $\mathrm{HFE} @ V C E=-5 \mathrm{~V}, \mathrm{IC}=-20 \mathrm{~mA}$ & 103.85 & 117.25 & -13.4 & 1.95 & 2.12 & $-0,17$ \\
\hline VBEON $(\mathrm{mV}) @ V C E=-5 \mathrm{~V}, I \mathrm{C}=-5 \mathrm{~mA}$ & 845.9 & 844.88 & 1.02 & 1.26 & 1.42 & -0.16 \\
\hline VBEON $(\mathrm{IVV}) @ \mathrm{VCE}=-5 \mathrm{~V}, \mathrm{IC}=-20 \mathrm{~mA}$ & 878.35 & 877.19 & 1,16 & 1.15 & 1.35 & $-0,2$ \\
\hline $\mathrm{VF}(\mathrm{mV}) @ \mathrm{IF}=\operatorname{lmA}$ & 783.6 & 784.53 & -0.93 & 2.08 & 2.31 & .0 .23 \\
\hline$V F(m V) @ I F=10 \mathrm{~mA}$ & 878.77 & 879,69 & $.0,92$ & 2.38 & 2.56 & -0.18 \\
\hline $1+\mathrm{HFE} @ \mathrm{VCB}=-5 \mathrm{~V}, 1 \mathrm{E}=-10 \mu \mathrm{A}$ & 126.3 & 141.39 & -15.09 & 11.83 & 7.26 & 4.57 \\
\hline $\mathrm{HFE} @ \mathrm{VCE}=-5 \mathrm{~V}, \mathrm{IC}=-5 \mathrm{~mA}$ & 117.49 & 132.13 & .14 .64 & 2.48 & 2.35 & 0.13 \\
\hline $\mathrm{HFE} @ \mathrm{VCE}=-5 \mathrm{~V}, \mathrm{IC}=-20 \mathrm{~mA}$ & 110,03 & 123.43 & $-13,4$ & 2.25 & 2.14 & 0.11 \\
\hline VBEON (mV)@VCE $=-5 V, I C=-5 \mathrm{~mA}$ & 845.64 & 844.75 & 0.89 & 1.3 & 1.42 & -0.12 \\
\hline $\mathrm{VBEON}(\mathrm{mV}) @ \mathrm{VCE}=-.5 \mathrm{~V}, \mathrm{IC}=-20 \mathrm{~mA}$ & 878 & 877.03 & 0.97 & 1.25 & 1.34 & -0.09 \\
\hline $\mathrm{HFE} @ \mathrm{VCE}=5 \mathrm{~V}, \mathrm{IC}=5 \mathrm{~mA}$ & 55,06 & 41.67 & 13.39 & 11.12 & 5.27 & 5.85 \\
\hline $\mathrm{HFE} @ V C E=5 \mathrm{~V}, \mathrm{IC}=20 \mathrm{~mA}$ & 54.30 & 41.89 & 12.41 & 10.39 & 5.14 & 5.25 \\
\hline $\mathrm{VBEON}(\mathrm{mV}) @ \mathrm{VCE}=5 \mathrm{~V}, \mathrm{IC}=5 \mathrm{~mA}$ & 866.7 & 870,85 & -4.15 & 3.38 & 2.91 & 0.47 \\
\hline VBEON $(\mathrm{mV}) @ \mathrm{VCE}=5 \mathrm{~V}, I \mathrm{C}=20 \mathrm{~mA}$ & 916.78 & 923.12 & -6.34 & 6.29 & 3.68 & 2.61 \\
\hline $1+\mathrm{HFE} @ \mathrm{~V} \mathrm{VB}=-5 \mathrm{~V}, \mathrm{IE}=-10 \mu \mathrm{A}$ & 121.9 & 134.24 & -12.34 & 8.92 & 12.53 & -3.61 \\
\hline HFE@ VCE $=-5 \mathrm{~V}, \mathrm{IC}=-5 \mathrm{~mA}$ & 112.38 & 125.6 & -13.22 & 1.89 & 2.11 & -0.23 \\
\hline HFE @ VCE $=-5 \mathrm{~V}, I \mathrm{C}=-20 \mathrm{~mA}$ & 105.18 & 117.45 & $-12.2^{\prime \prime 7}$ & 1.68 & 1.94 & -0.26 \\
\hline VBEON $(\mathrm{mV}) @ \mathrm{VCE}=-5 \mathrm{~V}, \mathrm{IC}=-5 \mathrm{~mA}$ & 845.55 & 844.84 & 0.71 & 1.3 & 1.4 & -0.1 \\
\hline VBEON $(\mathrm{mV}) @ V C E=-5 \mathrm{~V}, I \mathrm{C}=-20 \mathrm{~mA}$ & 877.99 & 877.21 & 0.78 & 1.18 & 1.28 & -0.1 \\
\hline
\end{tabular}




\begin{tabular}{|c|c|c|c|c|c|c|}
\hline \multicolumn{7}{|c|}{ TABLE A35: MINIMUM AND MAXIMUM VALUES OF ELECTRYCAL TE } \\
\hline TEST & \multicolumn{3}{|c|}{ MINIMUM VALUES } & \multicolumn{3}{|c|}{ MAXIMUM VALUES } \\
\hline & Freon & DI Water & Delta & Freon & DI Water & Delta \\
\hline $\mathrm{IR}(\mu \mathrm{A}) @ \mathrm{VR}=55 \mathrm{~V}$ & 6.55 & 7.56 & $-1,01$ & 11.3 & 11.8 & -0.5 \\
\hline $\mathrm{VF}(\mathrm{mV}) @ I F=1 \mathrm{~mA}$ & 401.5 & 400.5 & 1 & 415.5 & 414 & 1.5 \\
\hline$V F(m V) @ I F=10 \mathrm{~mA}$ & 572 & 567 & 5 & 586 & 585.5 & 0.5 \\
\hline $\mathrm{ICBO}(\mathrm{nA}) @ \mathrm{VCB}=-50 \mathrm{~V}$ & 17 & 19 & -2 & 82 & 31 & 51 \\
\hline $\operatorname{ICER}(\mathrm{nA}) @ \mathrm{VCE}=-50 \mathrm{~V}, \mathrm{R}=1 \mathrm{~K}$ & 24 & 24 & 0 & 98 & 40 & 58 \\
\hline $\operatorname{IEBO}(\mathrm{nA}) \otimes \mathrm{VEB}=-4 \mathrm{~V}$ & 3.3 & 1.3 & 2 & 12 & 7.2 & 4.8 \\
\hline HFE@VCE $=-5 \mathrm{~V}, I \mathrm{C}=-5 \mathrm{~mA}$ & 325 & 360 & .35 & 347 & 373 & -26 \\
\hline $\mathrm{HFE} @ \mathrm{VCE}=-5 \mathrm{~V}, \mathrm{IC}=-20 \mathrm{~mA}$ & 287 & 315 & -28 & 305 & 326 & .21 \\
\hline VBEON $(\mathrm{mV}) @ \mathrm{VCE}=-5 \mathrm{~V}, I \mathrm{C}=-5 \mathrm{~mA}$ & 504.5 & 503 & 1.5 & 514 & 508 & 6 \\
\hline VBEON $(\mathrm{mV}) @ \mathrm{VCE}=-5 \mathrm{~V}, I \mathrm{C}=-20 \mathrm{~mA}$ & 562.5 & 560.5 & 2 & 571.5 & 566 & 5.5 \\
\hline$I C E R(n A) @ V C E=13 V, R=1 K$ & 1220 & 1130 & 90 & 3320 & 2820 & 500 \\
\hline $\mathrm{IEBO}(\mathrm{nA}) @ \mathrm{VEB}=4 \mathrm{~V}$ & 120 & 118 & 2 & 648 & 380 & 268 \\
\hline HFE @ VCE $=5 \mathrm{~V}, \mathrm{IC}=5 \mathrm{~mA}$ & 110.9 & 114.7 & -3.8 & 229 & 193.1 & 35.9 \\
\hline $\mathrm{HPE} @ \mathrm{VCE}=5 \mathrm{~V}, \mathrm{IC}=20 \mathrm{~mA}$ & 114.3 & 116.6 & -2.3 & 216 & 186 & 30 \\
\hline $\mathrm{VBEON}(\mathrm{mV}) @ \mathrm{VCE}=5 \mathrm{~V}, \mathrm{IC}=5 \mathrm{~mA}$ & 522.5 & 520.5 & 2 & 539.5 & 539 & 0.5 \\
\hline VBEON $(\mathrm{mV}) @ V C E=5 \mathrm{~V}, \quad I \mathrm{C}=20 \mathrm{~mA}$ & 586.5 & 587 & -0.5 & 608.5 & 607.5 & 1 \\
\hline $\mathrm{VCES}(\mathrm{mV}) @ \mathrm{IC}=10 \mathrm{~mA}, \mathrm{LB}=1 \mathrm{~mA}$ & 156 & $16 ! .5$ & -5.5 & 175.5 & 179.5 & -4 \\
\hline $\mathrm{ICBO}(\mathrm{nA}) @ \mathrm{VCB}=.50 \mathrm{~V}$ & 22 & 19 & 3 & 93 & 52 & 41 \\
\hline $\operatorname{ICER}(\mathrm{nA}) @ V C=-50 \mathrm{~V}, \mathrm{R}=1 \mathrm{~K}$ & 27 & 23 & 4 & 322 & 166 & 156 \\
\hline $\operatorname{IEBO}(n \mathrm{~A}) @ V E B=-4 V$ & 2.4 & 1.6 & 0.8 & 9.4 & 4.9 & 4.5 \\
\hline $\mathrm{HFE} @ \mathrm{VCE}=-5 \mathrm{~V}, \mathrm{IC}=-5 \mathrm{~mA}$ & 315 & 351 & .36 & 336 & 366 & -30 \\
\hline $\mathrm{HFE} @ V C E=-5 \mathrm{~V}, \mathrm{IC}=-20 \mathrm{~mA}$ & 278 & 308 & -30 & 296 & 320 & .24 \\
\hline VBEON $(\mathrm{mV}) @ \mathrm{VCE}=.5 \mathrm{~V}, \mathrm{IC}=-5 \mathrm{~mA}$ & 504.5 & 502 & 2.5 & 514 & 508.5 & 5.5 \\
\hline VBEON $(m V) @ V C E=-5 V, I C=-20 m A$ & 563 & 560.5 & 2.5 & 571.5 & 566.5 & 5 \\
\hline $\mathrm{IR}(\mu \mathrm{A}) @ \mathrm{VR}=55 \mathrm{~V}$ & 6.2 & 7.41 & $-1,21$ & 24.1 & 11.8 & 12.3 \\
\hline$V F(m V) @ I F=1 m A$ & 407 & 404.5 & 2.5 & 417.5 & 414 & 3,5 \\
\hline $\mathrm{VF}(\mathrm{mV}) @ \mathrm{IF}=10 \mathrm{~mA}$ & 577.5 & 572.5 & 5 & 588.5 & 586 & 2.5 \\
\hline$I C B O(n A) @ V C B=-50 V$ & 20 & 18 & 2 & 57 & 30 & 27 \\
\hline $\mathrm{ICER}(\mathrm{nA}) @ \mathrm{VCE}=-50 \mathrm{~V}, \mathrm{R}=1 \mathrm{~K}$ & 27 & 22 & 5 & 101 & 41 & 60 \\
\hline IEBO $(\mathrm{nA}) @ \mathrm{VEB}=-4 \mathrm{~V}$ & 3.6 & 2 & 1.6 & 14 & 7.2 & 6.8 \\
\hline $\mathrm{HFE} @ \mathrm{VCE}=-5 \mathrm{~V}, \mathrm{IC}=-5 \mathrm{~mA}$ & 324 & 359 & .35 & 348 & 375 & -27 \\
\hline $\mathrm{HFE} @ \mathrm{VCE}=-5 \mathrm{~V}, \mathrm{IC}=-20 \mathrm{~mA}$ & 286 & 315 & .29 & 305 & 328 & -23 \\
\hline VBEON $(\mathrm{mV}) @ \mathrm{VCE}=-5 \mathrm{~V}, \mathrm{IC}=-5 \mathrm{~mA}$ & 504.5 & 502 & 2.5 & 515 & 508.5 & 6.5 \\
\hline VBEON $(m \mathrm{~m}) @ V C E=-5 \mathrm{~V}, \mathrm{IC}=-20 \mathrm{~mA}$ & 562.5 & 560.5 & 2 & 572 & 566.5 & 5.5 \\
\hline $\operatorname{ICER}(\mathrm{nA}) @ \mathrm{VCE}=13 \mathrm{~V}, \mathrm{R}=1 \mathrm{~K}$ & 832 & 1130 & -298 & 1580 & 1510 & 70 \\
\hline IEBO (nA)@VEB $=4 \mathrm{~V}$ & 112 & 117 & -5 & 536 & -373 & 163 \\
\hline $\mathrm{HFE} @ \mathrm{VCE}=5 \mathrm{~V}, \mathrm{IC}=5 \mathrm{~mA}$ & 116.8 & 117.9 & -1.1 & 269 & 199.6 & 69.4 \\
\hline HFE @ VCE $=5 \mathrm{~V}, \mathrm{IC}=20 \mathrm{~mA}$ & 119.2 & 120 & -0.8 & 247 & 193.1 & 53.9 \\
\hline $\mathrm{VBEON}(\mathrm{mV}) @ \mathrm{VCE}=5 \mathrm{~V}, \mathrm{IC}=5 \mathrm{~mA}$ & 518 & 521.5 & -3.5 & 538 & 538.5 & -0.5 \\
\hline VBEON $(\mathrm{mV}) @ \mathrm{VCE}=5 \mathrm{~V}, \quad \mathrm{IC}=20 \mathrm{~mA}$ & 581.5 & 587 & -5.5 & 606.5 & 607.5 & -1 \\
\hline VCES $(\mathrm{mV}) @ I C=10 \mathrm{~mA}, \mathrm{IB}=1 \mathrm{~mA}$ & 152 & 161.5 & -9.5 & 175.5 & 179 & -3.5 \\
\hline $\mathrm{ICBO}(\mathrm{nA}) @ \mathrm{VCB}=-50 \mathrm{~V}$ & 23 & 18 & 5 & 61 & 35 & 26 \\
\hline $\mathrm{ICER}(\mathrm{nA}) @ \mathrm{VC}=-50 \mathrm{~V}, \mathrm{R}=1 \mathrm{~K}$ & 30 & 23 & 7 & 78 & 46 & 32 \\
\hline $\mathrm{IEBO}(\mathrm{nA}) @ \mathrm{VEB}=-4 \mathrm{~V}$ & 3.6 & 1.5 & 2.1 & 10 & 6.4 & 3.6 \\
\hline HFE @VCE $=-5 \mathrm{~V}, \mathrm{IC}=-5 \mathrm{~mA}$ & 316 & 352 & -36 & 337 & 371 & -34 \\
\hline $\mathrm{HFE} @ \mathrm{VCE}=-5 \mathrm{~V}, \mathrm{IC}=-20 \mathrm{~mA}$ & 279 & 309 & -30 & 295 & 324 & .29 \\
\hline VBEON $(\mathrm{mV}) @ \mathrm{VCE}=-5 \mathrm{~V}, \mathrm{IC}=-5 \mathrm{~mA}$ & 504.5 & 502 & 2.5 & 512.5 & 508.5 & 4 \\
\hline VBEON $(m \mathrm{~V}) @ \mathrm{VCE}=-5 \mathrm{~V}, \mathrm{IC}=-20 \mathrm{~mA}$ & 562.5 & 560.5 & 2 & 570.5 & 566.5 & 4 \\
\hline
\end{tabular}


TABLE A36: AVERAGE AND STANDARD DEVIATION OF ELECTRICAL TEST DATA FOR M-50 AT $100^{\circ} \mathrm{C}$

\begin{tabular}{|c|c|c|c|c|c|c|}
\hline \multirow[t]{2}{*}{ TEST } & \multicolumn{3}{|c|}{ AVERAGE VALUES } & \multicolumn{3}{|c|}{ STA VDARD DEVIATION } \\
\hline & Freon & DI Water & Delta & Freon & DI Water & Delta \\
\hline $\mathrm{IR}(\mu \mathrm{A}) @ \mathrm{VR}=55 \mathrm{~V}$ & 9.02 & 10.1 & -1.08 & 0.993 & 1.01 & -0.017 \\
\hline$V F(m V) @ I F=1 m A$ & 410.48 & 409.98 & 0.5 & 2.48 & 2.75 & -0.27 \\
\hline $\mathrm{VF}(\mathrm{mV}) @ \mathrm{IF}=10 \mathrm{~mA}$ & 580.23 & 580.94 & -0.71 & 2.99 & 3.5 & -0.51 \\
\hline $\mathrm{ICBO}(\mathrm{nA}) @ \mathrm{VCB}=-50 \mathrm{~V}$ & 30 & 23 & 7 & 9.5 & 2.4 & 7.1 \\
\hline $\operatorname{ICER}(\mathrm{nA}) @ \mathrm{VCE}=-50 \mathrm{~V}, \mathrm{R}=1 \mathrm{~K}$ & 40 & 28 & 12 & 13 & 2.9 & 10.1 \\
\hline IEBO (nA)@ VEB $=-4 \mathrm{~V}$ & 5.5 & 3.8 & 1.7 & 1.6 & 0.72 & 0.88 \\
\hline $\mathrm{HFE} @ \mathrm{VCE}=-5 \mathrm{~V}, \mathrm{IC}=-5 \mathrm{~mA}$ & 335.37 & 368.69 & -33.32 & 6.75 & 2.81 & 3.94 \\
\hline HFE @ VCE $=-5 \mathrm{~V}, \mathrm{IC}=-20 \mathrm{~mA}$ & 295.28 & 322.54 & -27.26 & 5.49 & 2.15 & 3.34 \\
\hline VBEON $(\mathrm{mV}) @ \mathrm{VCE}=-5 \mathrm{~V}, \mathrm{IC}=-5 \mathrm{~mA}$ & 508.77 & 506.48 & 2.29 & 1.75 & 1.39 & 0.36 \\
\hline VBEON $(\mathrm{mV}) @ \mathrm{VCE}=-5 \mathrm{~V}, \mathrm{IC}=-20 \mathrm{~mA}$ & 566.43 & 564.16 & 2.27 & 1.62 & 1.22 & 0.4 \\
\hline $\operatorname{ICER}(n A) @ V C E=13 \mathrm{~V}, \mathrm{R}=1 \mathrm{~K}$ & 1500 & 1380 & 120 & 257 & 279 & -22 \\
\hline IEBO (nA)@ VEB =4V & 175 & 143 & 32 & 85 & 43 & 42 \\
\hline HFE @ VCE $=5 \mathrm{~V}, \mathrm{IC}=5 \mathrm{~mA}$ & 147.64 & 159.85 & -12.21 & 32.92 & 20.76 & 12.16 \\
\hline $\mathrm{HFE} @ \mathrm{VCE}=5 \mathrm{~V}, \mathrm{IC}=20 \mathrm{~mA}$ & 146.2 & 158.59 & -12.39 & 28.56 & 18.55 & 10.01 \\
\hline $\mathrm{VBEON}(\mathrm{mV}) @ \mathrm{VCE}=5 \mathrm{~V}, \mathrm{IC}=5 \mathrm{~mA}$ & 531.78 & 527.63 & 4.15 & 3.49 & 4.51 & -1.02 \\
\hline VBEON $(\mathrm{mV}) @ \mathrm{VCE}=5 \mathrm{~V}, I \mathrm{IC}=20 \mathrm{~mA}$ & 599.28 & 594.27 & 5.01 & 5.22 & 5.23 & -0.01 \\
\hline $\operatorname{VCES}(\mathrm{mV}) @ \mathrm{IC}=10 \mathrm{~mA}, \mathrm{IB}=1 \mathrm{~mA}$ & 168.28 & 166.55 & 1.73 & 5.07 & 5.1 & -0.03 \\
\hline $\mathrm{ICBO}(\mathrm{nA}) @ \mathrm{VCB}=-50 \mathrm{~V}$ & 36 & 23 & 13 & 11 & 4.6 & 6.4 \\
\hline $\operatorname{ICER}(\mathrm{nA}) @ \mathrm{VC}=-50 \mathrm{~V}, \mathrm{R}=1 \mathrm{~K}$ & 52. & 33 & 19 & 42 & 21 & 21 \\
\hline IEBO (nA)@VEB=-4V & 6 & 3.8 & 2.2 & 1.5 & 0.71 & 0.79 \\
\hline $\mathrm{HFE} @ \mathrm{VCE}=-5 \mathrm{~V}, \mathrm{IC}=-5 \mathrm{~mA}$ & 324.42 & 358.33 & -33.91 & 6.28 & 3.97 & 2.31 \\
\hline HFE @ VCE $=-5 \mathrm{~V}, \mathrm{IC}=-20 \mathrm{~mA}$ & 286.08 & 313.81 & -27.73 & 4.99 & 3.17 & 1.82 \\
\hline $\mathrm{VBEON}(\mathrm{mV}) @ \mathrm{VCE}=-5 \mathrm{~V}, \mathrm{IC}=-5 \mathrm{~mA}$ & 508.84 & 506.45 & 2.39 & 1.73 & 1.43 & 0.3 \\
\hline VBEON $(m V) @ V C E=-5 V, I C=-20 m A$ & 566.45 & 564.2 & 2.2 .5 & 1.61 & 1.22 & 0.39 \\
\hline $\operatorname{IR}(\mu \mathrm{A}) @ \mathrm{VR}=55 \mathrm{~V}$ & 9.55 & 9.5 & 0.05 & 2.59 & 1.03 & 1.56 \\
\hline $\mathrm{VF}(\mathrm{mV}) @ \mathrm{IF}=1 \mathrm{~mA}$ & 410.84 & 411.53 & -0.69 & 1.97 & 2.1 & -0.13 \\
\hline $\mathrm{VF}(\mathrm{mV}) @ \mathrm{IF}=10 \mathrm{~mA}$ & 581.73 & 582.63 & -0.9 & 2.23 & 2.42 & -0.19 \\
\hline$I C B O(n A) @ V C B=-50 V$ & 31 & 23 & 8 & 7.3 & 2.2 & 5.1 \\
\hline $\mathrm{ICER}(\mathrm{nA}) @ \mathrm{VCE}=-50 \mathrm{~V}, \mathrm{R}=1 \mathrm{~K}$ & 42 & 28 & 14 & 12 & 3.6 & 8.4 \\
\hline IEBO (nA)@VEB $=-4 \mathrm{~V}$ & 6 & 4 & 2 & 1.7 & 0.74 & 0.96 \\
\hline $\mathrm{HFE}, \mathrm{V} \mathrm{CE}=-5 \mathrm{~V}, \mathrm{IC}=-5 \mathrm{~mA}$ & 338.3 & 367.89 & -29.59 & 6.37 & 3.12 & 3.25 \\
\hline $\mathrm{HFE} @ \mathrm{VCE}=-5 \mathrm{~V}, \mathrm{IC}=-20 \mathrm{~mA}$ & 297.77 & 322 & -24.23 & 5.17 & 2.52 & 2.65 \\
\hline VBEON $(\mathrm{mV}) @ \mathrm{VCE}=-5 \mathrm{~V}, \mathrm{IC}=-5 \mathrm{~mA}$ & 508.39 & 506.54 & 1.85 & 1.71 & 1.5 & 0.21 \\
\hline VBEON $(m V) @ V C E=-5 V, I C=-20 m A$ & 566.09 & 564.19 & 1.9 & 1.63 & 1.19 & 0.41 \\
\hline $\operatorname{ICER}(\mathrm{nA}) @ \mathrm{VCE}=13 \mathrm{~V}, \mathrm{R}=1 \mathrm{~K}$ & 1340 & 1320 & 20 & 211 & 82 & 129 \\
\hline IEBO (nA) @ VEB $=4 \mathrm{~V}$ & 185 & 161 & 24 & 87 & 52 & 35 \\
\hline HFE @ VCE $=5 \mathrm{~V}, I \mathrm{C}=5 \mathrm{~mA}$ & 194.37 & 137.55 & 56.82 & 46.34 & 20.01 & 26.33 \\
\hline HFE @ VC $\mathrm{B}=5 \mathrm{~V}, \mathrm{IC}=20 \mathrm{~mA}$ & 185.97 & 137.9 & 48.07 & 39.35 & 17.98 & 21.37 \\
\hline VBEON $(\mathrm{mV}) @ \mathrm{VCE}=5 \mathrm{~V}, \mathrm{IC}=5 \mathrm{~mA}$ & 527.72 & 533.13 & -5.41 & 4.8 & 4.46 & 0.34 \\
\hline VBEON $(\mathrm{mV}) @ \mathrm{VCE}=5 \mathrm{~V}, \mathrm{IC}=20 \mathrm{~mA}$ & 593.38 & 600.73 & -7.35 & 6.8 & 5.14 & 1.66 \\
\hline $\mathrm{VCES}(\mathrm{mV}) @ \mathrm{IC}=10 \mathrm{~mA}, \mathrm{IB}=1 \mathrm{~mA}$ & 159.39 & 173.15 & -13.76 & 5.2 & 4.75 & 0.45 \\
\hline $\mathrm{ICBO}(\mathrm{nA}) @ \mathrm{VCB}=-50 \mathrm{~V}$ & 38 & 23 & 15 & 7.4 & 3 & 4.4 \\
\hline $\operatorname{ICER}(\mathrm{nA}) @ V C=-50 \mathrm{~V}, \mathrm{R}=1 \mathrm{~K}$ & 50 & 29 & 21 & 10 & 3.8 & 6.2 \\
\hline IEBO (nA)@VEB $=-4 \mathrm{~V}$ & 6.7 & 3.9 & 2.8 & 1.5 & 1.1 & 0.4 \\
\hline $\mathrm{HFE} @ \mathrm{VCE}=-5 \mathrm{~V}, \mathrm{IC}=-5 \mathrm{~mA}$ & 329.82 & 358.74 & -28.92 & 4.88 & 3.34 & 1.54 \\
\hline HFE @ VCE $=-5 \mathrm{~V}, \mathrm{IC}=-20 \mathrm{~mA}$ & 290.17 & 314.11 & .23 .94 & 3.86 & 2.74 & 1.12 \\
\hline $\mathrm{VBEON}(\mathrm{mV}) @ \mathrm{VCE}=-5 \mathrm{~V}, \mathrm{IC}=-5 \mathrm{~mA}$ & 508.18 & 506.48 & 1.7 & 1.6 & 1.4 & 0.2 \\
\hline VBEON $(m V) @ V C E=-5 V, I C=-20 m A$ & 565.97 & 564.34 & 1.63 & 1.51 & 1.19 & 0.32 \\
\hline
\end{tabular}



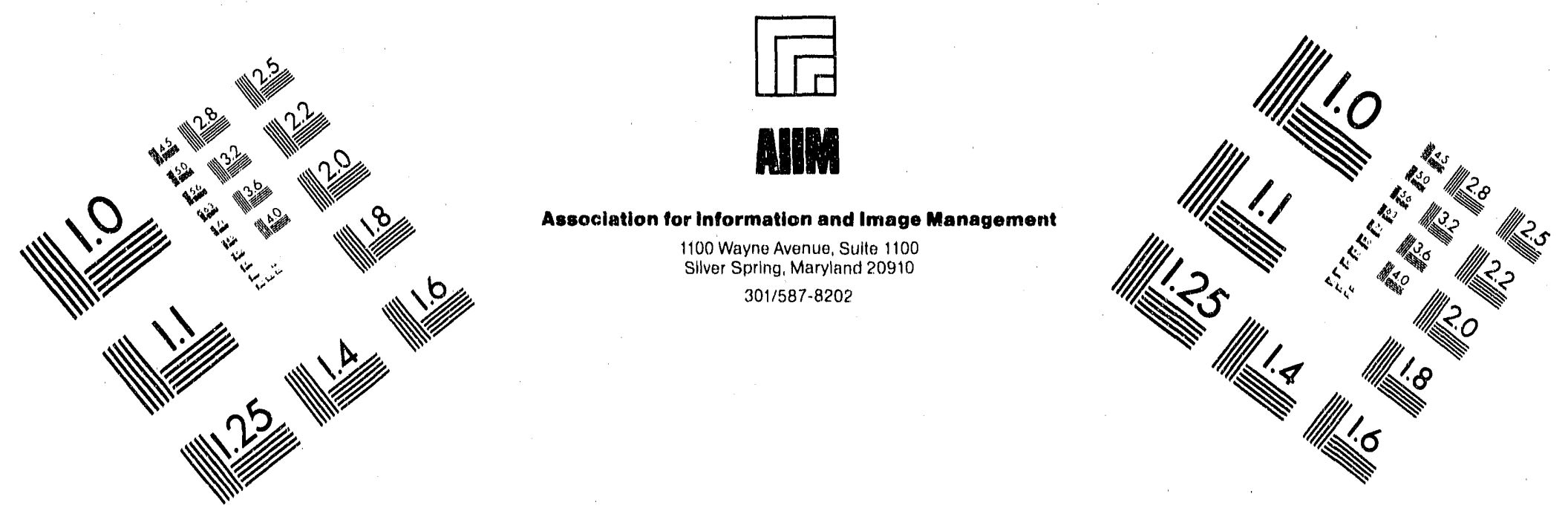

\section{Centimeter}

$\begin{array}{llllllllllllllll}1 & 2 & 3 & 4 & 5 & 6 & 7 & 8 & 9 & 10 & 11 & 12 & 13 & 14 & 15 & \mathrm{~mm}\end{array}$

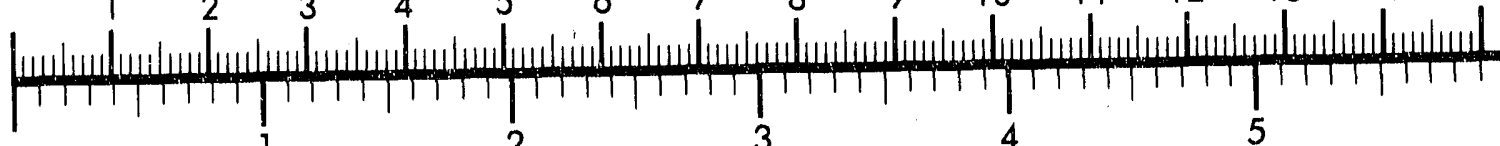

Inches
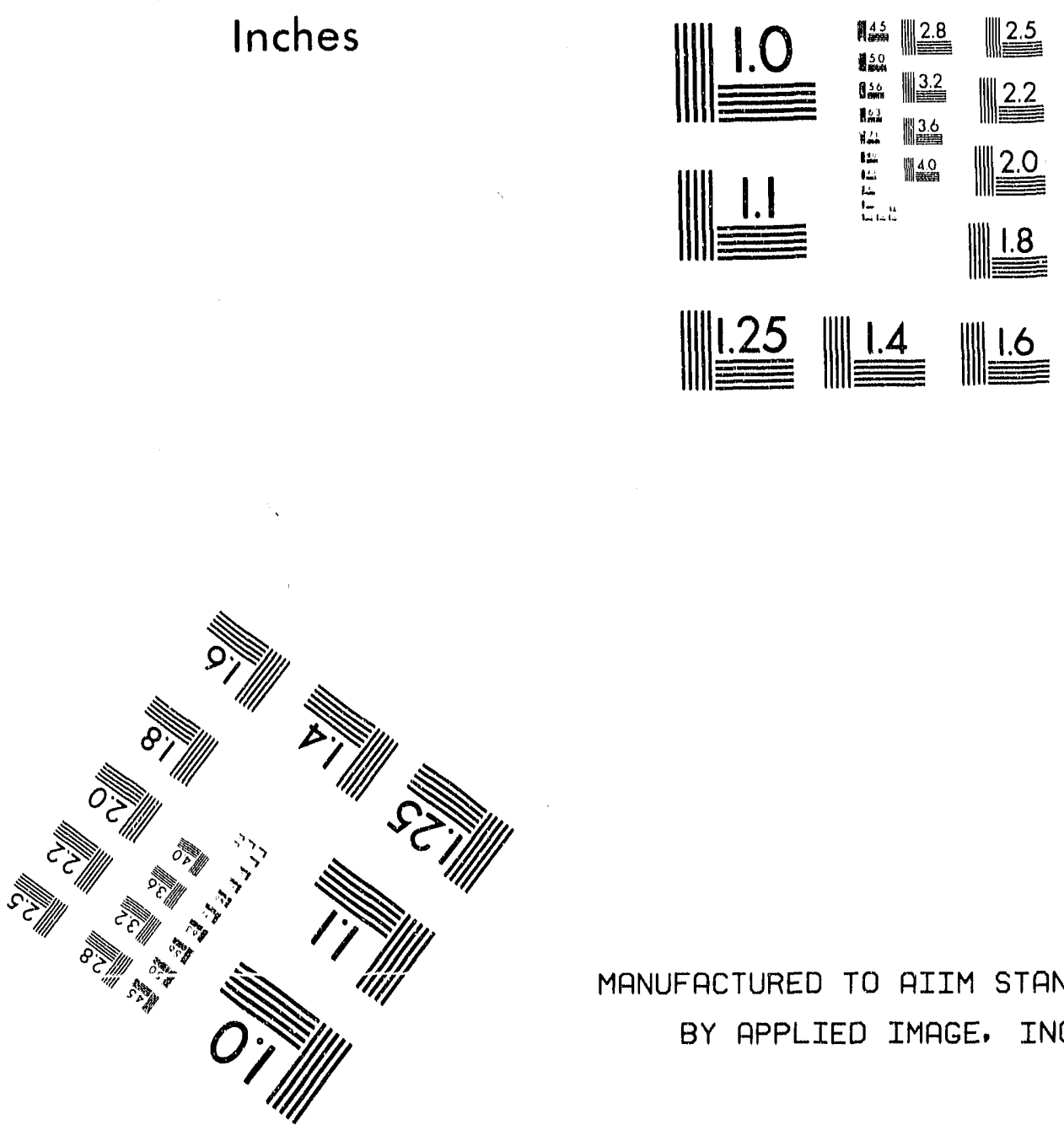

MANUFACTURED TO AIIM STANDARDS

BY APPLIED IMAGE, INC.

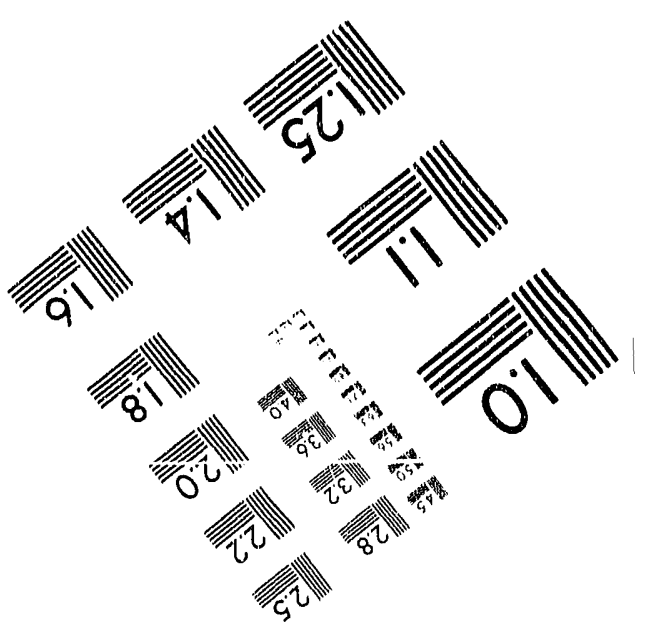


=

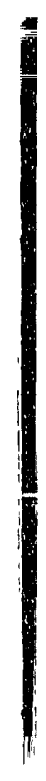



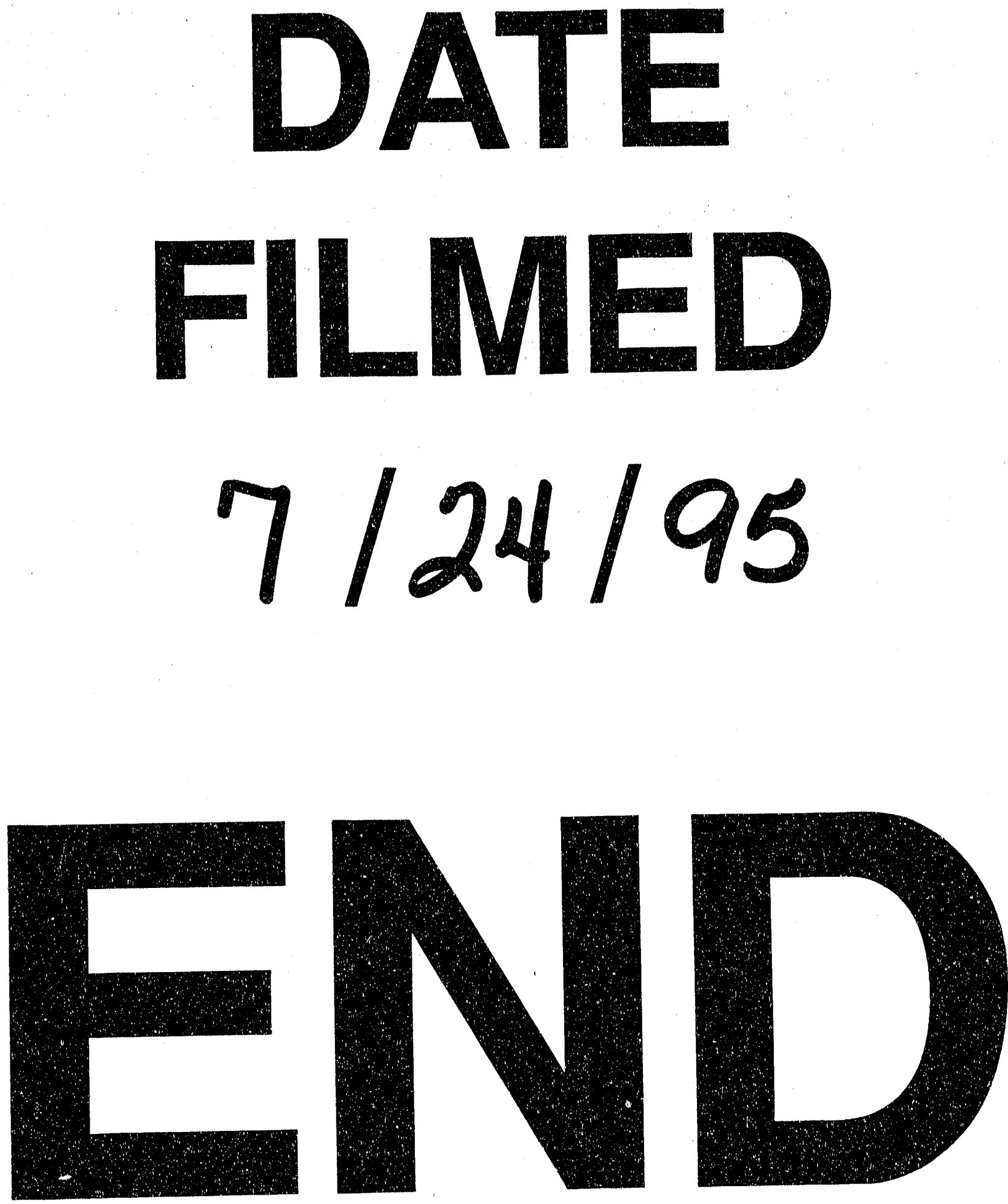